\title{
Differences in transcriptional responses to acute and chronic dietary interventions with fatty acids
}

\author{
Juri C. Matualatupauw
}




\section{Thesis committee}

\section{Promotor}

Prof. Dr A.H. Kersten

Professor of Nutrition, Metabolism and Genomics

Wageningen University \& Research

\section{Co-promotors}

Dr L.A. Afman

Assistant professor, Division of Human Nutrition

Wageningen University \& Research

Dr J. Bouwman

Senior Scientist Systems Biology

TNO Quality of life, Zeist

\section{Other members}

Prof. Dr E.J.M. Feskens, Wageningen University \& Research

Dr P. van Baarlen, Wageningen University \& Research

Prof. Dr H. Pijl, Leiden University Medical Center

Prof. Dr C.T.A. Evelo, Maastricht University

This research was conducted under the auspices of the Graduate School VLAG (Advanced studies in Food Technology, Agrobiotechnology, Nutrition and Health Sciences). 


\title{
Differences in transcriptional responses to acute and chronic dietary interventions with fatty acids
}

\author{
Juri C. Matualatupauw
}

Thesis

submitted in fulfilment of the requirements for the degree of doctor

at Wageningen University

by the authority of the Rector Magnificus,

Prof. Dr A.P.J. Mol,

in the presence of the

Thesis Committee appointed by the Academic Board

to be defended in public

on Tuesday 6 June 2017

at 1:30 p.m. in the Aula. 
Juri C. Matualatupauw

Differences in transcriptional responses to acute and chronic dietary interventions with fatty acids,

176 pages.

PhD thesis, Wageningen University, Wageningen, the Netherlands (2017)

With references, with summary in English

ISBN: 978-94-6343-207-8

DOI: $10.18174 / 414660$ 


\section{Contents}

$\begin{array}{lll}\text { Chapter } 1 \text { General introduction } & 7\end{array}$

Chapter 2 The use of transcriptomics as a tool to identify differences in the response to diet

Chapter 3 Apolipoprotein E genotype status affects habitual human blood mononuclear cell gene expression and its response to fish oil intervention

Chapter 4 Dietary medium-chain saturated fatty acids induce gene expression of energy metabolism-related pathways in adipose tissue of abdominally obese subjects

Chapter 5 Added value of using high-fat challenges to determine a high-fat high-calorie diet-induced shift towards the metabolic syndrome as measured in blood cell transcriptome

Chapter 6 Meta-analysis of high-fat challenge-induced changes in blood cell whole genome gene expression

Chapter 7 General discussion

Summary 


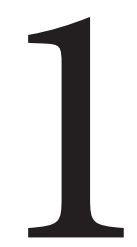

General introduction 


\section{Nutrition-related chronic disease}

Non-communicable disease, such as obesity, diabetes and cardiovascular disease were the cause of 38 million deaths in 2012, which is $68 \%$ of the total global deaths (1). The main modifiable metabolic risk factors for these diseases are high blood pressure, overweight/ obesity, raised blood glucose and raised blood lipids (1). All these risk factors have been shown to be sensitive to dietary changes both in beneficial as well as detrimental ways (2). One of the components of the diet that is thought to play a large part in the progression of these non-communicable diseases is dietary fat. Changes in dietary fat intake may help in the prevention of these diseases. Recommendations of the World Health Organization are to reduce intake of saturated fatty acids by replacing them with unsaturated fatty acids, replace transfats with unsaturated fats, and to limit excess total calorie intake (3).

\section{Types of fatty acids}

Dietary fats can have beneficial or detrimental health effects in relation to metabolic diseases such as diabetes and cardiovascular disease. In this respect, it is not the total amount of fat as a percent of energy that is found to be important, but it is the specific types of dietary fatty acids that matter most in the pathophysiology of type 2 diabetes and cardiovascular disease $(4,5)$.

Fatty acids all have the same basic structure, consisting of a chain hydrogenated carbon atoms with an acid group on one end and a methyl group on the other. Nevertheless, different types of fatty acids have been shown to have widely varying beneficial or detrimental effects on human health. These different types of fatty acids can be distinguished based on two biochemical characteristics of the carbon chain, which are 1.) the carbon chain length and 2.) the degree of saturation.

\section{Carbon chain length}

Most fatty acids contain an even number of carbon atoms in their chain, ranging from 4 to 28. They can be divided into long-chain, medium-chain and short-chain fatty acids. Longchain fatty acids have 13 or more carbon atoms in their chain. They are the most abundant type of fatty acids in food. The most common dietary long-chain fatty acids are 16 and 18 carbon atoms long. These include the saturated fatty acids palmitic (C16:0) and stearic acid (C18:0) and the monounsaturated palmitoleic acid (C16:1) and oleic acid (C18:1). Dietary polyunsaturated fatty acids include the essential n-3 fatty acid $\alpha$-linolenic acid (C18:3) and n-6 
fatty acid linoleic acid (C18:2), as well as eicosapentaenoic acid (C20:5) and docosahexaenoic acid (C22:6).

Medium-chain fatty acids have 6-12 carbon atoms in their chain. The main dietary sources of these fatty acids are coconut oil, palm oil and dairy (6). Replacement of long-chain triglycerides with medium-chain triglycerides in the diet has been shown to lead to improvements in body weight and body composition $(7,8)$.

Short-chain fatty acids (SCFAs) have fewer than 6 carbon atoms in their chain. For humans, the major source of SCFAs are the gut microbiota, which produce mainly acetate (C2:0), propionate $(\mathrm{C} 3: 0)$ and butyrate $(\mathrm{C} 4: 0)$ through the fermentation of dietary fibers. These SCFAs have been shown to affect human and mouse immune cells at the molecular level, specifically by having anti-inflammatory effects (9), though there is also evidence for proinflammatory action of SCFAs under some conditions (9). Furthermore, SCFAs produced by the gut microbiota are implicated in the progression of several diseases, including metabolic disease (10-12).

\section{Degree of saturation}

Saturated fatty acids (SFAs) have a carbon chain in which all carbon atoms are connected by single bonds. Reducing intake of dietary saturated fat was shown to reduce the risk of cardiovascular events in a meta-analysis of 15 large randomized clinical trials (13). This finding is controversial, as not all meta-analyses show this effect (14). However, replacement of saturated fat with polyunsaturated fat in the diet has been shown to reduce the risk of cardiovascular disease (15). Not all saturated fat is considered potentially detrimental, as medium-chain saturated fatty acids have been shown to have beneficial effects on body weight and body composition $(7,8)$.

Monounsaturated fatty acids (MUFAs) lack 2 hydrogen atoms in their carbon chain, which is caused by one double bond between 2 carbon atoms. Foods containing high amounts of MUFA are mostly plant-derived and include olive oil, high oleic sunflower oil and nuts. The number of studies that have examined the health effects of MUFAs is much lower than that of PUFAs. Nevertheless, strong evidence exists that replacing SFA and carbohydrates with MUFA improves various cardiovascular risk factors, as several meta-analyses and systematic reviews show that high-MUFA diets lead to increases in plasma high density lipoprotein (HDL)-cholesterol and reductions in plasma triglycerides and systolic and diastolic blood pressure (16). 
Polyunsaturated fatty acids (PUFAs) have two or more double bonds. PUFAs are found in seeds, nuts, vegetable oils and fatty fish. In general, the human intake of PUFAs is lower than that of SFAs and MUFAs in the diet. A meta-analysis of 11 European and American cohort studies found that dietary intake of PUFAs was between $2.2 \%$ and $9.0 \%$ of total energy intake, whereas for SFAs (between 9.4\% and 21.3\%) and MUFAs (between 10.2\% and 16.5\%) this was much higher (15). Nevertheless, PUFAs are found to be relatively important for human health, as increasing energy intake of PUFA in exchange for SFA was found to decrease the risk of coronary events and coronary death (15). Of special interest are the omega-3 fatty acids EPA and DHA, which are mainly found in fatty fish. Beneficial health effects of these fatty acids have been described, such as anti-inflammatory effects, decreased risk of coronary events and improved cognitive functioning in patients with mild Alzheimer's disease (17).

\section{Fatty acid metabolism}

Most fatty acids do not occur in their free form in foods or in the body. They are incorporated in triglycerides, which consist of three fatty acids bound to one glycerol molecule. Triglycerides from food are hydrolyzed in the small intestine, leading to the release of individual fatty acids. Small- and medium-chain fatty acids pass the external membrane of intestinal cells and are taken up directly into the bloodstream and are transported via the portal vein to the liver. Long-chain fatty acids are repacked into triglycerides in intestinal cells and travel via the lymphatic system to the circulation in chylomicron particles. Besides chylomicrons, several other lipoproteins are present in the circulation and play a major role in the transport of triglycerides. These lipoproteins consist of an interior of triglycerides and cholesterol, and an exterior phospholipid membrane with embedded apolipoproteins. These apolipoproteins are a structural part of lipoproteins and can also act as a cofactor or by binding to receptors on the surface of cells. Apolipoprotein C2, for example, is found on chylomicrons and VLDL and is a cofactor for lipoprotein lipase, which is the enzyme that is responsible for the hydrolysis of triglycerides (18). Apolipoprotein E (APOE) is found on several lipoproteins including VLDL, HDL and chylomicrons (19). It functions by binding receptors of the low density lipoprotein (LDL) receptor family on cells (20), and, consequently, plays a large role in cholesterol and lipid homeostasis. Of this apolipoprotein, three major isoforms exist: APOE2, APOE3 and APOE4, which have allele frequencies of $6 \%, 15 \%$ and $78 \%$ respectively (21). APOE4 is most commonly known for its relation with Alzheimer's disease, as APOE4 is the most important genetic risk factor for this disease $(22,23)$. However, APOE has been shown to be associated 
with cardiovascular disease as well, as carriers of APOE4 have a modestly increased risk of coronary heart disease $(24,25)$ and stroke $(26)$ compared with individuals with the APOE3/ APOE3 genotype.

\section{Transcriptomics}

Transcriptomics is the study of the transcriptome, which is the full set of messenger RNAs produced by a cell, cells or tissue. Frequently used methods in transcriptomics are two high-throughput techniques, microarrays and RNA-seq. For microarray analysis RNA is reverse transcribed to cDNA. Microarrays work by hybridization of this cDNA to probes on microarray-chips. As a consequence, a requirement of microarrays is that gene sequences are known. On the other hand, RNA-seq is a much newer technique and relies on sequencing of the entire RNA-sequence. As a consequence, it doesn't require gene-specific probes and can be used to detect novel transcripts or single nucleotide polymorphisms. Furthermore, RNA-SEQ has a higher dynamic range, as it does not suffer from background noise or signal saturation. However, using microarrays over RNA-sequencing has several advantages as well. Firstly, the financial costs of microarrays are much lower than RNA-seq. Moreover, microarrays have been used for several years and proven to be both sensitive and reliable. Lastly, the bioinformatics pipelines for the analysis of microarrays have been well established and tools for microarray analysis are quite user-friendly, even for non-bioinformaticians.

\section{Transcriptomics in nutrition research}

Part of the health effects of fatty acids are thought to be derived from the ability of fatty acids to regulate gene expression. The peroxisome proliferator-activated receptors (PPARs) are the best recognized sensor system for fatty acids (27). Several PPAR subtypes exist that are all able to bind to fatty acids with a special preference for PUFAs. Saturated fatty acids may promote inflammation by direct activation of toll-like receptor 4 . Other fatty acid sensors mediating gene expression regulation include SREBP1, HNF4a, NRF2, and several G-protein coupled receptors.

A drawback of the application of transcriptomics in human dietary intervention studies is the availability of tissue samples. The invasiveness of tissue biopsies makes it hard to get samples from most tissues, as the required tissue biopsies may be too much of a burden on the relatively healthy individuals that are usually participating in these studies. Tissue samples 
can therefore only be taken from easily accessible tissues. Therefore, muscle, intestine, subcutaneous adipose tissue and blood are the only feasible sources of cells to use for transcriptomics in dietary intervention studies. Blood-based cell sources, such as whole blood or subpopulations or peripheral blood mononuclear cells (PBMCs) are interesting to study in nutritional interventions studies, as they are constantly exposed to food-derived metabolites as well as endocrine signals from peripheral tissues. Microarray analyses in PBMC and whole blood have been used to characterize the molecular effects of nutritional interventions, including several with dietary fatty acids. For example, Bouwens et al. (28) studied the effects of 6 months fish-oil supplementation on whole genome gene expression profiles in PBMCs. In this study, fish-oil supplementation was found to alter gene expression in the direction of a more anti-inflammatory and anti-atherogenic profile. Another study also examined effects of fish-oil supplementation on PBMC gene expression and found increased expression of genes related to cell cycle, ER stress and apoptosis after a 7-week supplementation period (29). Van Dijk et al. (30) examined the effects of a Mediterranean diet and the replacement of SFA with MUFA in a Western-type diet for 8 weeks on PBMC whole genome gene expression. They found a decreased expression of oxidative phosphorylation genes with the Mediterranean and MUFA diets compared to the SFA diet. Furthermore, compared to the MED and SFA diets, the MUFA diet changed the expression of genes involved in B-cell receptor signaling and endocytosis signaling.

It is increasingly recognized that the adipose may play a large role in the pathogenesis of chronic non-communicable diseases, such as obesity, diabetes and cardiovascular disease (31). Adipose tissue is not only a lipid-storage tissue, but also influences the immune system and is a significant source of inflammatory signals $(31,32)$. Macrophage infiltration and an increase in pro-inflammatory signaling in the adipose tissue may be a first step in the process leading to metabolic disease (33). In support of this, in the previously mentioned study by van Dijk et al. (34), the SFA diet was found to cause an increase in pro-inflammatory gene expression compared to the MUFA diet, which caused a more anti-inflammatory gene expression profile in the adipose tissue. These altered gene expression profiles were found in the absence of changes in adipose tissue morphology or insulin sensitivity, indicating that they may be one of the first hallmarks of the nutritional effects of saturated fats and the possible subsequent metabolic disease.

Besides long-term dietary interventions, microarrays were also used to studies the acute response to dietary fatty acids. In a cross-over study by Esser et al. (35) 17 lean and 15 
obese men (50-70 years) received two $95 \mathrm{~g}$ fat shakes, high in SFAs or MUFAs. PBMC geneexpression profiles were assessed fasted and 4 hours post-prandially. High-SFA decreased expression of cholesterol biosynthesis and cholesterol uptake genes and increased expression of cholesterol efflux genes. MUFA increased inflammatory genes and PPAR- $\alpha$ targets involved in $\beta$-oxidation. Another cross-over study (36) compared the response to a PUFA and a SFA shake in young healthy men. They found that PUFA intake decreased the expression of genes in liver X receptor signaling, whereas SFA intake increased the expression of these genes. Furthermore, PUFA intake increased the expression of genes related to cellular stress responses. Matone et al. (37) studied the response to a MUFA challenge and observed an increase in expression of inflammation-related genes after 6 hours.

\section{Challenge tests}

Relatively new in nutrition research is the measurement of an individual's capacity to adapt to dietary challenges, which is called 'phenotypic flexibility' (38). Dietary challenges, such as oral glucose tolerance tests, mixed meal challenges and the previously mentioned high-fat challenges are used to examine the adaptation capacity of individuals. It is thought that using this approach may reveal subtle differences in health status that may not be detectable using conventional biomarkers of health measured in a fasted state. Combining challenge tests with -omics techniques, such as transcriptomics may enlarge differences in health status, as was observed in the previously mentioned study by Esser et al. (35). In this study, 294 genes were differentially expressed between lean and obese subjects in the fasted state. However, when comparing the response to the high-fat challenge between lean and obese subjects, 607 genes were differentially expressed upon the SFA and 2516 genes upon the MUFA challenge. These results indicate that high-fat challenges magnify differences in health, based on gene expression changes. Furthermore, several studies have shown that using transcriptome analyses to characterize the response to a high-fat challenge can provide insight into mechanisms that are involved in coping with nutrition (35-37).

\section{Individual differences in dietary responses}

Differences between individuals in the whole genome gene expression response to dietary interventions have been described. For example, the study by Esser et al. (35) examined differences between lean and obese individuals and the study by Matone et al. (37) examined 
the effect of BMI on the response to dietary challenges. Several other characteristics that may influence the transcriptomics response to dietary interventions have been studied. These include gender $(39,40)$, age (41), BMI $(35,37,42,43)$, body composition (44), blood lipids (45) and gut microbial composition $(46,47)$. In these studies, transcriptome analyses were performed with the aim of increasing our understanding of the effect of these characteristics and the mechanisms underlying the differences in the response to diet. In chapter 2, we review these studies and discuss the effects of these characteristics on the whole genome gene expression response to diet.

\section{Outline of this thesis}

In this thesis, we aimed to increase our understanding of the molecular mechanisms underlying the effect of dietary fatty acids. To do this, changes in whole genome gene expression profiles upon both acute as well as chronic dietary interventions with fatty acids were examined. We gave specific emphasis to differences in gene expression responses between groups of subjects that had different genotypic or phenotypic characteristics.

Chapter 2 gives an overview of all currently published studies that examined the role of genotypic and phenotypic characteristics on the whole genome gene expression response to dietary interventions. A genotypic characteristic that has not been studied before in this context is examined in chapter 3. Here, we used the PBMC transcriptome to study differences between carriers and non-carriers of APOE4 in PBMCs and we examined the differences between carriers and non-carriers in the response to a 6-month fish-oil supplementation intervention. In chapter 4 , we elucidated potential mechanisms that may explain the beneficial effects of medium-chain saturated fatty acids on body fat accumulation. We used transcriptomics in the adipose tissue to examine differences between a 12-week high medium-chain SFA and a low medium-chain SFA intervention. Chapter 5 and chapter 6 were focused on high-fat challenges in combination with whole genome gene expression measurements. In chapter 5 , we examined the potential use of high-fat challenges combined with transcriptomics to measure subtle changes in metabolic flexibility. Specifically, we studied if healthy subjects showed a shift in challenge response after a 4 week high-fat high-calorie diet towards a more metabolic syndrome-like challenge response. In chapter 6 , we studied whether a general gene expression response to a high-fat challenge can be identified. This was done by performing a meta-analysis of several previously published high-fat challenge studies that performed whole 
genome gene expression measurements in white blood cells. In chapter 7 the results described in chapter 2-6 are discussed.

\section{References}

1. World Health Organization. Global status report on noncommunicable diseases 2014. Geneva: World Health Organization; 2014. 280 p. p.

2. Joint WHO/FAO Expert Consultation on Diet Nutrition and the Prevention of Chronic Diseases (2002 : Geneva Switzerland)., World Health Organization. Dept. of Nutrition for Health and Development. Diet, nutrition and the prevention of chronic diseases : report of a joint WHO/FAO expert consultation, Geneva, 28 January-1 February 2002. Geneva: World Health Organization; 2003. 149 p. p.

3. World Health Organization. Global action plan for the prevention and control of noncommunicable diseases 2013-2020. (in IRIS). Geneva: World Health Organization; 2013. iii, 103 p. p.

4. Willett WC. Dietary fats and coronary heart disease. Journal of internal medicine. 2012;272(1):13-24.

5. Ley SH, Hamdy O, Mohan V, Hu FB. Prevention and management of type 2 diabetes: dietary components and nutritional strategies. Lancet. 2014;383(9933):1999-2007.

6. USDA National Nutrient Database for Standard Reference, Release 28 [Internet].

7. Mumme K, Stonehouse W. Effects of medium-chain triglycerides on weight loss and body composition: a metaanalysis of randomized controlled trials. J Acad Nutr Diet. 2015;115(2):249-63.

8. Bueno NB, de Melo IV, Florencio TT, Sawaya AL. Dietary medium-chain triacylglycerols versus long-chain triacylglycerols for body composition in adults: systematic review and meta-analysis of randomized controlled trials. J Am Coll Nutr. 2015;34(2):175-83.

9. Vinolo MA, Rodrigues HG, Nachbar RT, Curi R. Regulation of inflammation by short chain fatty acids. Nutrients. 2011;3(10):858-76.

10. Arora T, Backhed F. The gut microbiota and metabolic disease: current understanding and future perspectives. Journal of internal medicine. 2016;280(4):339-49.

11. Woting A, Blaut M. The Intestinal Microbiota in Metabolic Disease. Nutrients. 2016;8(4):202.

12. Tan J, McKenzie C, Potamitis M, Thorburn AN, Mackay CR, Macia L. The role of short-chain fatty acids in health and disease. Advances in immunology. 2014;121:91-119.

13. Hooper L, Martin N, Abdelhamid A, Davey Smith G. Reduction in saturated fat intake for cardiovascular disease. The Cochrane database of systematic reviews. 2015(6):CD011737.

14. de Souza RJ, Mente A, Maroleanu A, Cozma AI, Ha V, Kishibe T, et al. Intake of saturated and trans unsaturated fatty acids and risk of all cause mortality, cardiovascular disease, and type 2 diabetes: systematic review and meta-analysis of observational studies. Bmj. 2015;351:h3978.

15. Jakobsen MU, O’Reilly EJ, Heitmann BL, Pereira MA, Balter K, Fraser GE, et al. Major types of dietary fat and risk of coronary heart disease: a pooled analysis of 11 cohort studies. The American journal of clinical nutrition. 2009;89(5):1425-32.

16. Schwingshackl L, Hoffmann G. Monounsaturated fatty acids and risk of cardiovascular disease: synopsis of the evidence available from systematic reviews and meta-analyses. Nutrients. 2012;4(12):1989-2007.

17. Swanson D, Block R, Mousa SA. Omega-3 fatty acids EPA and DHA: health benefits throughout life. Advances in nutrition. 2012;3(1):1-7.

18. Jong MC, Hofker MH, Havekes LM. Role of ApoCs in lipoprotein metabolism: functional differences between ApoC1, ApoC2, and ApoC3. Arteriosclerosis, thrombosis, and vascular biology. 1999;19(3):472-84. 
19. Mahley RW, Innerarity TL. Lipoprotein receptors and cholesterol homeostasis. Biochimica et biophysica acta. 1983;737(2):197-222.

20. Weisgraber KH. Apolipoprotein E: structure-function relationships. Advances in protein chemistry. 1994;45:249-302.

21. Eisenberg DT, Kuzawa CW, Hayes MG. Worldwide allele frequencies of the human apolipoprotein E gene: climate, local adaptations, and evolutionary history. American journal of physical anthropology. 2010;143(1):100-11.

22. Corder EH, Saunders AM, Strittmatter WJ, Schmechel DE, Gaskell PC, Small GW, et al. Gene dose of apolipoprotein E type 4 allele and the risk of Alzheimer's disease in late onset families. Science. 1993;261(5123):921-3.

23. Saunders AM, Strittmatter WJ, Schmechel D, George-Hyslop PH, Pericak-Vance MA, Joo SH, et al. Association of apolipoprotein E allele epsilon 4 with late-onset familial and sporadic Alzheimer's disease. Neurology. 1993;43(8):1467-72.

24. Song Y, Stampfer MJ, Liu S. Meta-analysis: apolipoprotein E genotypes and risk for coronary heart disease. Ann Intern Med. 2004;141(2):137-47.

25. Bennet AM, Di Angelantonio E, Ye Z, Wensley F, Dahlin A, Ahlbom A, et al. Association of apolipoprotein E genotypes with lipid levels and coronary risk. JAMA. 2007;298(11):1300-11.

26. Khan TA, Shah T, Prieto D, Zhang W, Price J, Fowkes GR, et al. Apolipoprotein E genotype, cardiovascular biomarkers and risk of stroke: systematic review and meta-analysis of 14,015 stroke cases and pooled analysis of primary biomarker data from up to 60,883 individuals. Int J Epidemiol. 2013;42(2):475-92.

27. Georgiadi A, Kersten S. Mechanisms of gene regulation by fatty acids. Advances in nutrition. 2012;3(2):127-34.

28. Bouwens M, van de Rest O, Dellschaft N, Bromhaar MG, de Groot LC, Geleijnse JM, et al. Fish-oil supplementation induces antiinflammatory gene expression profiles in human blood mononuclear cells. The American journal of clinical nutrition. 2009;90(2):415-24.

29. Myhrstad MC, Ulven SM, Gunther CC, Ottestad I, Holden M, Ryeng E, et al. Fish oil supplementation induces expression of genes related to cell cycle, endoplasmic reticulum stress and apoptosis in peripheral blood mononuclear cells: a transcriptomic approach. J Intern Med. 2014;276(5):498-511.

30. van Dijk SJ, Feskens EJ, Bos MB, de Groot LC, de Vries JH, Muller M, et al. Consumption of a high monounsaturated fat diet reduces oxidative phosphorylation gene expression in peripheral blood mononuclear cells of abdominally overweight men and women. The Journal of nutrition. 2012;142(7):1219-25.

31. Wisse BE. The inflammatory syndrome: the role of adipose tissue cytokines in metabolic disorders linked to obesity. Journal of the American Society of Nephrology : JASN. 2004;15(11):2792-800.

32. Tilg H, Moschen AR. Adipocytokines: mediators linking adipose tissue, inflammation and immunity. Nature reviews Immunology. 2006;6(10):772-83.

33. Weisberg SP, McCann D, Desai M, Rosenbaum M, Leibel RL, Ferrante AW, Jr. Obesity is associated with macrophage accumulation in adipose tissue. The Journal of clinical investigation. 2003;112(12):1796-808.

34. van Dijk SJ, Feskens EJ, Bos MB, Hoelen DW, Heijligenberg R, Bromhaar MG, et al. A saturated fatty acid-rich diet induces an obesity-linked proinflammatory gene expression profile in adipose tissue of subjects at risk of metabolic syndrome. The American journal of clinical nutrition. 2009;90(6):1656-64.

35. Esser D, van Dijk SJ, Oosterink E, Lopez S, Muller M, Afman LA. High fat challenges with different fatty acids affect distinct atherogenic gene expression pathways in immune cells from lean and obese subjects. Mol Nutr Food Res. 2015;59(8):1563-72.

36. Bouwens M, Grootte Bromhaar M, Jansen J, Muller M, Afman LA. Postprandial dietary lipid-specific effects on human peripheral blood mononuclear cell gene expression profiles. The American journal of clinical nutrition. 2010;91(1):208-17. 
37. Matone A, O'Grada CM, Dillon ET, Morris C, Ryan MF, Walsh M, et al. Body mass index mediates inflammatory response to acute dietary challenges. Mol Nutr Food Res. 2015;59(11):2279-92.

38. Stroeve JH, van Wietmarschen H, Kremer BH, van Ommen B, Wopereis S. Phenotypic flexibility as a measure of health: the optimal nutritional stress response test. Genes \& nutrition. 2015;10(3):459.

39. Camargo A, Ruano J, Fernandez JM, Parnell LD, Jimenez A, Santos-Gonzalez M, et al. Gene expression changes in mononuclear cells in patients with metabolic syndrome after acute intake of phenol-rich virgin olive oil. BMC genomics. 2010;11:253.

40. Rudkowska I, Paradis AM, Thifault E, Julien P, Tchernof A, Couture P, et al. Transcriptomic and metabolomic signatures of an n-3 polyunsaturated fatty acids supplementation in a normolipidemic/normocholesterolemic Caucasian population. The Journal of nutritional biochemistry. 2013;24(1):54-61.

41. Thalacker-Mercer AE, Fleet JC, Craig BA, Campbell WW. The skeletal muscle transcript profile reflects accommodative responses to inadequate protein intake in younger and older males. The Journal of nutritional biochemistry. 2010;21(11):1076-82.

42. van Erk MJ, Pasman WJ, Wortelboer HM, van Ommen B, Hendriks HF. Short-term fatty acid intervention elicits differential gene expression responses in adipose tissue from lean and overweight men. Genes Nutr. 2008;3(3-4):127-37.

43. Pasman WJ, van Erk MJ, Klopping WA, Pellis L, Wopereis S, Bijlsma S, et al. Nutrigenomics approach elucidates health-promoting effects of high vegetable intake in lean and obese men. Genes Nutr. 2013;8(5):507-21.

44. Radonjic M, van Erk MJ, Pasman WJ, Wortelboer HM, Hendriks HF, van Ommen B. Effect of body fat distribution on the transcription response to dietary fat interventions. Genes Nutr. 2009;4(2):143-9.

45. Schmidt S, Stahl F, Mutz KO, Scheper T, Hahn A, Schuchardt JP. Different gene expression profiles in normoand dyslipidemic men after fish oil supplementation: results from a randomized controlled trial. Lipids in health and disease. 2012;11:105.

46. Niculescu MD, Pop EA, Fischer LM, Zeisel SH. Dietary isoflavones differentially induce gene expression changes in lymphocytes from postmenopausal women who form equol as compared with those who do not. The Journal of nutritional biochemistry. 2007;18(6):380-90.

47. van der Velpen V, Geelen A, Hollman PC, Schouten EG, van 't Veer P, Afman LA. Isoflavone supplement composition and equol producer status affect gene expression in adipose tissue: a double-blind, randomized, placebo-controlled crossover trial in postmenopausal women. The American journal of clinical nutrition. 2014;100(5):1269-77. 


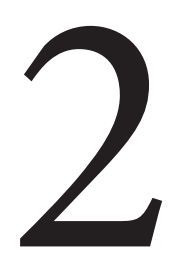

\section{The use of transcriptomics as a tool to identify differences in the response to diet}

Juri Matualatupauw and Lydia Afman

In Kussmann M and Stover PJ (Eds.), Nutrigenomics and Proteomics in Health and Disease: Towards a Systems-Level Understanding of Gene-Diet Interactions (pp.1-18).

Chichester, UK: John Wiley \& Sons. 


\section{New concepts in nutrition research}

The role of nutrition in the pathogenesis of metabolic diseases, such as type 2 diabetes and cardiovascular disease, is clearly recognized. In the past, nutritional research has aimed to provide general dietary advice aimed at improving population health. Problematic with this approach is that even though dietary changes may be of great benefit at population level, the effects on individual level are very small and hardly noticeable (1). The ultimate way to improve health is by providing personalized dietary advice. New approaches and methodologies are essential if we want to demonstrate nutritional effects on health at the individual level. The main challenges we are facing within the nutrition field are the high variability in response to nutrition between subjects, the relative small effects of nutrition and the long period it may take before effects become evident. One of the key issues in the high variability in response is that not only non-mutable factors such as age, gender and genotype are affecting the response. Changeable factors such as health status are also affecting response to nutrition. The drawback with the latter is the lack of appropriate biomarkers to characterize individual health status. The markers used to show efficacy of interventions are often late single biomarkers of disease state. These biomarkers are relevant to demonstrate efficacy of pharmacological interventions but are less applicable to show efficacy of nutritional interventions, that are mostly performed in a relatively healthy population.

\section{Comprehensive phenotyping}

A new concept in nutrition research is the measurement of a wide range of markers to characterize health, which is called 'comprehensive phenotyping' (2). The arrival of comprehensive genomics techniques in the last decade drove this development, as it enabled measurement of expression of thousands of genes, proteins and metabolites in one sample. These techniques can be applied in a range of samples including blood, urine, cells and tissue biopsies, that can quite easily be collected during dietary intervention studies in healthy volunteers. The last couple of years we have demonstrated the sensitivity of these techniques by showing nutritional effects on health where classical approaches failed $(3,4)$. Comprehensive phenotyping not only includes -omics techniques but also requires the measurement of classical markers and intermediary endpoint measures that have been shown to be associated with disease. Better characterization of health status by using a comprehensive phenotyping approach not only helps to demonstrate efficacy of a nutritional intervention, it also supports the identification of people at risk for disease development that still can profit from dietary 
advice.

Comprehensive phenotyping is still in an early phase. Very few studies have been published so far that integrated omics techniques with functional and classical markers in the field of nutrition. Very recently, a study has been published in which a huge amount of data has been integrated to characterize individual responses to nutrition (5). The ultimate goal was to develop a machine-learning algorithm that predicts personal postprandial glycemic responses to real-life meals. Week-long glucose levels and responses to 46,898 meals were continuously measured in a cohort of 800 people. This study adopted a comprehensive phenotyping approach by integrating the glucose responses with blood parameters, dietary habits, anthropometrics, physical activity, and gut microbiota. The predictions of postprandial glycemic responses were validated in an independent 100-person cohort. Furthermore, a blinded randomized controlled dietary intervention based on this algorithm resulted in significantly lower postprandial glucose responses and consistent alterations to gut microbiota composition. This study shows that with the use of comprehensive phenotyping and adequate data integration, personalized nutrition is potentially within our reach.

\section{Phenotypic flexibility}

Another new development within the nutrition field is the measurement of an individual's capacity to adapt to dietary challenges, which is called 'phenotypic flexibility' $(2,6,7)$. A dietary challenge, such as a high-fat challenge or an oral glucose tolerance test (OGTT), triggers the adaptation capacity of organs, cells and tissues, and challenges metabolic and inflammatory homeostasis. For example, oral high-fat challenges have been used to study postprandial lipid metabolism showing a high variation in individual responses. Individuals with a more pronounced postprandial response were at an increased risk of developing CVD. Similarly, an OGTT is used to evaluate insulin resistance. At fasting, insulin insensitivity maybe not detectable, but after an OGGT insulin insensitivity becomes apparent. Phenotypic flexibility can be an important indicator of individual health status, as it might reflect the (dys-)functioning of metabolic organs, such as liver and adipose tissue. It might therefore be able to better characterize health status or reveal effects of nutrition on health that otherwise would have remained undetected.

The combination of both approaches, i.e. comprehensive phenotyping and phenotypic flexibility, will result in a dynamic biomarker profile as outcome measure. This profile is expected to provide more information on health status and thus efficacy of dietary 
interventions than the static single biomarkers that have been used so far.

Studies using a comprehensive phenotyping approach to characterize individual responses to diet are rare. Most studies that examined individual responses to diet using comprehensive omics techniques performed these analyzes retrospectively and only few studies stratified groups beforehand. The same scarcity accounts for studies that used challenge tests in combination with omics techniques to characterize individual responses based on phenotype.

In this chapter we will summarize the studies that either used non-mutable factors such as age, gender and genotype or mutable factors such as health status to characterize individual response to diet, long or medium-term or after a nutritional challenge. Specific focus will be on papers that used the comprehensive -omics technique transcriptomics as outcome measure.

\section{Factors that influence the transcriptome response to diet}

Transcriptomics is one of the first of the omics-technologies that was used in nutritionrelated research in humans. Much of the research has focused on examining changes in gene expression patterns using microarrays, either upon acute challenges or upon longer-term dietary interventions. One of the types of cells that is frequently used to asses transcriptome profiles are blood cells, which are easy and non-invasive to harvest in humans. A subpopulation of blood immune cells regularly studied are peripheral blood mononuclear cells (PBMCs). Subcutaneous adipose tissue is also often studied in human nutrigenomics studies, because it is relatively non-invasive to take biopsies from this tissue and adipose tissue is known to play a key role in the pathogenesis of metabolic diseases. Lastly, skeletal muscle has also been examined in some studies.

Several studies that investigated the change in whole-genome gene expression upon a nutritional intervention observed large inter-individual differences in response to a dietary intervention (8-11). The reasons for these large inter-individual differences are not yet fully understood, but can range from genetic, phenotypic or environmental differences between individuals. Of particular interest in the context of personalized nutrition are the studies that identified factors that have an interaction effect on the response to diet. This chapter will focus on studies that examined this interaction effect using transcriptomics as outcome measure. Factors that will be discussed are gender, age, genotype, anthropometric measurements, plasma biochemical markers and gut microbiota. Furthermore, we will discuss some studies 
that used other outcome measures to identify responders and non-responders to diet and subsequently used transcriptomics to mechanistically examine the differences between these two groups.

\section{Gender}

Gender is one of the most obvious phenotypes of which a difference in response to diet can be expected. Still, the number of studies that investigated the difference in gene expression response to diet between men and women is limited. One study examined the postprandial changes in PBMC gene expression after a breakfast based on olive oil with a high or low amount of phenol compounds (12). Microarray analysis demonstrated a significant change in expression of 98 genes between the high-phenol and the low-phenol breakfast. However, when performing additional separate analyses for men and women, they found a higher number of differentially expressed genes; 250 and 143, respectively. Only 32 genes were differentially expressed in both men and women, indicating that the effect of the phenols on PBMC gene expression might be affected by gender.

A study by Rudkowska et al. (2013) (13) examined the effects of 6 weeks of n-3 polyunsaturated fatty acids (PUFAs) supplementation on PBMC gene expression in 29 overweight and obese men and women. Microarrays showed that 170 transcripts were differentially expressed upon n-3 PUFA when examining gene expression changes in the total study population. However, when separate analyses for men $(n=12)$ and women $(n=17)$ were performed, 610 transcripts were differentially expressed in men and 205 in women. Only nine transcripts overlapped between men and women, indicating that the gene expression response in PBMCs to n-3 PUFAs may be different between men and women. Pathways differentially expressed between men and women were related to oxidative stress, PPAR-alpha signaling and NF-kB signaling. Expression of genes in the oxidative stress and PPAR-alpha signaling pathways were downregulated in men and upregulated in women, whereas genes in the NF-kB signaling pathway were downregulated in men only.

Taken together, these two studies indicate that the gene expression response to certain nutrients is influenced by gender. Even though this seems to be a very plausible assertion, many studies do not differentiate between men and women and the two above-mentioned studies only did this in a secondary analysis.

\section{Age}


Another obvious factor that may cause a difference in response to diet is age. Many studies already take age into account by only selecting subjects in certain age groups. We identified only one study that actually examined the effect of age on the whole genome gene expression response to diet. In this study, Thalacker-Mercer et al (2010) (14) performed a cross-over trial in which 12 younger (21-43 years) and 10 older (63-79 years) healthy men were given a controlled diet containing a high, medium or low amount of protein for three 18-day periods. Microarrays were performed on skeletal muscle biopsies that were taken on day 12 of each intervention period. A significant interaction between diet and age was observed for 853 genes. With increasing protein in the diet, expression of genes related to protein metabolism was found to increase in younger subjects and decrease in older subjects. Moreover, older men had an increased expression of genes related to protein catabolism on the low-protein diet. Previously, older subjects showed a reduced anabolic response in skeletal muscle to increased protein intake compared to younger subjects (15). It is known that protein needs are indeed different between young and old. Using transcriptomics, Thalacker-Mercer et al tried to identify processes that take place in the muscle that may be responsible for this. Besides the effects of protein in muscle, it is conceivable that age may also affect the response to other nutrients and on other tissues.

\section{Genotype}

One of the most studied feature of personalized nutrition is gene-diet interactions, where researchers examine the effects of gene variants on the response to diet. This area of research is referred to as nutrigenetics in the scientific literature. It is clear that part of the individual differences in the response to diet are caused by genetic differences. Research has focused on examining the effects of gene variants of several genes, some of the most-studied genes being: APOA5, APOE, GST, MTHFR and PLIN (16). These studies, however, focus mostly on the effects of these gene variants on blood biomarkers or disease outcomes. Omics technologies may be very useful for better characterization of the effects of some of these gene variants and to understand underlying mechanisms (17). However, to our knowledge, no studies have investigated gene-diet interactions using a transcriptomics approach.

\section{Anthropometric measurements}

Besides non-changeable phenotypes such as gender, age and genotype, other factors may also affect the response to diet. One of these factors is BMI. We performed a study in which we examined the effect of BMI on the postprandial transcription response to a high-fat shake 
(18). In a cross-over design, 17 lean and 15 obese subjects consumed shakes containing 95g of fat, enriched in either saturated fatty acids (SFAs) or monounsaturated fatty acids (MUFAs). Microarrays were used to examine changes in whole-genome gene expression in PBMCs before and after intake of the two shakes. We observed marked differences in the response to these high-fat challenges when comparing obese to lean subjects, with 607 and 2516 genes being differentially expressed after the SFA-shake and the MUFA-shake respectively. In response to the SFA-challenge, genes related to platelet activation were upregulated in obese and downregulated in lean subjects. In response to the MUFA-challenge, genes related to post-translational protein modification were upregulated in obese and downregulated in lean subjects. Genes related to G-protein coupled receptors were downregulated in obese and upregulated in lean subjects.

Another study also examined the effect of BMI on postprandial gene expression response to a high-fat challenge and a high-glucose challenge (19). In this cross-over study, a subgroup of 23 subjects underwent both the high-fat as well as the high-glucose challenge. PBMC gene expression profiles were determined before and after both challenges. The authors found that some genes showed a consistent response regardless of BMI. However, quite a large amount of genes responded in a BMI-dependent manner: 760 genes for the high-fat and 269 for the high-glucose challenge. These genes were related to T-cell receptor-mediated inflammatory signaling and cell adhesion pathways, with some of these genes being downregulated and some upregulated with increasing BMI. Moreover, the effect of BMI on the gene expression profiles was largest on the high-fat compared to the high-glucose challenge.

Besides these acute challenge studies, the effects of BMI on mid- to long-term dietary interventions were investigated as well. Pasman et al. (2013) (20) studied the effects of BMI on adipose tissue gene expression profiles upon 4 weeks of high versus low vegetable consumption. Ten lean and ten obese subjects consumed 200 or $50 \mathrm{~g}$ of vegetables daily in a cross-over study design. When comparing the high to the low vegetable intake, 532 genes were found to be differentially expressed in lean subjects and 323 genes in obese subjects. In lean subjects, enriched pathways were related to inflammation, with an increase in gene expression of IL8 and NFKB2 and a decrease in gene expression of complement component 3 and NFKB inhibitor. In the group of obese subjects, inter-individual variation in response was found to be high and consequently no pathways were found to be enriched.

In one study, a short-term intervention was performed to examine the effect of BMI on the gene expression response in adipose tissue to a 9-day nutritional intervention (21). In 
a crossover study design, subjects consumed 40g/day of either an intervention spread, containing increased amounts of medium-chain triglycerides, PUFAs and conjugated linoleic acid, or a control spread. The intervention decreased expression of genes related to energy metabolism in lean subjects only. Obese subjects showed a downregulation of inflammatory genes and an upregulation of lipid metabolism-related genes. Interestingly, inter-individual variation in the gene expression response in the obese subjects was found to be quite high. The authors performed an additional analysis, in which they found that expression of genes related to mitochondrion, cell adhesion, extracellular matrix, immune response and inflammatory response correlated better to waist-to-hip ratio and fat percentage than BMI.

In addition to BMI, the amount of fat tissue or body fat distribution may be important in determining the response to diet. In a small cross-over study, Radonjic et al. (2009) (22) studied the effect of body fat distribution on the whole genome gene expression response to two dietary fat interventions. Microarrays were performed on adipose tissue samples before and after interventions. The authors compared subjects with upper body obesity (waist-tohip ratio $>1$ ) to those with lower body obesity (waist-to-hip ratio $<1$ ). The intervention diets contained either predominantly long-chain PUFAs or medium-chain fatty acids. When comparing the effects of the two interventions on gene expression, they found more genes to be differentially expressed in upper body obese subjects; 239 genes, compared to lower body obese subjects; 73 genes. A subsequent analysis on pathway level showed that with increasing waist-to-hip ratio, expression of immune response and apoptosis-related genes increased and metabolism-related genes decreased, when comparing the medium chain fatty acid vs the PUFA-enriched diet. This study shows that there may be differences in the gene expression response to dietary fatty acids between upper body obese and lower body obese subjects. However, the number of subjects in this study was low with 5 upper body obese subjects and 6 lower body obese subjects, so care should be taken with interpretation of the results.

In summary, BMI has been shown to affect acute postprandial gene expression response to different types of acute challenges in PBMCs. These effects were mainly observed in pathways related to inflammation and cell adhesion. Moreover, the effect of BMI on gene expression changes was found to be stronger for a fatty acid challenge compared to a glucose challenge. For short to medium-term dietary interventions, there is some evidence that BMI as well as body fat distribution may affect the response of the subcutaneous adipose tissue to diets containing different types and amounts of fatty acids. Lastly, waist-to-hip ratio and fat percentage may explain a larger percentage of the inter-individual differences in response to 
nutritional interventions than BMI.

\section{Plasma biochemical markers}

High levels of triglycerides, LDL-cholesterol and total cholesterol as well as low HDLcholesterol levels in the blood are associated with an increased risk of CVD. Understanding how persons with different levels of these biomarkers respond to dietary interventions could be very useful in preventing disease. One study used a transcriptomic approach to examine the effects of a 12-week fish-oil and corn-oil supplementation in normo- and dyslipidemic men (total cholesterol > 200mg/dl, LDL cholesterol > $130 \mathrm{mg} / \mathrm{dl}$, triglycerides $>150 \mathrm{mg} /$ dl) (23). Microarrays were used to study whole blood cell gene expression. Substantially more genes were differentially expressed by 12 -week consumption of both types of oils in dyslipidemic men compared to normolipidemic men. Fish-oil supplementation regulated genes related to immune system, inflammation, lipid metabolism and cardiovascular disease in the dyslipidemic subjects. Expression of several genes related to fatty acid metabolism were downregulated, emphasizing the potential beneficial value of n-3 PUFAs in dyslipidemic persons.

\section{Gut microbiota}

The link between the gut microbiota and the development of obesity, cardiovascular disease and type 2 diabetes has received much attention in recent years (24). It has become clear that the microbes in our gut can affect the way we respond to nutrients. One of the nutrients that has been studied in relation to the gut microbiome are isoflavones. Isoflavones are compounds that are naturally present in soy and are structurally very similar to the $17 \beta$-estradiol hormone. The effects of isoflavones are, in part, mediated by their binding to estrogen receptors (25). Therefore, isoflavone supplemention might be of interest during and after menopause. In women, isoflavones are thought to have positive health effects on menopausal complaints, such as hot flashes (26). One of the major soy isoflavones, daidzein, is converted to equol by intestinal bacteria. Of all humans, 30-60\% carry these bacteria and are equol producers. Equol has a higher estrogenic and antioxidant activity compared to daidzein and other isoflavones. Due to these properties, it is hypothesized that isoflavones supplementation is especially beneficial in equol producers (27).

Niculescu et al. (2006) (28) performed a study, which was designed to examine the effect of equol producer status on isoflavone supplementation-induced changes in gene expression in blood lymphocytes. Postmenopausal equol-producing and non-producing women showed a 
similar number of differentially expressed genes after 84 days of soy isoflavone supplementation compared to placebo, 319 versus 322 respectively. However, equol-producing women had an increased expression of estrogen-responsive genes compared to non-producers, illustrating the importance of equol-producer status in modulating estrogen-related actions of isoflavones. We also studied the effect of equol-producer status on whole-genome gene expression in the adipose tissue of post-menopausal women upon 8 weeks consumption of two different commercially available isoflavone supplements that were either low or high in genistein (29). For the low-genistein supplements, 883 and 1169 genes were differentially regulated in non equol-producers and equol producers respectively, whereas for the high-genistein supplements, 547 and 631 genes were differentially regulated for non equol-producers and equol producers respectively. Independent of supplement type, expression of energy metabolism-related genes was downregulated in equol producers and upregulated in nonproducers after supplementation. Furthermore, equol producers showed an anti-inflammatory gene expression response to the two types of isoflavone supplements whereas this response was not observed in non-producers.

In summary, the effects of the gut microbiome on whole-genome gene expression have been studied only in the specific case of equol producing bacteria. The transcriptomics studies point towards more pronounced effects of isoflavones in equol producing postmenopausal women. Much remains to be studied with regard to gut microbiome-diet interactions.

\section{Using transcriptomics to explain the mechanism behind differences in response to diet}

Transcriptomics has also been used to better understand the differences between responders and non-responders to interventions. Shike et al. (2014) (30) studied the effects of soy supplementation on gene expression in patients with invasive breast cancer. Patients were randomly assigned to soy supplementation $(n=70)$ or placebo $(n=70)$ for the period from diagnosis until surgery, which ranged from 7 to 30 days. Genome-wide gene expression was measured post-treatment in surgically resected tumor samples of a larger group $(n=35)$ of patients using microarrays. In a secondary analysis, the authors compared the gene expression response between high and low responders to the intervention based on serum genistein levels. They compared tumor gene expression of a high-genistein level subset of patients $(n=12)$ to a subset of patients with low genistein levels $(n=23) .126$ genes were differentially 
expressed between these two groups and pathway analysis revealed an increased expression of pathways related to cell growth and proliferation in the tumors of the high-genistein patients. Moreover, expression of FGFR2, a known oncogene and marker of poor prognosis in breast cancer (31), was increased in the high-genistein compared to the low genistein group. Taken together, this study provides indications that soy supplementation may not be beneficial in all breast cancer patients and identifies a subgroup of patients that show a high-genistein response in which soy supplementation may actually be harmful.

Rudkowska et al. (2012) (32) compared PBMC transcriptomic profiles of responders and non-responders to a 6 week n-3 PUFA supplementation. Six subjects in which plasma triglycerides were lowered by n-3 PUFAs (responders) were matched to 6 subjects in which they were not (non-responders). Several genes related to lipid metabolism were differentially expressed between responders and non-responders. These results indicate that there may be some differences in the way lipids are handled between the two groups.

A study by Mutch et al. (2011) (33) investigated differences in gene expression profiles between subjects that maintained weight loss versus those that regained weight after a period of caloric restriction. They compared changes in whole genome gene expression profiles in subcutaneous adipose tissue upon caloric restriction in the two groups and found 1291 and 1298 genes differentially expressed by caloric restriction within weight maintainers and weight regainers respectively. Weight maintainers showed decreases in expression of genes related to extracellular matrix, whereas the weight regainers showed increased expression in these genes. Moreover, weight maintainers increased their expression of genes related to apoptosis and p53, whereas the weight regainers showed no change in expression of these genes. In conclusion, this study reveals differences in gene expression profiles between weight maintainers and regainers and provides some leads in understanding the causes of successful weight maintenance.

In summary, these studies show that gene expression profiles can be used to better understand why some persons do respond favorably to a dietary intervention and others do not.

\section{Conclusion}

In this chapter, we have discussed studies that used transcriptomics for studying differences in responses to diet. These studies point towards clear differences in the gene expression response to diet based on phenotypic measurements. In summary, transcriptomics-based studies 
have shown that several factors can influence the gene expression response to diet, which are gender, age, genotype, anthropometric measurements, plasma biochemical markers and gut microbiota. Besides using the transcriptome itself as an outcome measure, some studies have used transcriptomics to examine mechanistic differences between responders and nonresponders to dietary interventions on other outcome measures. This approach provides insight into which genes and pathways are involved and provides mechanistic understanding on the response to nutrients and diets.

BMI is the most extensively studied factor and substantial evidence shows that BMI affects the transcriptome responses to diet, both in acute challenge studies as well as in short- to medium-term dietary interventions. However, for the other factors, too few studies were performed to draw definitive conclusions. Furthermore, most of these studies were designed with a different primary research question and only examined these interaction-factors in secondary analyses. As a consequence, the numbers of subjects is generally very low, leading to underpowered studies.

\section{Future perspectives}

What is missing so far are studies that are specifically designed to study factors that may explain differences in response to diet. Only for BMI such studies could be identified. In the future, studies with stratification by these factors on forehand could help in unraveling the role of these factors in diet-induced personal responses on the transcriptome.

Designing studies that specifically address one of these factors may be useful, though a major drawback with this approach is that numerous factors influence the response to diet and the most important interaction-factors may not be known in advance. A more complete approach would be to perform large studies that use a combination of the two new concepts, i.e. phenotypic flexibility and comprehensive phenotyping. This will enable advanced characterization of personal responses to diets, especially if applied before and after a dietary intervention. These type of studies allow identification of a combination of characteristics responsible for a personal dietary response. Transcriptomics may play an additional role by mechanistically explaining the individual differences in response to diet, which was also shown in the studies discussed above.

However, before we are able to characterize personal response to diet we first need to know how robust the personal response to diet is. Studies measuring the response to the same 
repeated dietary exposure are missing but are essential for personalized dietary advice. Many variables known to affect the transcriptome response, may influence this personal response to diet such as sleep deprivation, stress, or physical activity. Standardization and comprehensive measurements of relevant factors are key in this respect. Once the robustness of a personal response to diet is defined, the next question is whether a health status profile of or signature can be identified that can predict this response to diet, using approaches such as the machinelearning algorithm method performed by Zeevi et al. (2015) (5). The last phase is to unravel what the best diet is to improve the personal health status as defined by the health status profile or signature, i.e. personalized dietary advice. For the latter (issue), extensive evaluation of the profile is of high importance; what kind of biomarkers are in the profile, what do they reflect, where do they derive from and which metabolic routes or pathways in the body might be affected. In addition, what is known about the effects of nutrients and diet on these pathways and routes in these organs or cells, and how can we influence these routes or pathways by nutrition? It is known that nutrients can very subtly regulate gene expression of metabolic routes and pathways via binding to and activation of transcription factors (34). Integration and interpretation of the data enables the discovery and quantification of processes important for health that can be targeted by nutrition.

In summary, although a lot of research needs to be done before we are able to give personalized dietary advice based on the health status of a person, the techniques are available. They have been applied and have also shown to be sensitive enough to identify personal responses to diet. Application of the phenotypic flexibility concept in combination with comprehensive phenotyping both before and after dietary interventions is promising, as it might deliver more information on individual responses to diet and health status markers. The biggest challenge for the future is the integration of all data available and the biological interpretation of the data with the ultimate goal to provide personalized dietary advice.

\section{References}

1. Rose G. Sick individuals and sick populations. International journal of epidemiology. 1985;14(1):32-8.

2. van Ommen B, Keijer J, Heil SG, Kaput J. Challenging homeostasis to define biomarkers for nutrition related health. Molecular nutrition \& food research. 2009;53(7):795-804.

3. Bouwens M, van de Rest O, Dellschaft N, Bromhaar MG, de Groot LC, Geleijnse JM, et al. Fish-oil supplementation induces antiinflammatory gene expression profiles in human blood mononuclear cells. The American journal of clinical nutrition. 2009;90(2):415-24.

4. van Dijk SJ, Feskens EJ, Bos MB, Hoelen DW, Heijligenberg R, Bromhaar MG, et al. A saturated fatty acid-rich 
diet induces an obesity-linked proinflammatory gene expression profile in adipose tissue of subjects at risk of metabolic syndrome. The American journal of clinical nutrition. 2009;90(6):1656-64.

5. Zeevi D, Korem T, Zmora N, Israeli D, Rothschild D, Weinberger A, et al. Personalized Nutrition by Prediction of Glycemic Responses. Cell. 2015;163(5):1079-94.

6. Stroeve JH, van Wietmarschen H, Kremer BH, van Ommen B, Wopereis S. Phenotypic flexibility as a measure of health: the optimal nutritional stress response test. Genes \& nutrition. 2015;10(3):459.

7. van Ommen B, van der Greef J, Ordovas JM, Daniel H. Phenotypic flexibility as key factor in the human nutrition and health relationship. Genes \& nutrition. 2014;9(5):423.

8. Whitney AR, Diehn M, Popper SJ, Alizadeh AA, Boldrick JC, Relman DA, et al. Individuality and variation in gene expression patterns in human blood. Proceedings of the National Academy of Sciences of the United States of America. 2003;100(4):1896-901.

9. Eady JJ, Wortley GM, Wormstone YM, Hughes JC, Astley SB, Foxall RJ, et al. Variation in gene expression profiles of peripheral blood mononuclear cells from healthy volunteers. Physiological genomics. 2005;22(3):402-11.

10. Radich JP, Mao M, Stepaniants S, Biery M, Castle J, Ward T, et al. Individual-specific variation of gene expression in peripheral blood leukocytes. Genomics. 2004;83(6):980-8.

11. van Erk MJ, Blom WA, van Ommen B, Hendriks HF. High-protein and high-carbohydrate breakfasts differentially change the transcriptome of human blood cells. The American journal of clinical nutrition. 2006;84(5):1233-41.

12. Camargo A, Ruano J, Fernandez JM, Parnell LD, Jimenez A, Santos-Gonzalez M, et al. Gene expression changes in mononuclear cells in patients with metabolic syndrome after acute intake of phenol-rich virgin olive oil. BMC genomics. 2010;11:253.

13. Rudkowska I, Paradis AM, Thifault E, Julien P, Tchernof A, Couture P, et al. Transcriptomic and metabolomic signatures of an $n-3$ polyunsaturated fatty acids supplementation in a normolipidemic/normocholesterolemic Caucasian population. The Journal of nutritional biochemistry. 2013;24(1):54-61.

14. Thalacker-Mercer AE, Fleet JC, Craig BA, Campbell WW. The skeletal muscle transcript profile reflects accommodative responses to inadequate protein intake in younger and older males. The Journal of nutritional biochemistry. 2010;21(11):1076-82.

15. Katsanos CS, Kobayashi H, Sheffield-Moore M, Aarsland A, Wolfe RR. Aging is associated with diminished accretion of muscle proteins after the ingestion of a small bolus of essential amino acids. The American journal of clinical nutrition. 2005;82(5):1065-73.

16. Corella D, Ordovas JM. Aging and cardiovascular diseases: the role of gene-diet interactions. Ageing research reviews. 2014;18:53-73.

17. Morine MJ, Monteiro JP, Wise C, Teitel C, Pence L, Williams A, et al. Genetic associations with micronutrient levels identified in immune and gastrointestinal networks. Genes \& nutrition. 2014;9(4):408.

18. Esser D, van Dijk SJ, Oosterink E, Lopez S, Muller M, Afman LA. High fat challenges with different fatty acids affect distinct atherogenic gene expression pathways in immune cells from lean and obese subjects. Mol Nutr Food Res. 2015;59(8):1563-72.

19. Matone A, O'Grada CM, Dillon ET, Morris C, Ryan MF, Walsh M, et al. Body mass index mediates inflammatory response to acute dietary challenges. Molecular nutrition \& food research. 2015.

20. Pasman WJ, van Erk MJ, Klopping WA, Pellis L, Wopereis S, Bijlsma S, et al. Nutrigenomics approach elucidates health-promoting effects of high vegetable intake in lean and obese men. Genes Nutr. 2013;8(5):507-21.

21. van Erk MJ, Pasman WJ, Wortelboer HM, van Ommen B, Hendriks HF. Short-term fatty acid intervention elicits differential gene expression responses in adipose tissue from lean and overweight men. Genes Nutr. 2008;3(3-4):127-37.

22. Radonjic M, van Erk MJ, Pasman WJ, Wortelboer HM, Hendriks HF, van Ommen B. Effect of body fat 
distribution on the transcription response to dietary fat interventions. Genes Nutr. 2009;4(2):143-9.

23. Schmidt S, Stahl F, Mutz KO, Scheper T, Hahn A, Schuchardt JP. Different gene expression profiles in normoand dyslipidemic men after fish oil supplementation: results from a randomized controlled trial. Lipids in health and disease. 2012;11:105.

24. Tremaroli V, Backhed F. Functional interactions between the gut microbiota and host metabolism. Nature. 2012;489(7415):242-9.

25. Setchell KD. Soy isoflavones--benefits and risks from nature's selective estrogen receptor modulators (SERMs). Journal of the American College of Nutrition. 2001;20(5 Suppl):354S-62S; discussion 81S-83S.

26. Taku K, Melby MK, Kronenberg F, Kurzer MS, Messina M. Extracted or synthesized soybean isoflavones reduce menopausal hot flash frequency and severity: systematic review and meta-analysis of randomized controlled trials. Menopause. 2012;19(7):776-90.

27. Magee PJ. Is equol production beneficial to health? The Proceedings of the Nutrition Society. 2011;70(1):10-8.

28. Niculescu MD, Pop EA, Fischer LM, Zeisel SH. Dietary isoflavones differentially induce gene expression changes in lymphocytes from postmenopausal women who form equol as compared with those who do not. The Journal of nutritional biochemistry. 2007;18(6):380-90.

29. van der Velpen V, Geelen A, Hollman PC, Schouten EG, van 't Veer P, Afman LA. Isoflavone supplement composition and equol producer status affect gene expression in adipose tissue: a double-blind, randomized, placebo-controlled crossover trial in postmenopausal women. The American journal of clinical nutrition. 2014;100(5):1269-77.

30. Shike M, Doane AS, Russo L, Cabal R, Reis-Filho JS, Gerald W, et al. The effects of soy supplementation on gene expression in breast cancer: a randomized placebo-controlled study. Journal of the National Cancer Institute. 2014;106(9).

31. Sun S, Jiang Y, Zhang G, Song H, Zhang X, Zhang Y, et al. Increased expression of fibroblastic growth factor receptor 2 is correlated with poor prognosis in patients with breast cancer. Journal of surgical oncology. 2012;105(8):773-9.

32. Rudkowska I, Paradis AM, Thifault E, Julien P, Barbier O, Couture P, et al. Differences in metabolomic and transcriptomic profiles between responders and non-responders to an $n-3$ polyunsaturated fatty acids (PUFAs) supplementation. Genes \& nutrition. 2013;8(4):411-23.

33. Mutch DM, Pers TH, Temanni MR, Pelloux V, Marquez-Quinones A, Holst C, et al. A distinct adipose tissue gene expression response to caloric restriction predicts 6-mo weight maintenance in obese subjects. The American journal of clinical nutrition. 2011;94(6):1399-409.

34. Muller M, Kersten S. Nutrigenomics: goals and strategies. Nat Rev Genet. 2003;4(4):315-22. 


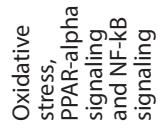

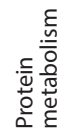

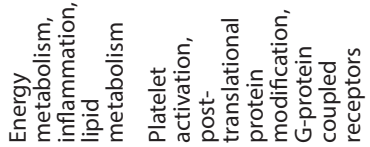

๗ั㐅 ๙

受

$\sum_{\infty} \quad \sum_{\infty}$

요

స

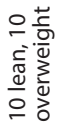

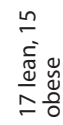

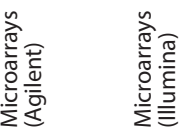

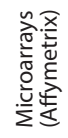

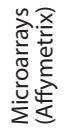

竞突

$\sum_{0}^{\ddots} \quad \sum_{0}^{\cup}$

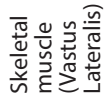

气ั๊

$\sum_{\substack{n \\ 0}}^{n}$

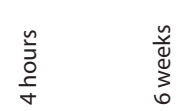

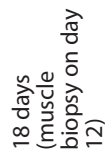

ลัก

$\stackrel{n}{5}$
$\frac{0}{\sigma}$
$\frac{5}{4}$

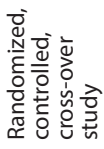

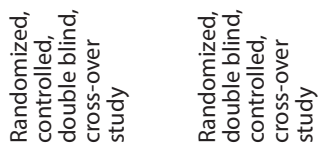

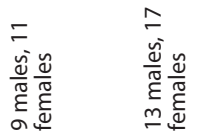

$\frac{\check{c}}{\stackrel{\frac{\pi}{\pi}}{N}}$

$\frac{\mathfrak{\varrho}}{\stackrel{\varrho}{\widetilde{E}}}$

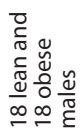

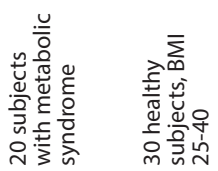

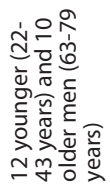

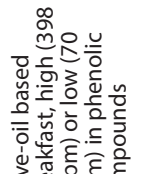

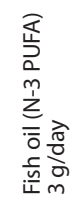

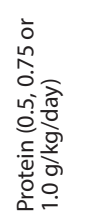

离

흥흐을응 훙

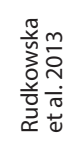

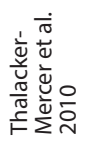

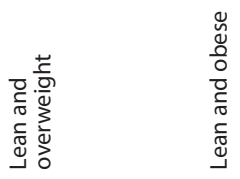

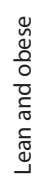

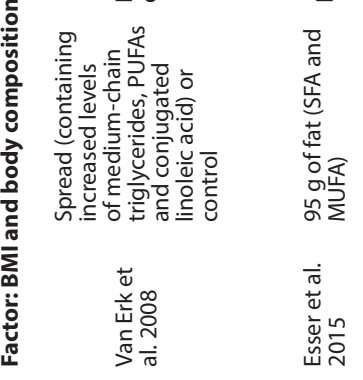




\begin{tabular}{|c|c|c|c|c|c|c|c|c|}
\hline 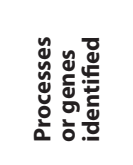 & 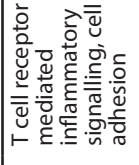 & 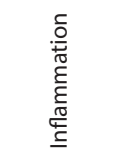 & 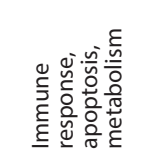 & & 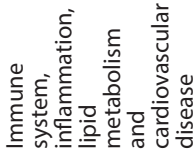 & & 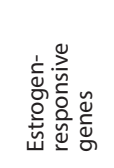 & 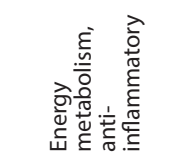 \\
\hline 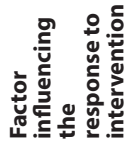 & $\sum_{\infty}$ & $\sum_{\infty}$ & 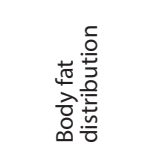 & & 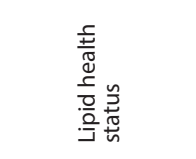 & & 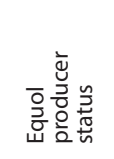 & 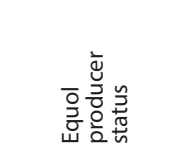 \\
\hline 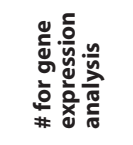 & $\ddot{\sim}$ & 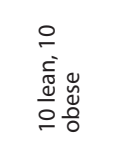 & 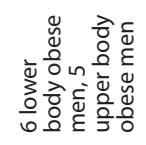 & & 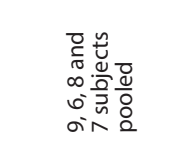 & & $\stackrel{\sim}{N}$ & 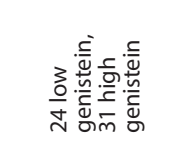 \\
\hline 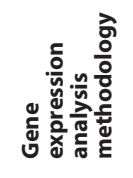 & 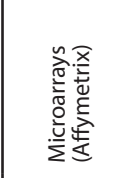 & 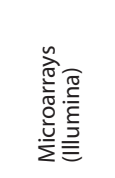 & 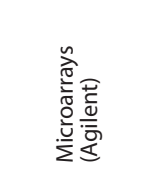 & & 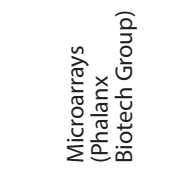 & & 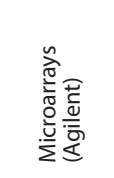 & 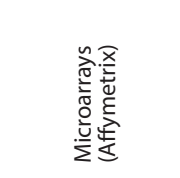 \\
\hline 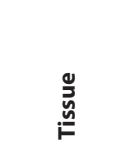 & $\sum_{0}^{\cup}$ & 号 & 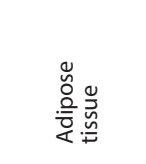 & & $\begin{array}{l}\frac{0}{0} \\
\frac{0}{0} \\
\frac{0}{0} \\
\frac{0}{3}\end{array}$ & & 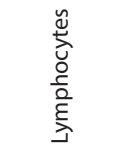 & 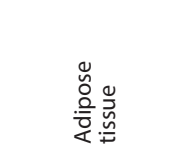 \\
\hline 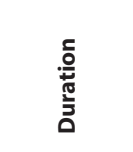 & 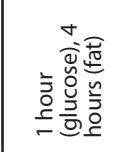 & 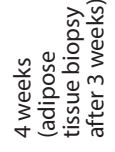 & 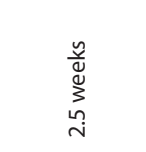 & & 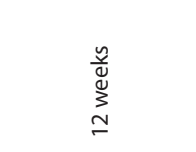 & & 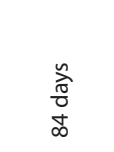 & 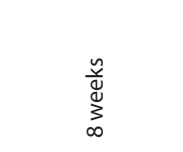 \\
\hline 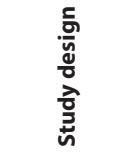 & 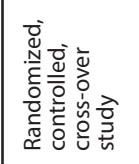 & 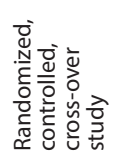 & 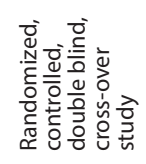 & & 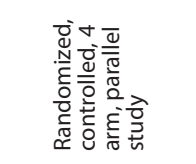 & & 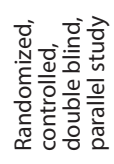 & 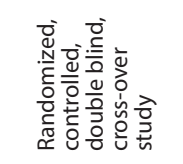 \\
\hline 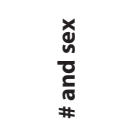 & 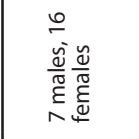 & $\begin{array}{l}\frac{\tilde{u}}{\pi} \\
\underset{m}{\tilde{n}}\end{array}$ & 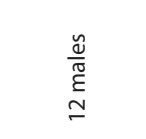 & & $\begin{array}{l}\frac{\tilde{\omega}}{\pi} \\
\tilde{\varepsilon} \\
\stackrel{\sim}{\sim}\end{array}$ & & 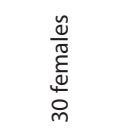 & 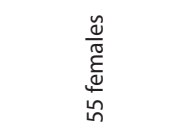 \\
\hline $\begin{array}{l}\frac{5}{0} \\
\frac{0}{0} \\
\frac{0}{2} \\
\frac{0}{2}\end{array}$ & 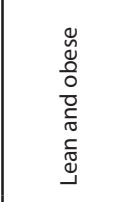 & 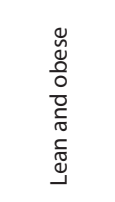 & 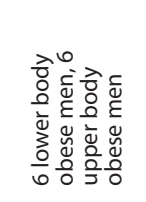 & & 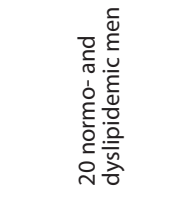 & & 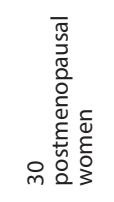 & 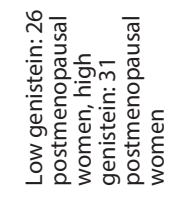 \\
\hline 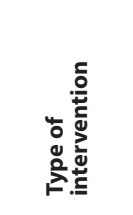 & 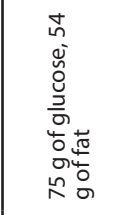 & 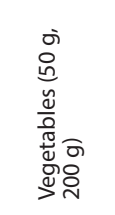 & 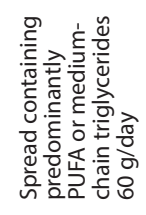 & 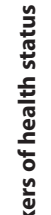 & 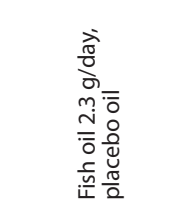 & : & 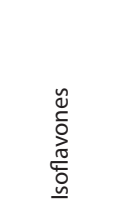 & 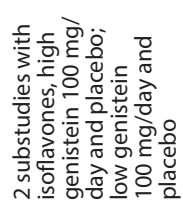 \\
\hline $\begin{array}{l}\text { 흘 } \\
\text { चे }\end{array}$ & 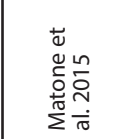 & 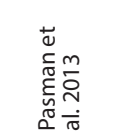 & 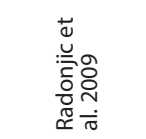 & 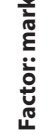 & 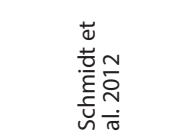 & 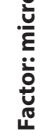 & 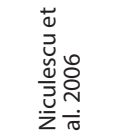 & 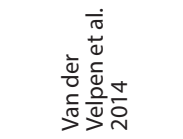 \\
\hline
\end{tabular}




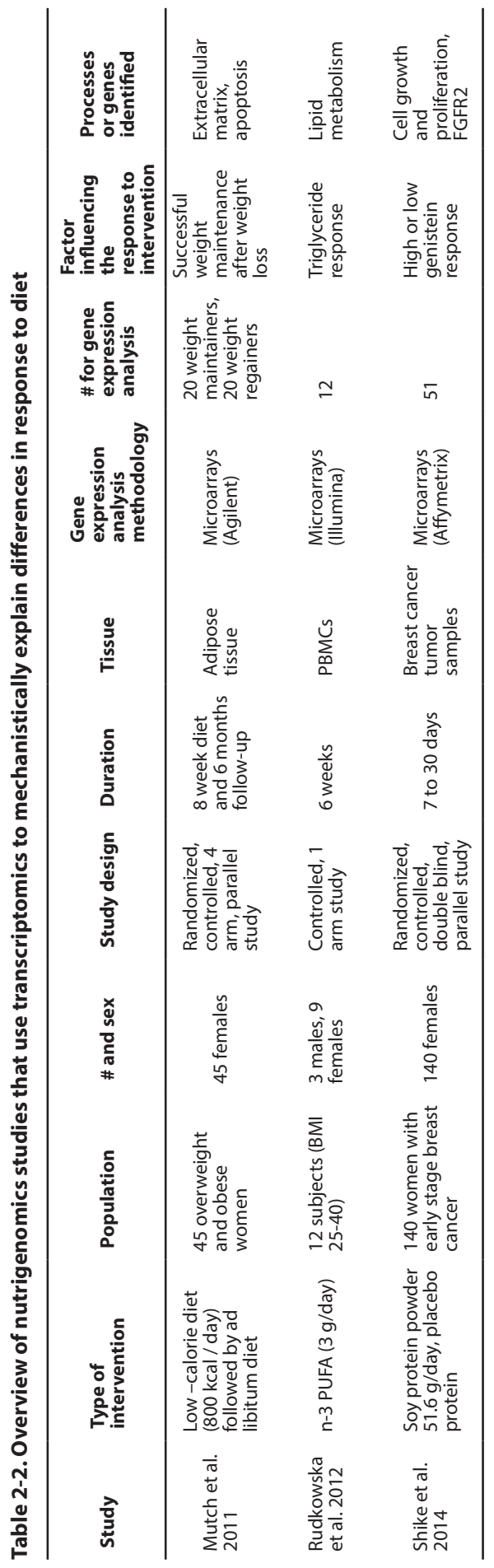




\section{Apolipoprotein E genotype status affects habitual human blood mononuclear cell gene expression and its response to a fish-oil intervention}

Juri Matualatupauw, Marijana Radonjic, Ondine van de Rest, Lisette de Groot, Marianne Geleijnse, Michael Müller and Lydia Afman

Mol Nutr Food Res. 2016 Jul;60(7):1649-60. 


\section{Abstract}

\section{Background}

People who carry the apolipoprotein E4 (APOE4) SNP have an increased risk of cardiovascular disease (CVD). Fish-oil supplementation may help in the prevention of CVD, though interindividual differences in the response to n-3 PUFAs have been observed. We aimed to assess the impact of APOE genotype on peripheral blood mononuclear cell (PBMC) whole genome gene expression at baseline and following a fish-oil intervention.

\section{Methods}

Participants received 6 months of fish-oil supplementation containing $1800 \mathrm{mg}$ of eicosapentaenoic acid and docosahexaenoic acid per day. APOE genotype and PBMC whole genome gene expression before and after supplementation were measured. We characterized the differences in gene expression profiles in carriers of APOE4 $(\mathrm{N}=8)$ compared to noncarriers $(\mathrm{N}=15)$.

\section{Results}

At baseline, 1320 genes were differentially expressed and the fish-oil supplementation differentially regulated 866 genes between APOE4 carriers and non-carriers. Gene set enrichment analysis showed that carriers had a higher gene expression of cholesterol biosynthesis and interferon (IFN) signaling pathways. Fish-oil supplementation reduced expression of IFN-related genes in carriers only.

\section{Conclusion}

The increased expression of IFN signaling and cholesterol biosynthesis pathways might explain part of the association between APOE4 and CVD. Fish-oil supplementation may particularly benefit APOE4 carriers by decreasing expression of IFN-related genes. 


\section{Introduction}

Apolipoprotein E (APOE) is part of several types of lipoproteins including VLDL, HDL and chylomicrons (1). Three major isoforms exist: APOE2, APOE3 and APOE4, which have allele frequencies of $6 \%, 15 \%$ and $78 \%$ respectively (2). Large meta-analyses have shown that carriers of APOE4 have a modestly increased risk of coronary heart disease $(3,4)$ and stroke (5) compared with individuals with the APOE3/APOE3 genotype, though the magnitude of this finding differs between studies and it is not always significant (4). Furthermore APOE4 is associated with increased levels of LDL cholesterol (4-6) and triglycerides (7) in the plasma.

For prevention of CVD, the European Society of Cardiology recommends two fatty fishcontaining meals a week (8). A meta-analysis of randomized controlled trials showed that supplementary n-3 polyunsaturated fatty acids (PUFAs) decreases the risk of CVD (9). However, this positive impact on CVD is not reported consistently; several studies find no association between n-3 PUFA supplementation and CVD risk (10). Furthermore, interindividual differences in the response to n-3 PUFAs have been observed. This inter-individual variability may, in part, be caused by genetic factors (11).

Several studies have shown that APOE genotype influences the plasma lipid response to fishoil supplementation. Minihane et al. 2000 (12) found a significant increase in total cholesterol and a trend toward a reduction in HDL-Cholesterol in APOE4 carriers relative to APOE3/ APOE3 subjects after fish-oil supplementation. In line with this, Olano-martin et al. 2010 (13) found increased total cholesterol concentrations in APOE4 carriers in response to 4 weeks docosahexaenoic acid (DHA) supplementation. Caslake et al 2008 (14) found the greatest decrease in plasma triglyceride concentration in APOE4 men after fish-oil supplementation.

We previously showed that 6 months fish-oil supplementation decreased gene expression of genes involved in inflammatory and atherogenic pathways in peripheral blood mononuclear cells (PBMCs) (15). PBMCs are a subpopulation of circulating immune cells mainly consisting of monocytes and lymphocytes. These circulating immune cells are important players in the pathogenesis of CVD, especially atherosclerosis, and, therefore, are a suitable target for studying inflammatory and CVD mechanisms (16). APOE genotype has been shown to affect immune cell response (17-19). For example, mice monocytes/macrophages showed an increased inflammatory response when transfected to produce human APOE4 compared to APOE3 (17). Moreover, signaling via the APOE receptors promotes conversion of macrophages from the pro-inflammatory M1 to the anti-inflammatory M2 phenotype (18), 
which may be decreased in APOE4 carriers because of the lower APOE concentrations that are observed with APOE4 (19). From these results, it is thought that the altered inflammatory response of APOE4-expressing monocytes/macrophages may contribute to the higher CVD risk observed in APOE4 carriers.

By measuring PBMC whole genome gene expression with microarrays and combining this with APOE genotyping, we are able to unbiasedly study genotype-specific phenotype effects.

In this study, we aimed to gain more insight in the pathways affected by APOE4 and the effect of fish-oil supplementation on these pathways by studying gene-diet interactions between APOE4 and fish-oil supplementation on whole genome gene expression. To do this, we conducted a secondary retrospective analysis according to APOE4 carrier status of the abovementioned study $(15,20)$. Firstly, we studied the effect of APOE4 on whole genome PBMC gene expression at baseline and, secondly, we studied how APOE4 influences the effects of 6 months of fish-oil supplementation on gene expression.

\section{Methods}

\section{Study design}

This study is a secondary retrospective analysis of a randomized, double-blind, placebocontrolled trial conducted by van de Rest et al. (2008) (20) that was originally designed to examine the effects of 6 months fish-oil supplementation on cognitive performance in 302 participants aged $\geq 65$ years. In this secondary analysis, we examine differences in whole genome gene expression between APOE4 carriers and APOE4 non-carriers before and following the fish-oil intervention. Microarray data was available in 23 subjects from the high dose fish-oil group (15), with RNA available in 92 participants. To validate findings of the microarray analyses, we performed targeted QPCR measurements in these 92 participants

Design and methods of the original study were described in detail previously (20). Briefly, participants were randomly allocated to receive a daily dose of fish-oil containing either 1800 mg or 400 mg eicosapentaenoic acid (EPA) and DHA, or a placebo oil (high-oleic sunflower oil (HOSF)) for 26 weeks. The high daily dose of fish oil provided $1093 \pm 17 \mathrm{mg}$ EPA and 847 $\pm 23 \mathrm{mg}$ DHA, and the low daily dose provided $226 \pm 3 \mathrm{mg}$ EPA and $176 \pm 4 \mathrm{mg}$ DHA. The oils were administered in 6 soft gelatin capsules daily, each of which contained $900 \mathrm{mg}$ oil and $2.7 \mathrm{mg}$ tocopherol as antioxidant (Banner Pharmacaps Europe BV, Tilburg, Netherlands). 
Participants were recruited according to the following exclusion criteria: current or recent ( $<4$ weeks) use of fish-oil supplements or intake of fish $>4$ times/week or $>800 \mathrm{mg}$ fish-oil/d from fish as estimated by using a fish-consumption questionnaire, serious liver disease, consumption of $>4$ glasses of alcohol-containing beverages per day, unable to participate as judged by the responsible medical physician, allergy to fish or fish-oil, swallowing problems, or participation in another clinical trial $<2$ mo before the start of the trial or at the same time. Cognitive exclusion criteria were also used and were described previously (20). Additionally, compliance with capsule use during a 2 -week placebo run-in period had to be $\geq 80 \%$ on the basis of self-report. All participants gave written informed consent to participate in the study and the study protocol was approved by the Medical Ethical Committee of Wageningen University, Wageningen, the Netherlands. The study was registered at clinicaltrials.gov as NCT00124852.

\section{Blood sampling and PBMC isolation}

Fasting venous blood samples were collected at baseline and after 26 weeks of intervention. Plasma free fatty acids and triglycerides were measured by gas-liquid chromatography, and C-reactive protein (CRP) concentrations were determined from measurements of high-sensitivity CRP (hsCRP). For PBMC isolation, $4 \mathrm{~mL}$ blood was collected into Becton Dickinson Vacutainer Cell Preparation Tubes with sodium citrate. PBMCs were isolated immediately after blood collection according to the manufacturer's instructions. For APOE genotyping, a second blood sample was collected into a 4.5-mL EDTA Vacutainer and stored at $-80^{\circ} \mathrm{C}$.

\section{APOE genotyping}

APOE genotyping was done by the PCR-based restriction fragment length polymorphism method and restriction enzyme digestion with HhaI (21). We retrospectively determined APOE genotype in 301 of the participants of the original study.

\section{RNA extraction and microarray}

RNA extraction and microarray methods were previously described by Bouwens et al. (15) and data can be found in Gene Expression Omnibus under accession number GSE12375. We reanalyzed the data using the current gene definitions (NuGOHs1a520180_Hs_ENTREZG MBNI custom CDF version 19.0.0) and grouped the samples based on the APOE genotyping results (Supplementary Table S3-1). 
Microarray analysis was performed on baseline samples and on samples after 26 weeks of intervention, using human whole-genome NuGO GeneChip arrays designed by the European Nutrigenomics Organization and manufactured by Affymetrix (Affymetrix Inc, Santa Clara, CA). Microarrays were analyzed using MADMAX (Management and Analysis Database for Multiplatform Microarray Experiments) (22). Expression values were normalized using the RMA (robust multichip average method) (23). Genes with normalized expression values $>20$ on at least 5 arrays were defined as expressed and selected for further analysis. Expression values were log2-transformed. LIMMA (24) was used to calculate P-values and false discovery rate (FDR) q-values for each gene using t-tests with Bayesian correction. At baseline, genes were defined as differentially expressed between APOE4 carriers and non-carriers if they had $\mathrm{P}$-values $<0.05$. The differences in response to fish-oil supplementation were calculated using the individual log ratios. Differences in genes expression changes between APOE4 carriers and non-carriers were defined as significantly different if they had P-values $<0.05$.

Pathway analysis and upstream transcription regulators analyses were performed using QIAGEN's Ingenuity Pathway Analysis (IPA, QIAGEN Redwood City, www.qiagen.com/ ingenuity). We also performed pathway analysis using gene set enrichment analysis (GSEA; http://www.broad.mit.edu/gsea) (25). Briefly, genes were ranked based on the t-statistic and analyzed for over- or underrepresentation in predefined gene sets. Gene sets were derived from Biocarta, KEGG, Reactome and Wikipathways pathway databases. Genesets with a false discovery rate $<0.1$ were considered significantly enriched (Supplementary Table S3-2 - S35).

\section{QPCR}

For qPCR, RNA was available of PBMCs of 92 participants of which 31 were carriers and 61 were non-carriers of APOE4. RNA was reverse transcribed using a cDNA synthesis kit (RevertAid First Strand cDNA Synthesis kit, Thermo Scientific, Leusden, the Netherlands) and analyzed by qPCR (SensiMix SYBR No-ROX, Bioline, London, UK) on a CFX384 RealTime System (C1000 Thermal Cycler, Biorad, Veenendaal, The Netherlands). qPCR data were normalized using the RPLP0 housekeeping gene. Genes for qPCR were selected from the differentially expressed pathways between APOE4 carriers and non-carriers. Genes needed to be differently expressed between APOE4 carriers and non-carriers and have an expression value above 200. Primers sequences are shown in Table 3-1.

\section{Statistics}


Statistical analysis of subject characteristics and qPCR findings was performed using IBM SPSS Statistics, Version 22.0.0.1. A two-sided P $<0.05$ was considered significant. Subject characteristics were reported as mean $\pm \mathrm{sd}$. Baseline characteristics of APOE4 carrier and non-carrier groups were compared using independent t-tests. QPCR results were analyzed using independent t-tests.

Power calculations were performed retrospectively for the HMGCS1 gene. Power was calculated to be $83 \%$, using the mean expression values and standard deviations as observed (218.7 and 184.8 for APOE4 carriers; and 28.2 and 23.5 for APOE4 non-carriers).

\section{Results}

\section{Participant characteristics}

The original study consisted of 302 participants. Here, we focused on a subgroup of participants who received $1800 \mathrm{mg}$ fish-oil and on whom PBMC whole genome gene expression microarray analysis was performed $(\mathrm{N}=23)$. APOE genotyping showed that 8 of the 23 participants were carriers of APOE4. Baseline characteristics of carriers and non-carriers are presented in Table 3-2. We found no differences in any parameters between the groups. Participants received daily fish-oil supplementation containing $1800 \mathrm{mg}$ of EPA and docosahexaenoic acid (DHA) for 6 months. We found no difference in the changes in any parameters between APOE4 carriers and non-carriers (Table 3-2).

\section{Baseline}

To determine the effects of APOE4 on gene expression, baseline expression profiles were compared between APOE4 carriers and non-carriers. Of the 13027 genes found to be expressed, 1320 genes were differentially expressed between carriers and non-carriers of the APOE4 SNP at $\mathrm{P}<0.05$ (Figure 3-1). No genes showed an FDR $\mathrm{q}<0.05$.

To gain further insight into the biological processes affected by APOE4, we performed pathway analysis using Ingenuity Pathway Analysis on the set of differentially expressed genes at baseline. Figure 3-2 shows the top 10 pathways that were enriched in the comparison of APOE4 carriers and non-carriers. Several of these pathways were related to either cholesterol biosynthesis or interferon (IFN) signaling. Furthermore, GSEA showed several gene sets related to cholesterol biosynthesis or IFN signaling as upregulated. (Supplementary Table S3-2). 
Further inspection of the function of the genes related to cholesterol biosynthesis, showed that many of these genes are encoding enzymes involved in de novo synthesis of cholesterol. These genes were found to be more highly expressed in APOE4 carriers than in non-carriers. Genes involved in cholesterol uptake (LDLR and CD36) and efflux (ABCA1, ABCG1 and SCARB1) showed no difference in expression in APOE4 carriers compared to non-carriers.

We observed a higher expression of genes involved in IFN signaling in carriers compared to non-carriers of APOE4. These genes included IFN (alpha, beta and omega) receptor 1 (IFNAR1) as well as the intracellular signaling molecules Signal Transducers and Activator of Transcription (STAT)1 and STAT2. Besides IFN signaling genes, we also found a higher expression of many IFN target genes in carriers of the APOE4 SNP compared to non-carriers (Table 3-3 and Figure 3).

To examine potential regulators of the observed gene expression differences between carriers and non-carriers, we performed Ingenuity upstream regulator analysis. IFNL1, IFNA2 and IFNG were identified as potential transcriptional regulators of the observed gene expression differences. These regulators and their targets are shown in Figure 3. Additionally, several other molecules that are involved in IFN signaling were identified as potential upstream regulators (Table 3-4).

\section{Fish-oil intervention effect}

To examine the effect of APOE4 on the gene expression response to fish-oil supplementation, we determined the genes that were differentially regulated in response to fish-oil supplementation in carriers compared to non-carriers of APOE4. We found that 866 genes showed a different change in expression caused by fish-oil supplementation between carriers and non-carriers at $\mathrm{P}<0.05$ (Figure 3-1). No genes showed an FDR $\mathrm{q}<0.05$.

Figure 3-4 shows the enriched pathways when comparing the response to fish-oil supplementation between carriers and non-carriers of APOE4. The top 2 pathways are both related to IFN signaling. Furthermore, GSEA showed several gene sets related to IFN signaling as downregulated when comparing the response to fish-oil supplementation between carriers and non-carriers of APOE4 (Supplementary Table S3-5).

In carriers of the APOE4 allele, genes in these IFN signaling pathways were found to be mostly downregulated, whereas non-carriers mostly showed an upregulation in response to n-3 PUFA supplementation when comparing gene expression after supplementation to expression at baseline. 
To examine potential regulators of the differentially expressed genes, we performed Ingenuity upstream regulator analysis. This revealed many IFN-related genes as potential upstream transcriptional regulators, including IFNL1, IFNA2 and IFNG (Table 3-5). The genes regulated by these cytokines were found to be largely downregulated in APOE4 carriers and largely upregulated in non-carriers, as shown in Figure 3-5.

Comparison of gene expression profiles after 6 months fish-oil supplementation between carriers and non-carriers of APOE4 showed that both cholesterol biosynthesis as well as IFN signaling pathways were no longer enriched in the comparison between carriers and noncarriers of APOE4 (data not shown).

\section{QPCR validation of the microarray findings}

QPCR was used to determine the expression of five genes selected from the cholesterol biosynthesis and IFN signaling pathways in all available RNA samples. We determined gene expression of HMGCR, HMGCS1, IFITM1, STAT1 and TAP1 in a total of 92 participants, of which 31 were APOE4 carriers and 61 were APOE4 non-carriers. We found that expression of HMGCS1 and STAT1 was significantly higher in APOE4 carriers compared to non-carriers at baseline (Figure 3-6). Mean expression of HMGCR, IFITM1 and TAP1 was higher in APOE4 carriers compared to non-carriers, though these differences were not significant.

\section{Discussion}

This study examined the effect of APOE4 on whole genome gene expression in PBMCs at baseline and in response to fish-oil supplementation. Expression of genes involved in cholesterol biosynthesis as well as IFN signaling and IFN target genes was found to be higher in APOE4 carriers compared to non-carriers. Interestingly, 6 months of fish-oil supplementation decreased IFN-related gene expression in APOE4 carriers.

The increased expression of genes in the IFN signaling pathway and IFN-regulated genes in PBMCs of APOE4 carriers at baseline may point towards a systemic pro-inflammatory state. Consistent with our findings, APOE4 has been linked to increased systemic inflammation as carriers of the APOE4 allele have been shown to have higher plasma levels of the proinflammatory cytokines IL-8 and TNF- $\alpha$ (26), and lower levels of the anti-inflammatory cytokine IL-10 (27). Furthermore, IFNG produced by T-lymphocytes is highly expressed in atherosclerotic lesions and affects many processes involved in the pathogenesis of 
atherosclerosis (28). Increased expression of genes in the IFN signaling pathway as well as increased expression of IFN targets in PBMCs of APOE4 carriers may unfavorably prime these cells and may increase the risk of atherosclerosis. The question remains whether the observed increase in IFN-related gene expression is caused by APOE4 directly, or whether it is a consequence of indirect factors that are affected by APOE4.

Literature on the role of fish-oil or n-3 PUFAs on IFN pathways and IFN-regulated genes is scarce. It is possible that previous studies that did not take APOE genotype into account found no effects, as we show in the current study that IFN signaling-related gene expression is either upregulated or downregulated dependent on APOE genotype. We identified one mouse study, in which a high intake of n-3 PUFAs was shown to lead to diminished STAT1 phosphorylation after ex-vivo stimulation of immune cells with IFN-gamma (29). A similar mechanism could be present in our APOE4 carriers, though, to our knowledge, no studies exist that have examined the role of APOE genotype in this context. One relatively small study in 35 Alzheimer's disease patients examined the interaction between APOE genotype and 6 months of fish-oil supplementation on several inflammatory cytokines in the plasma, but found no effects of APOE genotype (30).

In contrast to APOE4 carriers, non-carriers showed an upregulation of IFN pathways. Previously, we examined the effects of fish-oil supplementation in this population and found anti-inflammatory and anti-atherogenic gene expression changes when not taking APOE genotype into account (15). These changes were not found in genes related to IFN signaling pathways. This indicates that fish-oil supplementation may have pro-inflammatory as well as anti-inflammatory effects in APOE4 non-carriers. Further research is required to fully understand the APOE genotype-specific effects of fish-oil supplementation on inflammatory signaling.

APOE4 is associated with an increased risk of CVD (4). Partly, this is thought to be mediated by increased LDL-cholesterol and triglyceride plasma concentrations in APOE4 carriers (31). In the full study group of 301 participants, we also observed significantly higher LDLcholesterol levels in the plasma of APOE4 carriers compared to non-carriers. Our microarray data point towards an increase in cholesterol synthesis as reflected by a higher expression of several genes encoding enzymes involved in the synthesis of cholesterol, including HMGCoA reductase (HMGCR), the enzyme catalyzing the rate-limiting step in the synthesis of cholesterol (32). Expression of this gene and other genes involved in cholesterol biosynthesis is known to be controlled by the SREBP-2 transcription factor through a negative feedback 
system (33). Therefore, the higher expression of cholesterol biosynthesis genes in APOE4 carriers might suggests a lower intracellular cholesterol concentration and, consequently, a reduced activity of SREBP-2. However, the SREBP-2 gene itself was not differentially expressed between APOE4 carriers and non-carriers and, based on the increased LDLcholesterol concentrations in APOE4 carriers that we observed in the full study group of 301 participants and is consistently reported in literature, one might expect increased intracellular cholesterol levels. Whether cholesterol levels are actually increased in the cell requires further investigation.

The role of increased cholesterol synthesis in monocytes remains unclear. It may promote cholesterol accumulation in these cells, which is an important first step in foam cell formation and may, therefore, potentially be related to the increased CVD risk in APOE4 carriers. Additionally, in a study by Gerdes et al. (2000) (34), treatment with simvastatin, an inhibitor of the HMGCR protein, abolishes the APOE4-related 2-fold increase in mortality after a previous myocardial infarction. This study indicates that persons carrying a copy of the APOE4 allele are especially sensitive to cholesterol synthesis altering interventions. HMGCR gene expression in PBMCs has been shown to closely parallel liver gene expression (35). If the higher expression of this gene and other SREBP-2-regulated cholesterol biosynthesis genes is present in liver as well, it may possibly explain why APOE4 carriers are especially sensitive to cholesterol synthesis altering interventions with respect to CVD risk.

In this study, we describe changes in two pathways that may potentially contribute to the increased CVD risk that is observed in carriers of APOE4: (1) increased expression of IFN signaling and IFN target genes, and (2) increased expression of cholesterol biosynthesis genes. Several studies have examined a possible link between inflammation and cholesterol synthesis. Chen et al. (36) found that inflammatory stress causes intracellular cholesterol accumulation, and increases HMGCR gene and protein expression via the SREBP-2 pathway in mice both in vitro as well as in vivo. Furthermore, Zhao et al. (37) induced inflammatory stress in mice and in a human cell line and found increased cholesterol synthesis and SREBP-2 and HMGCR gene and protein expression. Taking these findings and our gene expression results together, it might be possible that increased inflammatory stress, as indicated by increased IFN signaling in APOE4 carriers may have affected cholesterol biosynthesis gene expression. Further research is required, however, to explore the role of inflammatory stress and cholesterol biosynthesis as potential mechanisms for the increased CVD risk in APOE4 carriers as well as the relationship between these processes. 
Although the expression of genes in the cholesterol biosynthesis pathway was significantly higher in APOE4 carriers compared to non-carriers, we did not observe a significant response to fish-oil supplementation in either group. When comparing carriers and non-carriers after supplementation the cholesterol biosynthesis pathway is no longer significantly differently expressed, suggesting that fish-oil might have affected gene expression of these genes to some extent. Possibly, these changes may have gone undetected due to the relatively low number of participants, especially in the APOE4 group.

A limitation of this study is that, due to the fact that it was not originally designed to answer the current research question, the groups are relatively small and not of the same size. We performed power calculations, which showed that we had sufficient power for the microarray analyses. Distinct differences in gene expression between carriers and non-carriers of the APOE4 allele, both at baseline as well as in the response to a 6-month fish-oil intervention were observed. Furthermore, several of the differences at baseline were confirmed in a larger group using qPCR. For our microarray analyses, we used a relatively high P-value cut-off of 0.05. Using FDR q-values for selecting the differentially expressed genes did not yield results in several comparisons. For this reason, we focused our analyses mainly on the pathway level. In this study, we opted not to include to placebo group in the analysis (15). Firstly, because our study is focused on the differences between APOE4 carriers and non-carriers in the response to fish-oil. Secondly, adding this group, would further complicate this already quite complicated analysis and, as a consequence, reduce the readability of the paper.

The opposite effect of fish-oil supplementation in APOE4 carriers and non-carriers that we found, can partially explain the heterogeneity that is seen in the responses to fish-oil supplementation on gene expression profiles and illustrates the importance of research on diet-gene interactions and research into personalized nutrition in general.

Our findings related to IFN signaling and cholesterol biosynthesis might explain part of the association between APOE4 and the increased risk of CVD that is observed in carriers of this allele. Additionally, with respect to our IFN signaling gene expression results we hypothesize that fish-oil supplementation may particularly benefit APOE4 carriers.

\section{References}

1. Mahley RW, Innerarity TL. Lipoprotein receptors and cholesterol homeostasis. Biochimica et biophysica acta. 1983;737(2):197-222. 
2. Eisenberg DT, Kuzawa CW, Hayes MG. Worldwide allele frequencies of the human apolipoprotein E gene: climate, local adaptations, and evolutionary history. Am J Phys Anthropol. 2010;143(1):100-11.

3. Song Y, Stampfer MJ, Liu S. Meta-analysis: apolipoprotein E genotypes and risk for coronary heart disease. Ann Intern Med. 2004;141(2):137-47.

4. Bennet AM, Di Angelantonio E, Ye Z, Wensley F, Dahlin A, Ahlbom A, et al. Association of apolipoprotein E genotypes with lipid levels and coronary risk. JAMA. 2007;298(11):1300-11.

5. Khan TA, Shah T, Prieto D, Zhang W, Price J, Fowkes GR, et al. Apolipoprotein E genotype, cardiovascular biomarkers and risk of stroke: systematic review and meta-analysis of 14,015 stroke cases and pooled analysis of primary biomarker data from up to 60,883 individuals. Int J Epidemiol. 2013;42(2):475-92.

6. Davignon J, Gregg RE, Sing CF. Apolipoprotein E polymorphism and atherosclerosis. Arteriosclerosis. 1988;8(1):1-21.

7. Dallongeville J, Lussier-Cacan S, Davignon J. Modulation of plasma triglyceride levels by apoE phenotype: a meta-analysis. Journal of lipid research. 1992;33(4):447-54.

8. Perk J, De Backer G, Gohlke H, Graham I, Reiner Z, Verschuren M, et al. European Guidelines on cardiovascular disease prevention in clinical practice (version 2012). The Fifth Joint Task Force of the European Society of Cardiology and Other Societies on Cardiovascular Disease Prevention in Clinical Practice (constituted by representatives of nine societies and by invited experts). Eur Heart J. 2012;33(13):1635-701.

9. Bucher HC, Hengstler P, Schindler C, Meier G. N-3 polyunsaturated fatty acids in coronary heart disease: a meta-analysis of randomized controlled trials. Am J Med. 2002;112(4):298-304.

10. Rizos EC, Ntzani EE, Bika E, Kostapanos MS, Elisaf MS. Association between omega-3 fatty acid supplementation and risk of major cardiovascular disease events: a systematic review and meta-analysis. JAMA. 2012;308(10):1024-33.

11. Madden J, Williams CM, Calder PC, Lietz G, Miles EA, Cordell H, et al. The impact of common gene variants on the response of biomarkers of cardiovascular disease (CVD) risk to increased fish oil fatty acids intakes. Annu Rev Nutr. 2011;31:203-34.

12. Minihane AM, Khan S, Leigh-Firbank EC, Talmud P, Wright JW, Murphy MC, et al. ApoE polymorphism and fish oil supplementation in subjects with an atherogenic lipoprotein phenotype. Arterioscler Thromb Vasc Biol. 2000;20(8):1990-7.

13. Olano-Martin E, Anil E, Caslake MJ, Packard CJ, Bedford D, Stewart G, et al. Contribution of apolipoprotein E genotype and docosahexaenoic acid to the LDL-cholesterol response to fish oil. Atherosclerosis. 2010;209(1):104-10.

14. Caslake MJ, Miles EA, Kofler BM, Lietz G, Curtis P, Armah CK, et al. Effect of sex and genotype on cardiovascular biomarker response to fish oils: the FINGEN Study. The American journal of clinical nutrition. 2008;88(3):61829.

15. Bouwens M, van de Rest O, Dellschaft N, Bromhaar MG, de Groot LC, Geleijnse JM, et al. Fish-oil supplementation induces antiinflammatory gene expression profiles in human blood mononuclear cells. The American journal of clinical nutrition. 2009;90(2):415-24.

16. de Mello VD, Kolehmanien M, Schwab U, Pulkkinen L, Uusitupa M. Gene expression of peripheral blood mononuclear cells as a tool in dietary intervention studies: What do we know so far? Mol Nutr Food Res. 2012;56(7):1160-72.

17. Jofre-Monseny L, Loboda A, Wagner AE, Huebbe P, Boesch-Saadatmandi C, Jozkowicz A, et al. Effects of apoE genotype on macrophage inflammation and heme oxygenase-1 expression. Biochemical and biophysical research communications. 2007;357(1):319-24.

18. Baitsch D, Bock HH, Engel T, Telgmann R, Muller-Tidow C, Varga G, et al. Apolipoprotein E induces antiinflammatory phenotype in macrophages. Arterioscler Thromb Vasc Biol. 2011;31(5):1160-8. 
19. Martinez-Morillo E, Hansson O, Atagi Y, Bu G, Minthon L, Diamandis EP, et al. Total apolipoprotein E levels and specific isoform composition in cerebrospinal fluid and plasma from Alzheimer's disease patients and controls. Acta neuropathologica. 2014;127(5):633-43.

20. van de Rest O, Geleijnse JM, Kok FJ, van Staveren WA, Dullemeijer C, Olderikkert MG, et al. Effect of fish oil on cognitive performance in older subjects: a randomized, controlled trial. Neurology. 2008;71(6):430-8.

21. Hixson JE, Vernier DT. Restriction isotyping of human apolipoprotein E by gene amplification and cleavage with HhaI. J Lipid Res. 1990;31(3):545-8.

22. Lin K, Kools H, de Groot PJ, Gavai AK, Basnet RK, Cheng F, et al. MADMAX - Management and analysis database for multiple omics experiments. Journal of integrative bioinformatics. 2011;8(2):160.

23. Irizarry RA, Bolstad BM, Collin F, Cope LM, Hobbs B, Speed TP. Summaries of Affymetrix GeneChip probe level data. Nucleic Acids Res. 2003;31(4):e15.

24. Gentleman R, Carey V, Huber W, Irizarry R, Dudoit S, Smyth GK. limma: Linear Models for Microarray Data. Bioinformatics and Computational Biology Solutions Using R and Bioconductor. Statistics for Biology and Health: Springer New York; 2005. p. 397-420.

25. Subramanian A, Tamayo P, Mootha VK, Mukherjee S, Ebert BL, Gillette MA, et al. Gene set enrichment analysis: a knowledge-based approach for interpreting genome-wide expression profiles. Proceedings of the National Academy of Sciences of the United States of America. 2005;102(43):15545-50.

26. Drabe N, Zund G, Grunenfelder J, Sprenger M, Hoerstrup SP, Bestmann L, et al. Genetic predisposition in patients undergoing cardiopulmonary bypass surgery is associated with an increase of inflammatory cytokines. Eur J Cardiothorac Surg. 2001;20(3):609-13.

27. Tziakas DN, Chalikias GK, Antonoglou CO, Veletza S, Tentes IK, Kortsaris AX, et al. Apolipoprotein E genotype and circulating interleukin-10 levels in patients with stable and unstable coronary artery disease. J Am Coll Cardiol. 2006;48(12):2471-81.

28. McLaren JE, Ramji DP. Interferon gamma: a master regulator of atherosclerosis. Cytokine \& growth factor reviews. 2009;20(2):125-35.

29. Irons R, Fritsche KL. Omega-3 polyunsaturated fatty acids impair in vivo interferon- gamma responsiveness via diminished receptor signaling. The Journal of infectious diseases. 2005;191(3):481-6.

30. Freund-Levi Y, Hjorth E, Lindberg C, Cederholm T, Faxen-Irving G, Vedin I, et al. Effects of omega-3 fatty acids on inflammatory markers in cerebrospinal fluid and plasma in Alzheimer's disease: the OmegAD study. Dement Geriatr Cogn Disord. 2009;27(5):481-90.

31. Minihane AM, Jofre-Monseny L, Olano-Martin E, Rimbach G. ApoE genotype, cardiovascular risk and responsiveness to dietary fat manipulation. Proc Nutr Soc. 2007;66(2):183-97.

32. Goldstein JL, Brown MS. Regulation of the mevalonate pathway. Nature. 1990;343(6257):425-30.

33. Horton JD, Goldstein JL, Brown MS. SREBPs: activators of the complete program of cholesterol and fatty acid synthesis in the liver. J Clin Invest. 2002;109(9):1125-31.

34. Gerdes LU, Gerdes C, Kervinen K, Savolainen M, Klausen IC, Hansen PS, et al. The apolipoprotein epsilon4 allele determines prognosis and the effect on prognosis of simvastatin in survivors of myocardial infarction : a substudy of the Scandinavian simvastatin survival study. Circulation. 2000;101(12):1366-71.

35. Powell EE, Kroon PA. Low density lipoprotein receptor and 3-hydroxy-3-methylglutaryl coenzyme A reductase gene expression in human mononuclear leukocytes is regulated coordinately and parallels gene expression in human liver. J Clin Invest. 1994;93(5):2168-74.

36. Chen $\mathrm{Y}, \mathrm{Ku} \mathrm{H}$, Zhao L, Wheeler DC, Li LC, Li Q, et al. Inflammatory stress induces statin resistance by disrupting 3-hydroxy-3-methylglutaryl-CoA reductase feedback regulation. Arterioscler Thromb Vasc Biol. 2014;34(2):365-76.

37. Zhao L, Chen Y, Tang R, Chen Y, Li Q, Gong J, et al. Inflammatory stress exacerbates hepatic cholesterol 
APOE4 and fish-oil

accumulation via increasing cholesterol uptake and de novo synthesis. Journal of gastroenterology and hepatology. 2011;26(5):875-83. 


\section{Tables}

Table 3-1. Primer sequences used to quantify gene expression by qPCR

\begin{tabular}{lll}
\hline Gene & Forward primer & Reverse Primer \\
\hline HMGCR & TGATTGACCTTTCCAGAGCAAG & CTAAAATTGCCATTCCACGAGC \\
HMGCS1 & CAGAAGAACTTACGCTCGGC & TCTTGGCAGGGCTTGGAATA \\
IFITM1 & AGGGACAGGAAGATGGTTGG & AATCAGGGCCCAGATGTTCA \\
RPLP0 & CAGATTGGCTACCCAACTGTT & GGGAAGGTGTAATCCGTCTCC \\
STAT1 & GAGAGTCTGCAGCAAGTTCG & GGAAAAGACTGAAGGTGCGG \\
TAP1 & ATTTGAGTACCTGGACCGCA & GCCCCTGTAGCACTAAGACA \\
\hline
\end{tabular}

Table 3-2. Baseline characteristics of the 23 participants of the high fish-oil supplementation group in which PBMC gene expression microarrays were performed. ${ }^{a}$

\begin{tabular}{lll}
\hline & APOE4 carriers (N=8) & APOE4 non-carriers (N=15) \\
\hline Age & $69 \pm 3$ & $69 \pm 3$ \\
M/F & $5 / 3$ & $10 / 5$ \\
M/F \% & $62 / 38$ & $67 / 33$ \\
Heigth (cm) & $170 \pm 8$ & $173 \pm 6$ \\
Weight (kg) & $77 \pm 7$ & $78 \pm 14$ \\
BMI (kg/m2) & $27.1 \pm 3.0$ & $26.1 \pm 3.4$ \\
Waist circumference (cm) & $96 \pm 6$ & $96 \pm 14$ \\
Total cholesterol (mmol/l) & $6.0 \pm 0.6$ & $5.4 \pm 0.9$ \\
Triglycerides (mmol/l) & $1.2 \pm 0.2$ & $0.9 \pm 0.4$ \\
LDL-cholesterol (mmol/l) & $4.1 \pm 0.5$ & $3.5 \pm 0.8$ \\
HDL-cholesterol (mmol/l) & $1.5 \pm 0.4$ & $1.5 \pm 0.3$ \\
hsCRP (mg/L)b & $2.0 \pm 1.9$ & $3.3 \pm 3.2$ \\
\hline
\end{tabular}

a Data presented as mean $\pm \mathrm{sd}$. Baseline values were not significantly different between carriers and non-carriers at $\mathrm{P}<0.05$.

${ }^{\mathrm{b}}$ hsCRP, high sensitivity C-reactive protein. 
Table 3-3. Baseline comparison of APOE4 carriers and non-carriers of genes involved in cholesterol biosynthesis, IFN signaling and IFN targets. a

\begin{tabular}{|c|c|c|c|c|}
\hline Gene & $\begin{array}{l}\text { Entrez } \\
\text { ID }\end{array}$ & Description & P-value ${ }^{b}$ & $\begin{array}{l}\text { Fold } \\
\text { chan }\end{array}$ \\
\hline \multicolumn{5}{|c|}{ Cholesterol synthesis } \\
\hline DHCR24 & 1718 & 24-dehydrocholesterol reductase & 0.02 & 1.24 \\
\hline GGPS1 & 9453 & geranylgeranyl diphosphate synthase 1 & 0.05 & 1.21 \\
\hline HADHA & 3030 & $\begin{array}{l}\text { hydroxyacyl-CoA dehydrogenase/3-ketoacyl-CoA thiolase/ } \\
\text { enoyl-CoA hydratase (trifunctional protein), alpha subunit }\end{array}$ & $<0.01$ & -1.12 \\
\hline HADHB & 3032 & $\begin{array}{l}\text { hydroxyacyl-CoA dehydrogenase/3-ketoacyl-CoA thiolase/ } \\
\text { enoyl-CoA hydratase (trifunctional protein), beta subunit }\end{array}$ & 0.04 & -1.08 \\
\hline HMGCR & 3156 & 3-hydroxy-3-methylglutaryl-CoA reductase & 0.02 & 1.24 \\
\hline HMGCS1 & 3157 & 3-hydroxy-3-methylglutaryl-CoA synthase 1 (soluble) & $<0.01$ & 1.18 \\
\hline IDI1 & 3422 & isopentenyl-diphosphate delta isomerase 1 & $<0.01$ & 1.14 \\
\hline
\end{tabular}

\section{IFN signaling}

$\begin{array}{lcllr}\text { IFNAR1 } & 3454 & \text { interferon (alpha, beta and omega) receptor 1 } & <0.01 & 1.26 \\ \text { IRF9 } & 10379 & \text { interferon regulatory factor } 9 & <0.01 & 1.26 \\ \text { MX1 } & 4599 & \text { MX dynamin-like GTPase 1 } & 0.02 & 1.73 \\ \text { PSMB8 } & 5696 & \text { proteasome (prosome, macropain) subunit, beta type, } 8 & <0.01 & 1.13\end{array}$

\section{IFN targets}

$\begin{array}{lcllc}\text { IFI35 } & 3430 & \text { interferon-induced protein 35 } & 0.01 & 1.33 \\ \text { IFIT1 } & 3434 & \text { interferon-induced protein with tetratricopeptide repeats } 1 & <0.01 & 2.54 \\ \text { IFIT3 } & 3437 & \text { interferon-induced protein with tetratricopeptide repeats 3 } & 0.01 & 2.01 \\ \text { IFITM1 } & 8519 & \text { interferon induced transmembrane protein 1 } & <0.01 & 1.17 \\ \text { IFNAR1 } & 3454 & \text { interferon (alpha, beta and omega) receptor 1 } & <0.01 & 1.26 \\ \text { IRF9 } & 10379 & \text { interferon regulatory factor 9 } & <0.01 & 1.26 \\ \text { MX1 } & 4599 & \text { MX dynamin-like GTPase 1 } & 0.02 & 1.73\end{array}$

a Genes selection is based on Ingenuity canonical pathways.

b P-values and fold changes for the comparison between APOE4 carriers and non-carriers are shown. P-values were considered significant when $\mathrm{P}<0.05$. 
Table 3-4. Top 10 potential upstream regulators ${ }^{\mathrm{a}}$ explaining the baseline differences in gene expression between APOE4 carriers and non-carriers. ${ }^{b}$

\begin{tabular}{llllll}
\hline $\begin{array}{l}\text { Upstream } \\
\text { Regulator }\end{array}$ & Fold Change & Molecule Type & $\begin{array}{l}\text { Predicted } \\
\text { Activation } \\
\text { State }\end{array}$ & $\begin{array}{l}\text { Activation } \\
\text { z-score }\end{array}$ & $\begin{array}{l}\text { P-value of } \\
\text { overlap }\end{array}$ \\
\hline PRL & -1.17 & cytokine & Activated & 6.76 & $3.77 \mathrm{E}-33$ \\
IFNL1 & 1.02 & cytokine & Activated & 6.21 & $2.14 \mathrm{E}-29$ \\
IFNA2 & -1.03 & cytokine & Activated & 6.17 & $3.06 \mathrm{E}-23$ \\
MAPK1 & -1.02 & kinase & Inhibited & -6.13 & $2.13 \mathrm{E}-18$ \\
CNOT7 & 1.03 & $\begin{array}{l}\text { transcription } \\
\text { regulator }\end{array}$ & Inhibited & -2.43 & $1.31 \mathrm{E}-15$ \\
IL1RN & 1.13 & cytokine & Inhibited & -4.64 & $1.89 \mathrm{E}-15$ \\
IFNG & 1.32 & cytokine & Activated & 5.45 & $5.33 \mathrm{E}-12$ \\
EIF2AK2 & 1.49 & kinase & Activated & 3.75 & $1.92 \mathrm{E}-09$ \\
Interferon alpha & & group & Activated & 4.01 & $2.90 \mathrm{E}-08$ \\
IFNB1 & & cytokine & Activated & 3.44 & $1.86 \mathrm{E}-06$ \\
\hline
\end{tabular}

a Ingenuity upstream regulator analysis identifies transcriptional regulators that can potentially explain the observed changes in gene expression.

${ }^{\mathrm{b}}$ The 10 regulators with the lowest $P$-value and a significant $Z$ score are shown. Fold change for the upstream regulator is shown if significantly differentially expressed. $Z$ scores predict the activation state of the regulator and are based on the gene expression of its downstream genes. Upstream regulators with $z$ scores $>2$ are considered activated and regulators with $z$ scores $<2$ are considered inhibited. P-values of overlap are calculated using Fisher's exact test. 


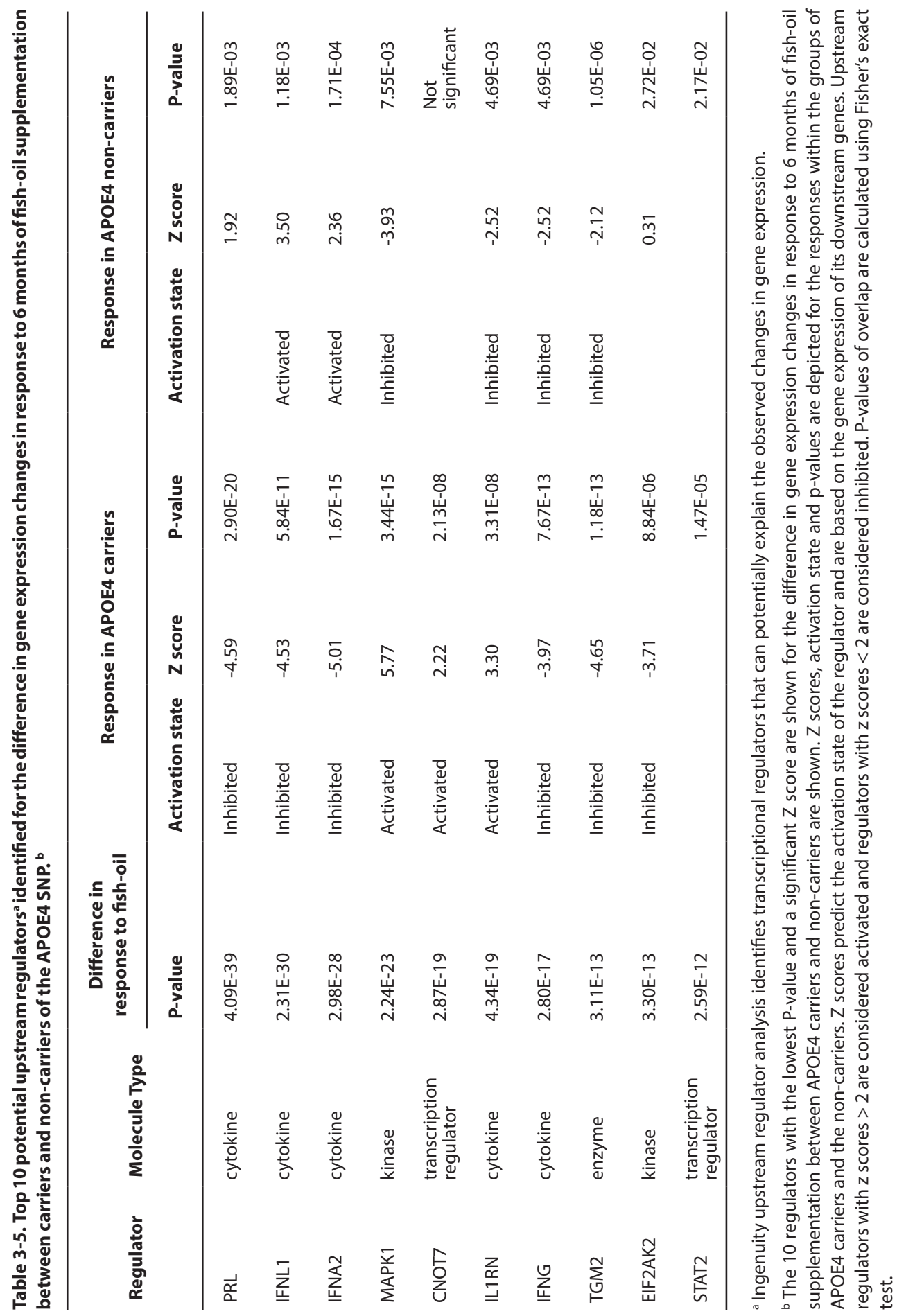




\section{Figures}

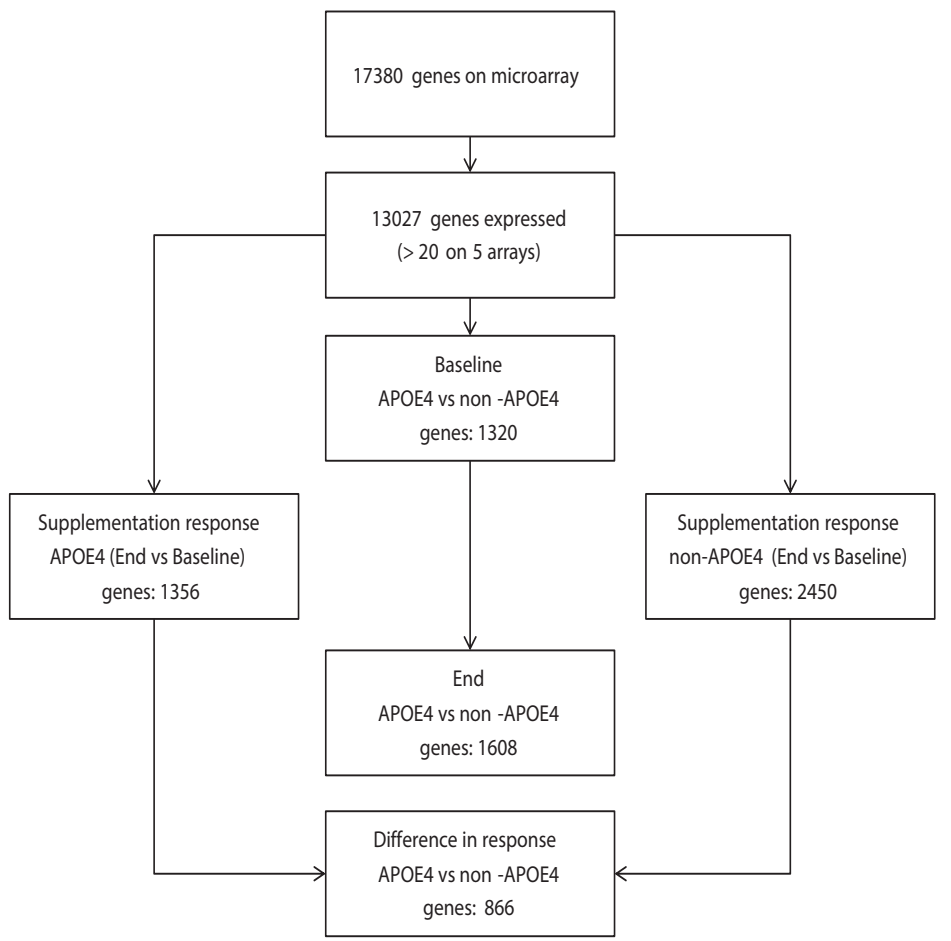

Figure 3-1. Flow chart of gene selection methods and number of significantly differentially expressed genes.

Genes were found to be significantly different if $\mathrm{P}<0.05$ 


\begin{tabular}{lccc}
\hline Pathway & P value & \multicolumn{1}{c}{ Total genes } & Up Down \\
\hline $\begin{array}{l}\text { Activation of IRF by Cytosolic Pattern Recognition } \\
\text { Receptors }\end{array}$ & $8.48 \mathrm{E}-08$ & 60 & 181 \\
$\begin{array}{l}\text { Interferon Signaling } \\
\text { Role of RIG1-like Receptors in Antiviral Innate }\end{array}$ & $3.64 \mathrm{E}-05$ & 34 & 110 \\
Immunity & $4.97 \mathrm{E}-05$ & 41 & 111 \\
$\begin{array}{l}\text { Death Receptor Signaling } \\
\text { Superpathway of Cholesterol Biosynthesis }\end{array}$ & $1.16 \mathrm{E}-05$ & 91 & 172 \\
$\begin{array}{l}\text { Protein Ubiquitination Pathway } \\
\begin{array}{l}\text { Role of PKR in Interferon Induction and Antiviral } \\
\text { Response }\end{array}\end{array}$ & $8.4 .17 \mathrm{E}-04$ & 27 & 72 \\
$\begin{array}{l}\text { Superpathway of Geranylgeranyldiphosphate } \\
\text { Biosynthesis I (via Mevalonate) }\end{array}$ & $9.47 \mathrm{E}-04$ & 254 & 297 \\
Mevalonate Pathway I & $1.49 \mathrm{E}-03$ & 60 & 42 \\
\begin{tabular}{l} 
PPAR Signaling \\
\hline
\end{tabular} & $1.64 \mathrm{E}-03$ & 12 & 32 \\
\hline
\end{tabular}

Figure 3-2. Top 10 differentially expressed pathways at baseline between APOE4 carriers and noncarriers.

Top 10 was based on the lowest P-value. Total genes: total number of genes in the pathway, up (red): number of genes with significantly higher expression in APOE4 carriers compared to non-carriers, down (green): number of genes with significantly lower expression in APOE4 carriers compared to non-carriers. 


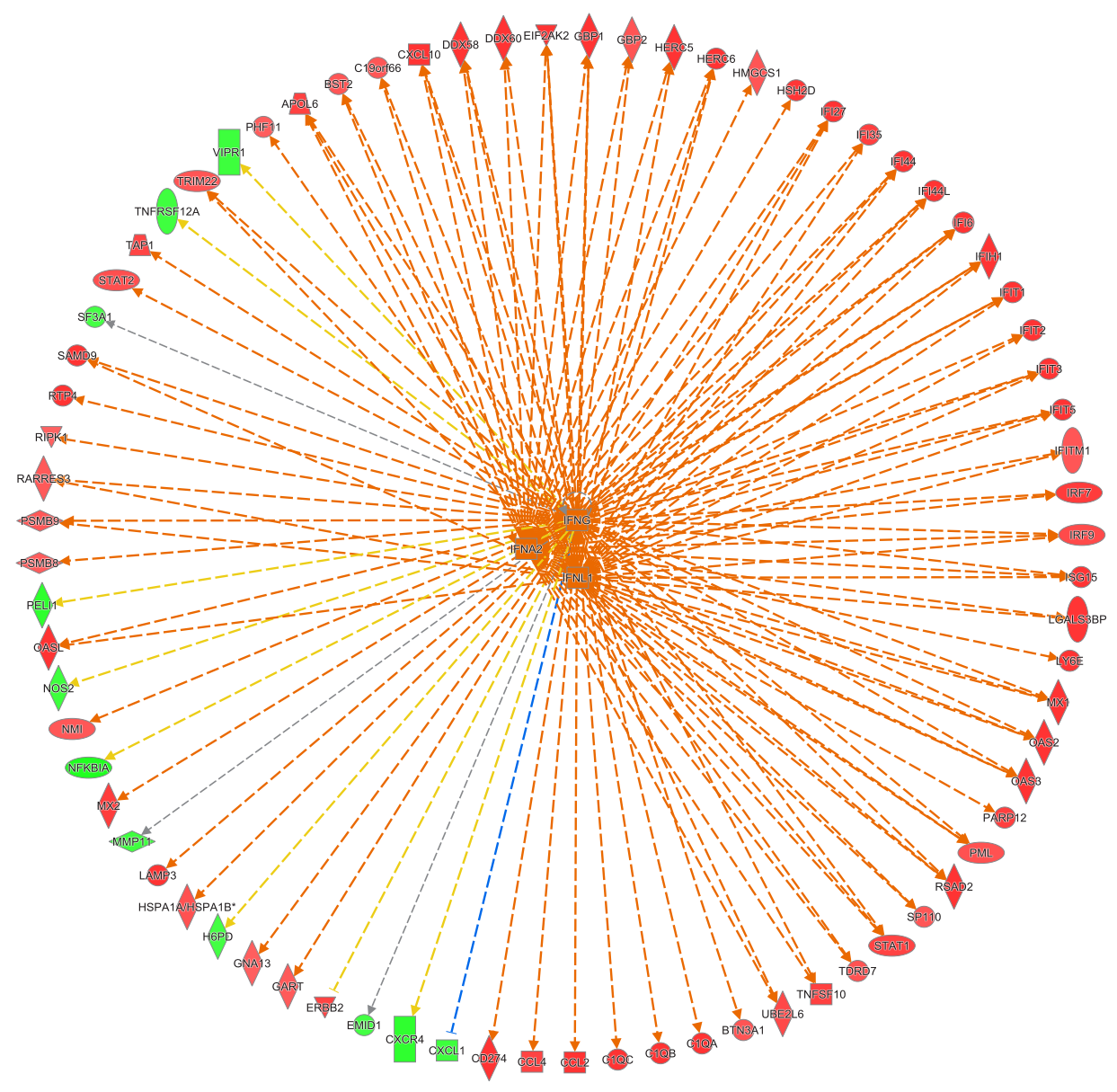

Figure 3-3. Genes of which the expression is known to be regulated by IFNA2, IFNG and IFNL1 that are significantly differentially expressed at baseline in APOE4 carriers compared to non-carriers.

Red indicates a significantly higher expression, green indicates a significantly lower expression $(P<0.05)$. Orange arrow, gene is predicted to be activated by regulator; blue arrow, gene is predicted to be inhibited by regulator; yellow arrow, gene expression of downstream gene is inconsistent with predicted state. 


\begin{tabular}{|c|c|c|c|c|}
\hline & & & APOE4 carriers & APOE4 non-carriers \\
\hline Ingenuity Canonical Pathways & $P$ value & Total genes & Up Down & Up Down \\
\hline Interferon Signaling & $6.26 \mathrm{E}-10$ & 34 & 27 & 93 \\
\hline $\begin{array}{l}\text { Activation of IRF by Cytosolic Pattern Recognition } \\
\text { Receptors }\end{array}$ & 7.67E-06 & 60 & 36 & 111 \\
\hline Protein Ubiquitination Pathway & $1.05 \mathrm{E}-04$ & 254 & 1014 & 2819 \\
\hline $\begin{array}{l}\text { Role of Pattern Recognition Receptors in } \\
\text { Recognition of Bacteria and Viruses }\end{array}$ & $1.79 E-04$ & 119 & 710 & 912 \\
\hline $\begin{array}{l}\text { Salvage Pathways of Pyrimidine } \\
\text { Deoxyribonucleotides }\end{array}$ & 2.07E-04 & 8 & 04 & 12 \\
\hline $\begin{array}{l}\text { Role of Osteoblasts, Osteoclasts and Chondrocytes } \\
\text { in Rheumatoid Arthritis }\end{array}$ & $9.58 \mathrm{E}-04$ & 214 & 104 & 814 \\
\hline UVA-Induced MAPK Signaling & $1.23 \mathrm{E}-03$ & 87 & 44 & 68 \\
\hline Death Receptor Signaling & $1.78 \mathrm{E}-03$ & 91 & 68 & 127 \\
\hline Epoxysqualene Biosynthesis & $1.85 \mathrm{E}-03$ & 2 & 01 & 01 \\
\hline Aldosterone Signaling in Epithelial Cells & $2.20 \mathrm{E}-03$ & 151 & 65 & 1219 \\
\hline
\end{tabular}

Figure 3-4. Top 10 pathways differentially regulated by fish-oil supplementation in APOE4 carriers compared to non-carriers.

The 10 canonical pathways with the lowest P-value are shown. The number of significantly up-regulated and down-regulated genes by fish-oil supplementation in APOE4 carriers and APOE4 non-carriers are shown. Total genes: total number of genes in the pathway, up (red): number of genes with significantly higher expression after intervention compared to before, down (green): number of genes with significantly lower expression after intervention compared to before. 


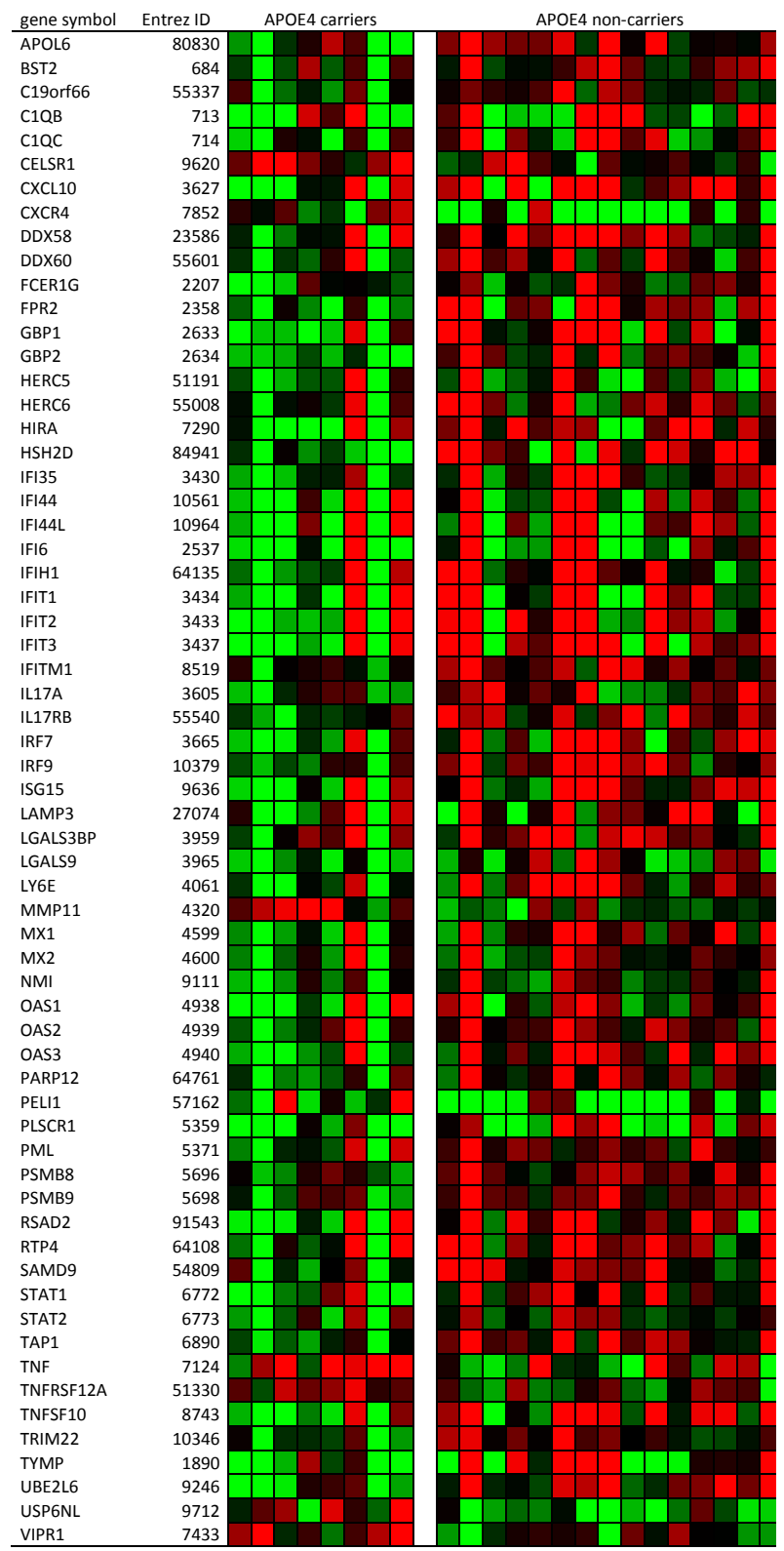

Log-ratio of expression after and before supplementation

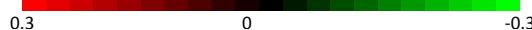

Figure 3-5. Heatmap depicting individual gene expression changes by fish-oil supplementation of genes that are significantly differently changed between carriers and non-carriers of APOE4 in response to fishoil supplementation and are known to be regulated by IFNL1, IFNA2 and IFNG.

Log-ratios of the expression after and before supplementation are shown. 


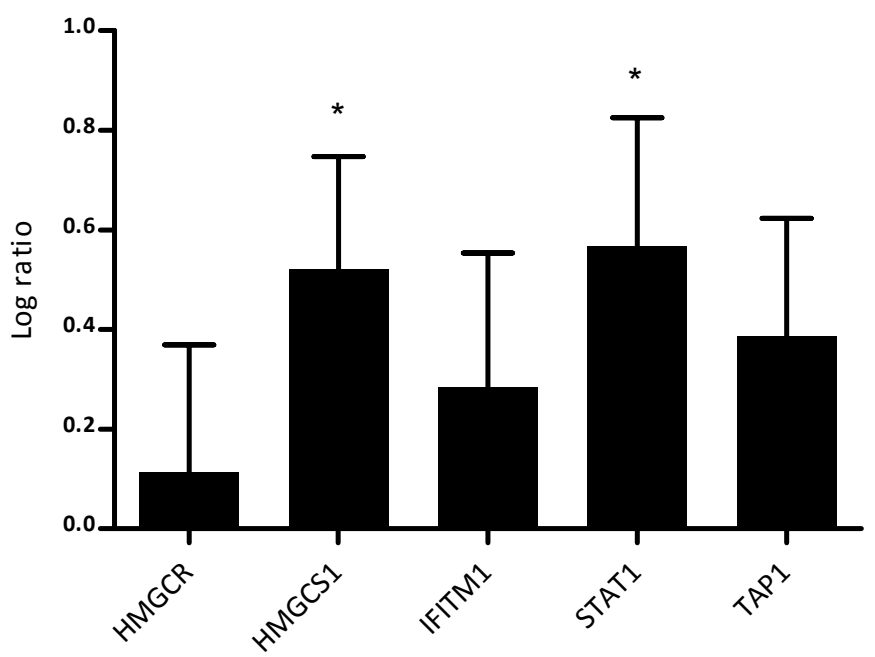

Figure 3-6. QPCR results of a selection of genes showing the log ratio of expression in APOE4 carriers compared to non-carriers.

Values are mean \pm SEM. * $p<0.05$ in carriers compared to non-carriers. HMGCR, 3-hydroxy-3-methylglutaryl-CoA reductase; HMGCS1, 3-hydroxy-3-methylglutaryl-CoA synthase 1; IFITM1, IFN induced transmembrane protein 1; STAT1, signal transducer and activator of transcription 1; TAP1, transporter 1, ATP-binding cassette, sub-family B. 


\section{Supplemental Tables}

Supplementary Table S3-1. CEL-file names, APOE genoype and subject IDs used in microarray analysis

\begin{tabular}{|c|c|c|}
\hline Filename & APOE genotype & Subject ID \\
\hline A101_003_8030_EPADHA_base.CEL & APOE4 carrier & 8030 \\
\hline A101_004_8169_EPADHA_base.CEL & APOE4 non-carrier & 8169 \\
\hline A101_005_8253_EPADHA_base.CEL & APOE4 non-carrier & 8253 \\
\hline A101_006_8263_EPADHA_base.CEL & APOE4 non-carrier & 8263 \\
\hline A101_007_8282_EPADHA_base.CEL & APOE4 non-carrier & 8282 \\
\hline A101_008_8299_EPADHA_base.CEL & APOE4 non-carrier & 8299 \\
\hline A101_009_8300_EPADHA_base.CEL & APOE4 non-carrier & 8300 \\
\hline A101_010_8337_EPADHA_base.CEL & APOE4 carrier & 8337 \\
\hline A101_011A_8345_EPADHA_base.CEL & APOE4 carrier & 8345 \\
\hline A101_012A_8346_EPADHA_base.CEL & APOE4 non-carrier & 8346 \\
\hline A101_013_8363_EPADHA_base.CEL & APOE4 carrier & 8363 \\
\hline A101_014_8364_EPADHA_base.CEL & APOE4 carrier & 8364 \\
\hline A101_015_8037_EPADHA_base.CEL & APOE4 non-carrier & 8037 \\
\hline A101_016_8145_EPADHA_base.CEL & APOE4 non-carrier & 8145 \\
\hline A101_017_8186_EPADHA_base.CEL & APOE4 carrier & 8186 \\
\hline A101_018_8200_EPADHA_base.CEL & APOE4 non-carrier & 8200 \\
\hline A101_019A_8233_EPADHA_base.CEL & APOE4 carrier & 8233 \\
\hline A101_020_8289_EPADHA_base.CEL & APOE4 non-carrier & 8289 \\
\hline A101_021_8294_EPADHA_base.CEL & APOE4 non-carrier & 8294 \\
\hline A101_022_8303_EPADHA_base.CEL & APOE4 non-carrier & 8303 \\
\hline A101_023_8305_EPADHA_base.CEL & APOE4 non-carrier & 8305 \\
\hline A101_024_8320_EPADHA_base.CEL & APOE4 non-carrier & 8320 \\
\hline A101_025_8331_EPADHA_base.CEL & APOE4 carrier & 8331 \\
\hline A101_054_8030_EPADHA_end.CEL & APOE4 carrier & 8030 \\
\hline A101_055_8169_EPADHA_end.CEL & APOE4 non-carrier & 8169 \\
\hline A101_056_8253_EPADHA_end.CEL & APOE4 non-carrier & 8253 \\
\hline A101_057_8263_EPADHA_end.CEL & APOE4 non-carrier & 8263 \\
\hline A101_058_8282_EPADHA_end.CEL & APOE4 non-carrier & 8282 \\
\hline A101_059_8299_EPADHA_end.CEL & APOE4 non-carrier & 8299 \\
\hline A101_060_8300_EPADHA_end.CEL & APOE4 non-carrier & 8300 \\
\hline A101_061A_8337_EPADHA_end.CEL & APOE4 carrier & 8337 \\
\hline A101_062_8345_EPADHA_end.CEL & APOE4 carrier & 8345 \\
\hline A101_063_8346_EPADHA_end.CEL & APOE4 non-carrier & 8346 \\
\hline A101_064_8363_EPADHA_end.CEL & APOE4 carrier & 8363 \\
\hline A101_065_8364_EPADHA_end.CEL & APOE4 carrier & 8364 \\
\hline A101_066_8037_EPADHA_end.CEL & APOE4 non-carrier & 8037 \\
\hline A101_067_8145_EPADHA_end.CEL & APOE4 non-carrier & 8145 \\
\hline A101_068_8186_EPADHA_end.CEL & APOE4 carrier & 8186 \\
\hline A101_069_8200_EPADHA_end.CEL & APOE4 non-carrier & 8200 \\
\hline A101_070_8233_EPADHA_end.CEL & APOE4 carrier & 8233 \\
\hline A101_071_8289_EPADHA_end.CEL & APOE4 non-carrier & 8289 \\
\hline A101_072_8294_EPADHA_end.CEL & APOE4 non-carrier & 8294 \\
\hline A101_073_8303_EPADHA_end.CEL & APOE4 non-carrier & 8303 \\
\hline A101_074_8305_EPADHA_end.CEL & APOE4 non-carrier & 8305 \\
\hline
\end{tabular}




\begin{tabular}{lll}
\hline Filename & APOE genotype & Subject ID \\
\hline A101_075_8320_EPADHA_end.CEL & APOE4 non-carrier & 8320 \\
A101_076_8331_EPADHA_end.CEL & APOE4 carrier & 8331 \\
\hline
\end{tabular}

Supplementary Table S3-2. Overrepresented gene sets at baseline when comparing APOE4 carriers to non-carriers using GSEA.

\begin{tabular}{|c|c|}
\hline NAME & FDR Q-value \\
\hline INTERFERON.ALPHA.BETA.SIGNALING & 0.00 \\
\hline WP1835.INTERFERON.ALPHA.BETA.SIGNALING & 0.00 \\
\hline INTERFERON.SIGNALING & 0.00 \\
\hline GENERIC.TRANSCRIPTION.PATHWAY & 0.00 \\
\hline HSA05162 & 0.00 \\
\hline ANTIVIRAL.MECHANISM.BY.IFN.STIMULATED.GENES & 0.00 \\
\hline S.PHASE & 0.00 \\
\hline ISG15.ANTIVIRAL.MECHANISM & 0.00 \\
\hline SYNTHESIS.OF.DNA & 0.00 \\
\hline DNA.REPLICATION & 0.00 \\
\hline HSA05168 & 0.00 \\
\hline WP384.APOPTOSIS.MODULATION.BY.HSP70 & 0.00 \\
\hline METABOLISM.OF.NON.CODING.RNA & 0.00 \\
\hline CYTOKINE.SIGNALING.IN.IMMUNE.SYSTEM & 0.00 \\
\hline ANTIGEN.PROCESSING.UBIQUITINATION.PROTEASOME.DEGRADATION & 0.00 \\
\hline WP2715.METABOLISM.OF.NON.CODING.RNA & 0.00 \\
\hline WP1925.SYNTHESIS.OF.DNA & 0.00 \\
\hline SNRNP.ASSEMBLY & 0.00 \\
\hline DNA.STRAND.ELONGATION & 0.00 \\
\hline ACTIVATION.OF.ATR.IN.RESPONSE.TO.REPLICATION.STRESS & 0.00 \\
\hline INTERFERON.GAMMA.SIGNALING & 0.00 \\
\hline CELL.CYCLE & 0.00 \\
\hline WP2757.MITOTIC.METAPHASE.AND.ANAPHASE & 0.00 \\
\hline WP2772.S.PHASE & 0.00 \\
\hline TRAF6.MEDIATED.IRF7.ACTIVATION & 0.00 \\
\hline MITOTIC.METAPHASE.AND.ANAPHASE & 0.01 \\
\hline CELL.CYCLE.MITOTIC & 0.00 \\
\hline CLASS.I.MHC.MEDIATED.ANTIGEN.PROCESSING.PRESENTATION & 0.01 \\
\hline WP2785.M.G1.TRANSITION & 0.01 \\
\hline MITOTIC.ANAPHASE & 0.01 \\
\hline WP1775.CELL.CYCLE.CHECKPOINTS & 0.01 \\
\hline HSA05164 & 0.01 \\
\hline HSA03460 & 0.01 \\
\hline HSA04622 & 0.01 \\
\hline WP2752.MYD88.INDEPENDENT.CASCADE. & 0.01 \\
\hline HSA05160 & 0.01 \\
\hline RIG.I.MDA5.MEDIATED.INDUCTION.OF.IFN.ALPHA.BETA.PATHWAYS & 0.01 \\
\hline HSA04623 & 0.01 \\
\hline WP197.CHOLESTEROL.BIOSYNTHESIS & 0.01 \\
\hline G1.S.TRANSITION & 0.01 \\
\hline CHOLESTEROL.BIOSYNTHESIS & 0.01 \\
\hline
\end{tabular}




\begin{tabular}{|c|c|}
\hline NAME & FDR Q-value \\
\hline SEPARATION.OF.SISTER.CHROMATIDS & 0.01 \\
\hline WP1904.RIG.I.MDA5.MEDIATED.INDUCTION.OF.IFN.ALPHA.BETA.PATHWAYS & 0.01 \\
\hline MITOTIC.M.M.G1.PHASES & 0.01 \\
\hline PROCESSING.OF.CAPPED.INTRONLESS.PRE.MRNA & 0.01 \\
\hline REMOVAL.OF.LICENSING.FACTORS.FROM.ORIGINS & 0.01 \\
\hline POST.ELONGATION.PROCESSING.OF.INTRONLESS.PRE.MRNA & 0.01 \\
\hline CYCLIN.A.CDK2.ASSOCIATED.EVENTS.AT.S.PHASE.ENTRY & 0.01 \\
\hline HSA03008 & 0.01 \\
\hline POST.ELONGATION.PROCESSING.OF.THE.TRANSCRIPT & 0.01 \\
\hline HSA03050 & 0.01 \\
\hline EXPORT.OF.VIRAL.RIBONUCLEOPROTEINS.FROM.NUCLEUS & 0.01 \\
\hline PPARA.ACTIVATES.GENE.EXPRESSION & 0.01 \\
\hline M.G1.TRANSITION & 0.01 \\
\hline WP1898.REGULATION.OF.DNA.REPLICATION & 0.01 \\
\hline METABOLISM.OF.RNA & 0.01 \\
\hline WP2733.REGULATION.OF.MRNA.STABILITY.BY.PROTEINS.THAT.BIND.AU.RICH.ELEMENTS & 0.01 \\
\hline REGULATION.OF.MITOTIC.CELL.CYCLE & 0.01 \\
\hline APC.C.MEDIATED.DEGRADATION.OF.CELL.CYCLE.PROTEINS & 0.01 \\
\hline REGULATION.OF.GLUCOKINASE.BY.GLUCOKINASE.REGULATORY.PROTEIN & 0.01 \\
\hline MITOTIC.G1.G1.S.PHASES & 0.01 \\
\hline G2.M.CHECKPOINTS & 0.01 \\
\hline CELL.CYCLE.CHECKPOINTS & 0.01 \\
\hline WP619.TYPE.II.INTERFERON.SIGNALING.IFNG. & 0.01 \\
\hline WP183.PROTEASOME.DEGRADATION & 0.01 \\
\hline LAGGING.STRAND.SYNTHESIS & 0.01 \\
\hline & 0.01 \\
\hline TELOMERE.C.STRAND.LAGGING.STRAND.SYNTHESIS & 0.01 \\
\hline DNA.REPLICATION.PRE.INITIATION & 0.01 \\
\hline TRANSPORT.OF.MATURE.TRANSCRIPT.TO.CYTOPLASM & 0.01 \\
\hline REGULATION.OF.APC.C.ACTIVATORS.BETWEEN.G1.S.AND.EARLY.ANAPHASE & 0.01 \\
\hline RNA.POLYMERASE.II.TRANSCRIPTION.TERMINATION & 0.01 \\
\hline SCF.BETA.TRCP.MEDIATED.DEGRADATION.OF.EMI1 & 0.01 \\
\hline AUF1.HNRNP.D0.DESTABILIZES.MRNA & 0.01 \\
\hline BIOC_CASPASEPATHWAY & 0.01 \\
\hline HSA04612 & 0.01 \\
\hline NEGATIVE.REGULATORS.OF.RIG.I.MDA5.SIGNALING & 0.01 \\
\hline SWITCHING.OF.ORIGINS.TO.A.POST.REPLICATIVE.STATE & 0.01 \\
\hline REV.MEDIATED.NUCLEAR.EXPORT.OF.HIV.RNA & 0.01 \\
\hline CYCLIN.E.ASSOCIATED.EVENTS.DURING.G1.S.TRANSITION & 0.01 \\
\hline WP1782.APC.C.MEDIATED.DEGRADATION.OF.CELL.CYCLE.PROTEINS & 0.01 \\
\hline TRANSPORT.OF.THE.SLBP.DEPENDANT.MATURE.MRNA & 0.01 \\
\hline M.PHASE & 0.01 \\
\hline ORC1.REMOVAL.FROM.CHROMATIN & 0.01 \\
\hline HSA04940 & 0.01 \\
\hline NEP.NS2.INTERACTS.WITH.THE.CELLULAR.EXPORT.MACHINERY & 0.01 \\
\hline WP2672.ISG15.ANTIVIRAL.MECHANISM & 0.01 \\
\hline TRANSPORT.OF.MATURE.MRNAS.DERIVED.FROM.INTRONLESS.TRANSCRIPTS & 0.01 \\
\hline EXTENSION.OF.TELOMERES & 0.01 \\
\hline
\end{tabular}


APOE4 and fish-oil

\begin{tabular}{|c|c|}
\hline NAME & FDR Q-value \\
\hline HSA03030 & 0.01 \\
\hline APC.C.CDC20.MEDIATED.DEGRADATION.OF.SECURIN & 0.01 \\
\hline ER.PHAGOSOME.PATHWAY & 0.01 \\
\hline WP1795.CHOLESTEROL.BIOSYNTHESIS & 0.01 \\
\hline CLEAVAGE.OF.GROWING.TRANSCRIPT.IN.THE.TERMINATION.REGION & 0.01 \\
\hline REGULATION.OF.CHOLESTEROL.BIOSYNTHESIS.BY.SREBP.SREBF. & 0.01 \\
\hline WP75.TOLL.LIKE.RECEPTOR.SIGNALING.PATHWAY & 0.01 \\
\hline BIOC_ATRBRCAPATHWAY & 0.01 \\
\hline TRANSPORT.OF.THE.SLBP.INDEPENDENT.MATURE.MRNA & 0.01 \\
\hline WP2796.CLASS.I.MHC.MEDIATED.ANTIGEN.PROCESSING.AMP.PRESENTATION & 0.01 \\
\hline $\begin{array}{l}\text { WP2797.REGULATION.OF.LIPID.METABOLISM.BY.PEROXISOME.PROLIFERATOR.ACTIVATED. } \\
\text { RECEPTOR.ALPHA.PPARALPHA. }\end{array}$ & 0.01 \\
\hline AUTODEGRADATION.OF.CDH1.BY.CDH1.APC.C & 0.01 \\
\hline REGULATION.OF.DNA.REPLICATION & 0.01 \\
\hline VIF.MEDIATED.DEGRADATION.OF.APOBEC3G & 0.01 \\
\hline SCF.SKP2.MEDIATED.DEGRADATION.OF.P27.P21 & 0.01 \\
\hline $\begin{array}{l}\text { REGULATION.OF.LIPID.METABOLISM.BY.PEROXISOME.PROLIFERATOR.ACTIVATED.RECEPTOR. } \\
\text { ALPHA.PPARALPHA. }\end{array}$ & 0.01 \\
\hline REGULATION.OF.MRNA.STABILITY.BY.PROTEINS.THAT.BIND.AU.RICH.ELEMENTS & 0.01 \\
\hline TRANSPORT.OF.MATURE.MRNA.DERIVED.FROM.AN.INTRONLESS.TRANSCRIPT & 0.01 \\
\hline HSA00900 & 0.01 \\
\hline HSA04120 & 0.01 \\
\hline IKK.COMPLEX.RECRUITMENT.MEDIATED.BY.RIP1 & 0.01 \\
\hline WP1858.MITOTIC.G1.G1.S.PHASES & 0.01 \\
\hline TRANSPORT.OF.MATURE.MRNA.DERIVED.FROM.AN.INTRON.CONTAINING.TRANSCRIPT & 0.01 \\
\hline TRANSPORT.OF.RIBONUCLEOPROTEINS.INTO.THE.HOST.NUCLEUS & 0.01 \\
\hline BIOC_PROTEASOMEPATHWAY & 0.01 \\
\hline HSA05330 & 0.01 \\
\hline WP466.DNA.REPLICATION & 0.02 \\
\hline HSA00970 & 0.02 \\
\hline HSA04620 & 0.02 \\
\hline CDK.MEDIATED.PHOSPHORYLATION.AND.REMOVAL.OF.CDC6 & 0.02 \\
\hline CDC20.PHOSPHO.APC.C.MEDIATED.DEGRADATION.OF.CYCLIN.A & 0.02 \\
\hline CDT1.ASSOCIATION.WITH.THE.CDC6.ORC.ORIGIN.COMPLEX & 0.02 \\
\hline APC.C.CDC20.MEDIATED.DEGRADATION.OF.MITOTIC.PROTEINS & 0.02 \\
\hline GLI3.IS.PROCESSED.TO.GLI3R.BY.THE.PROTEASOME & 0.02 \\
\hline WP2659.DEADENYLATION.DEPENDENT.MRNA.DECAY & 0.02 \\
\hline DEGRADATION.OF.AXIN & 0.02 \\
\hline WP2706.ACTIVATION.OF.GENE.EXPRESSION.BY.SREBP.SREBF. & 0.02 \\
\hline WP314.FAS.PATHWAY.AND.STRESS.INDUCTION.OF.HSP.REGULATION & 0.02 \\
\hline RNA.POLYMERASE.II.TRANSCRIPTION & 0.02 \\
\hline WP254.APOPTOSIS & 0.02 \\
\hline WP2773.DEGRADATION.OF.BETA.CATENIN.BY.THE.DESTRUCTION.COMPLEX & 0.02 \\
\hline NUCLEAR.IMPORT.OF.REV.PROTEIN & 0.02 \\
\hline ACTIVATION.OF.GENE.EXPRESSION.BY.SREBF.SREBP. & 0.02 \\
\hline RNA.POLYMERASE.I.PROMOTER.ESCAPE & 0.02 \\
\hline RESOLUTION.OF.SISTER.CHROMATID.COHESION & 0.02 \\
\hline P53.DEPENDENT.G1.S.DNA.DAMAGE.CHECKPOINT & 0.02 \\
\hline AUTODEGRADATION.OF.THE.E3.UBIQUITIN.LIGASE.COP1 & 0.02 \\
\hline ASSEMBLY.OF.THE.PRE.REPLICATIVE.COMPLEX & 0.02 \\
\hline
\end{tabular}




\begin{tabular}{|c|c|}
\hline NAME & FDR Q-value \\
\hline ACTIVATION.OF.THE.PRE.REPLICATIVE.COMPLEX & 0.02 \\
\hline G1.S.DNA.DAMAGE.CHECKPOINTS & 0.02 \\
\hline INTERACTIONS.OF.REV.WITH.HOST.CELLULAR.PROTEINS & 0.02 \\
\hline WP2652.MITOTIC.PROMETAPHASE & 0.02 \\
\hline WP1896.REGULATION.OF.APOPTOSIS & 0.02 \\
\hline CROSS.PRESENTATION.OF.SOLUBLE.EXOGENOUS.ANTIGENS.ENDOSOMES. & 0.02 \\
\hline MITOCHONDRIAL.TRANSLATION.INITIATION & 0.02 \\
\hline BIOC_DEATHPATHWAY & 0.02 \\
\hline DEGRADATION.OF.GLI1.BY.THE.PROTEASOME & 0.02 \\
\hline P53.INDEPENDENT.DNA.DAMAGE.RESPONSE & 0.02 \\
\hline P53.DEPENDENT.G1.DNA.DAMAGE.RESPONSE & 0.02 \\
\hline DEADENYLATION.DEPENDENT.MRNA.DECAY & 0.02 \\
\hline POST.ELONGATION.PROCESSING.OF.INTRON.CONTAINING.PRE.MRNA & 0.02 \\
\hline DEGRADATION.OF.DVL & 0.02 \\
\hline HSA05332 & 0.02 \\
\hline MITOTIC.PROMETAPHASE & 0.02 \\
\hline S37.MUTANTS.OF.BETA.CATENIN.AREN.T.PHOSPHORYLATED & 0.02 \\
\hline $\begin{array}{l}\text { PHOSPHORYLATION.SITE.MUTANTS.OF.CTNNB1.ARE.NOT.TARGETED.TO.THE.PROTEASOME. } \\
\text { BY.THE.DESTRUCTION.COMPLEX }\end{array}$ & 0.02 \\
\hline METABOLISM.OF.MRNA & 0.02 \\
\hline P53.INDEPENDENT.G1.S.DNA.DAMAGE.CHECKPOINT & 0.02 \\
\hline STABILIZATION.OF.P53 & 0.02 \\
\hline AMER1.MUTANTS.DESTABILIZE.THE.DESTRUCTION.COMPLEX & 0.02 \\
\hline REGULATION.OF.ACTIVATED.PAK.2P34.BY.PROTEASOME.MEDIATED.DEGRADATION & 0.02 \\
\hline WP1890.PROCESSING.OF.CAPPED.INTRONLESS.PRE.MRNA & 0.02 \\
\hline DELETIONS.IN.THE.AMER1.GENE.DESTABILIZE.THE.DESTRUCTION.COMPLEX & 0.03 \\
\hline UBIQUITIN.DEPENDENT.DEGRADATION.OF.CYCLIN.D1 & 0.03 \\
\hline WP1807.DOUBLE.STRAND.BREAK.REPAIR & 0.03 \\
\hline MRNA.3.END.PROCESSING & 0.03 \\
\hline ACTIVATION.OF.APC.C.AND.APC.C.CDC20.MEDIATED.DEGRADATION.OF.MITOTIC.PROTEINS & 0.03 \\
\hline ACTIVATION.OF.NF.KAPPAB.IN.B.CELLS & 0.03 \\
\hline UBIQUITIN.MEDIATED.DEGRADATION.OF.PHOSPHORYLATED.CDC25A & 0.03 \\
\hline CHROMOSOME.MAINTENANCE & 0.03 \\
\hline WP2507.NANOMATERIAL.INDUCED.APOPTOSIS & 0.03 \\
\hline T41.MUTANTS.OF.BETA.CATENIN.AREN.T.PHOSPHORYLATED & 0.03 \\
\hline S33.MUTANTS.OF.BETA.CATENIN.AREN.T.PHOSPHORYLATED & 0.03 \\
\hline AXIN.MISSENSE.MUTANTS.DESTABILIZE.THE.DESTRUCTION.COMPLEX & 0.03 \\
\hline $\begin{array}{l}\text { DELETIONS.IN.THE.AXIN.GENES.IN.HEPATOCELLULAR.CARCINOMA.RESULT.IN.ELEVATED.WNT. } \\
\text { SIGNALING }\end{array}$ & 0.03 \\
\hline DEGRADATION.OF.GLI2.BY.THE.PROTEASOME & 0.03 \\
\hline MISSPLICED.GSK3BETA.MUTANTS.STABILIZE.BETA.CATENIN & 0.03 \\
\hline TRUNCATIONS.OF.AMER1.DESTABILIZE.THE.DESTRUCTION.COMPLEX & 0.03 \\
\hline DEGRADATION.OF.BETA.CATENIN.BY.THE.DESTRUCTION.COMPLEX & 0.03 \\
\hline RNA.POLYMERASE.I.TRANSCRIPTION.INITIATION & 0.03 \\
\hline VPR.MEDIATED.NUCLEAR.IMPORT.OF.PICS & 0.03 \\
\hline HSA05161 & 0.03 \\
\hline MITOCHONDRIAL.TRANSLATION.TERMINATION & 0.03 \\
\hline S45.MUTANTS.OF.BETA.CATENIN.AREN.T.PHOSPHORYLATED & 0.03 \\
\hline HSA03022 & 0.03 \\
\hline TRUNCATED.APC.MUTANTS.DESTABILIZE.THE.DESTRUCTION.COMPLEX & 0.03 \\
\hline
\end{tabular}


APOE4 and fish-oil

\begin{tabular}{|c|c|}
\hline NAME & FDR Q-value \\
\hline HH.LIGAND.BIOGENESIS.DISEASE & 0.03 \\
\hline AXIN.MUTANTS.DESTABILIZE.THE.DESTRUCTION.COMPLEX.ACTIVATING.WNT.SIGNALING & 0.03 \\
\hline TCF7L2.MUTANTS.DON.T.BIND.CTBP & 0.03 \\
\hline NUCLEAR.PORE.COMPLEX.NPC.DISASSEMBLY & 0.03 \\
\hline WP1906.RNA.POLYMERASE.II.TRANSCRIPTION & 0.03 \\
\hline UBIQUITIN.DEPENDENT.DEGRADATION.OF.CYCLIN.D & 0.03 \\
\hline APC.TRUNCATION.MUTANTS.ARE.NOT.K63.POLYUBIQUITINATED & 0.03 \\
\hline WP1816.FANCONI.ANEMIA.PATHWAY & 0.03 \\
\hline BIOC_IL12PATHWAY & 0.03 \\
\hline FANCONI.ANEMIA.PATHWAY & 0.03 \\
\hline APC.TRUNCATION.MUTANTS.HAVE.IMPAIRED.AXIN.BINDING & 0.03 \\
\hline HSA03440 & 0.03 \\
\hline PROCESSIVE.SYNTHESIS.ON.THE.LAGGING.STRAND & 0.03 \\
\hline MITOCHONDRIAL.TRANSLATION.ELONGATION & 0.04 \\
\hline PROCESSING.DEFECTIVE.HH.VARIANTS.ABROGATE.LIGAND.SECRETION & 0.04 \\
\hline HIV.LIFE.CYCLE & 0.04 \\
\hline DOUBLE.STRAND.BREAK.REPAIR & 0.04 \\
\hline CONVERSION.FROM.APC.C.CDC20.TO.APC.C.CDH1.IN.LATE.ANAPHASE & 0.04 \\
\hline MITOCHONDRIAL.TRANSLATION & 0.04 \\
\hline TRNA.AMINOACYLATION & 0.04 \\
\hline REGULATION.OF.HSF1.MEDIATED.HEAT.SHOCK.RESPONSE & 0.04 \\
\hline HSA03013 & 0.04 \\
\hline LATE.PHASE.OF.HIV.LIFE.CYCLE & 0.05 \\
\hline ORGANELLE.BIOGENESIS.AND.MAINTENANCE & 0.05 \\
\hline HEDGEHOG.LIGAND.BIOGENESIS & 0.05 \\
\hline WP391.MITOCHONDRIAL.GENE.EXPRESSION & 0.05 \\
\hline WP2751.TRANSCRIPTIONAL.REGULATION.OF.WHITE.ADIPOCYTE.DIFFERENTIATION & 0.05 \\
\hline REGULATION.OF.PLK1.ACTIVITY.AT.G2.M.TRANSITION & 0.05 \\
\hline WP2794.CYTOSOLIC.SENSORS.OF.PATHOGEN.ASSOCIATED.DNA. & 0.05 \\
\hline WP2328.ALLOGRAFT.REJECTION & 0.05 \\
\hline G2.M.TRANSITION & 0.05 \\
\hline WP2658.HIV.LIFE.CYCLE & 0.05 \\
\hline WP1982.SREBP.SIGNALLING & 0.05 \\
\hline VPU.MEDIATED.DEGRADATION.OF.CD4 & 0.05 \\
\hline WP45.G1.TO.S.CELL.CYCLE.CONTROL & 0.05 \\
\hline RNA.POLYMERASE.II.PROMOTER.ESCAPE & 0.05 \\
\hline HSA04710 & 0.05 \\
\hline GAP.FILLING.DNA.REPAIR.SYNTHESIS.AND.LIGATION.IN.TC.NER & 0.05 \\
\hline ANTIGEN.PRESENTATION.FOLDING.ASSEMBLY.AND.PEPTIDE.LOADING.OF.CLASS.I.MHC & 0.05 \\
\hline REGULATION.OF.APOPTOSIS & 0.05 \\
\hline NOD1.2.SIGNALING.PATHWAY & 0.05 \\
\hline GLOBAL.GENOMIC.NER.GG.NER. & 0.05 \\
\hline ACTIVATION.OF.IRF3.IRF7.MEDIATED.BY.TBK1.IKK.EPSILON & 0.05 \\
\hline HIV.INFECTION & 0.05 \\
\hline RNA.POLYMERASE.II.TRANSCRIPTION.PRE.INITIATION.AND.PROMOTER.OPENING & 0.05 \\
\hline BIOC_MITOCHONDRIAPATHWAY & 0.05 \\
\hline HSA04668 & 0.05 \\
\hline TELOMERE.MAINTENANCE & 0.05 \\
\hline
\end{tabular}




\begin{tabular}{|c|c|}
\hline NAME & FDR Q-value \\
\hline BIOC_NKTPATHWAY & 0.05 \\
\hline TRANSCRIPTIONAL.REGULATION.OF.WHITE.ADIPOCYTE.DIFFERENTIATION & 0.06 \\
\hline RNA.POLYMERASE.II.HIV.PROMOTER.ESCAPE & 0.06 \\
\hline RNA.POLYMERASE.II.TRANSCRIPTION.INITIATION.AND.PROMOTER.CLEARANCE & 0.06 \\
\hline WP1859.MITOTIC.G2.G2.M.PHASES & 0.06 \\
\hline RNA.POLYMERASE.II.TRANSCRIPTION.INITIATION & 0.06 \\
\hline GAP.FILLING.DNA.REPAIR.SYNTHESIS.AND.LIGATION.IN.GG.NER & 0.06 \\
\hline HIV.TRANSCRIPTION.INITIATION & 0.06 \\
\hline HSA03018 & 0.06 \\
\hline REGULATION.OF.ORNITHINE.DECARBOXYLASE.ODC. & 0.06 \\
\hline RNA.POLYMERASE.ITRANSCRIPTION.TERMINATION & 0.06 \\
\hline WP2363.GASTRIC.CANCER.NETWORK.2 & 0.06 \\
\hline WP2446.RB.IN.CANCER & 0.06 \\
\hline CHEMOKINE.RECEPTORS.BIND.CHEMOKINES & 0.07 \\
\hline NUCLEAR.ENVELOPE.BREAKDOWN & 0.07 \\
\hline ANTIGEN.PROCESSING.CROSS.PRESENTATION & 0.07 \\
\hline DNA.REPAIR & 0.07 \\
\hline APOPTOSIS & 0.07 \\
\hline ASYMMETRIC.LOCALIZATION.OF.PCP.PROTEINS & 0.07 \\
\hline BUTYRATE.RESPONSE.FACTOR.1.BRF1.DESTABILIZES.MRNA & 0.07 \\
\hline PROCESSING.OF.CAPPED.INTRON.CONTAINING.PRE.MRNA & 0.07 \\
\hline HSA05142 & 0.07 \\
\hline TRANSCRIPTION.OF.THE.HIV.GENOME & 0.07 \\
\hline WP1822.GENERIC.TRANSCRIPTION.PATHWAY & 0.07 \\
\hline WP2746.SIGNALING.BY.THE.B.CELL.RECEPTOR.BCR. & 0.07 \\
\hline WP2717.MITOCHONDRIAL.PROTEIN.IMPORT & 0.07 \\
\hline VIRAL.MESSENGER.RNA.SYNTHESIS & 0.07 \\
\hline MITOTIC.G2.G2.M.PHASES & 0.08 \\
\hline WP1928.TELOMERE.MAINTENANCE & 0.08 \\
\hline WP2654.MITOTIC.PROPHASE & 0.08 \\
\hline FATTY.ACID.TRIACYLGLYCEROL.AND.KETONE.BODY.METABOLISM & 0.08 \\
\hline WP405.EUKARYOTIC.TRANSCRIPTION.INITIATION & 0.08 \\
\hline BIOC_VEGFPATHWAY & 0.08 \\
\hline TRANSCRIPTION & 0.08 \\
\hline WP1449.REGULATION.OF.TOLL.LIKE.RECEPTOR.SIGNALING.PATHWAY & 0.08 \\
\hline WP1785.ASPARAGINE.N.LINKED.GLYCOSYLATION & 0.09 \\
\hline HSA05321 & 0.09 \\
\hline CYTOSOLIC.SENSORS.OF.PATHOGEN.ASSOCIATED.DNA & 0.09 \\
\hline PERK.REGULATES.GENE.EXPRESSION & 0.10 \\
\hline WP1938.TRNA.AMINOACYLATION & 0.10 \\
\hline
\end{tabular}

Supplementary Table S3-3. Underrepresented gene sets at baseline when comparing APOE4 carriers to non-carriers using GSEA.

\begin{tabular}{ll}
\hline NAME & FDR Q-value \\
\hline OLFACTORY.SIGNALING.PATHWAY & 0.00 \\
HSA04740 & 0.00 \\
VOLTAGE.GATED.POTASSIUM.CHANNELS & 0.01
\end{tabular}




\begin{tabular}{|c|c|}
\hline NAME & FDR Q-value \\
\hline WP2669.POTASSIUM.CHANNELS & 0.01 \\
\hline HSA04080 & 0.01 \\
\hline POTASSIUM.CHANNELS & 0.01 \\
\hline ACTIVATION.OF.MATRIX.METALLOPROTEINASES & 0.01 \\
\hline STRIATED.MUSCLE.CONTRACTION & 0.01 \\
\hline WP383.STRIATED.MUSCLE.CONTRACTION & 0.02 \\
\hline WP129.MATRIX.METALLOPROTEINASES & 0.02 \\
\hline WP1602.NICOTINE.ACTIVITY.ON.DOPAMINERGIC.NEURONS & 0.03 \\
\hline HSA05034 & 0.03 \\
\hline AMINE.COMPOUND.SLC.TRANSPORTERS & 0.05 \\
\hline COLLAGEN.FORMATION & 0.06 \\
\hline PLATELET.DEGRANULATION & 0.06 \\
\hline NEURONAL.SYSTEM & 0.06 \\
\hline WP1911.SIGNALING.BY.FGFR & 0.07 \\
\hline WP1811.EUKARYOTIC.TRANSLATION.ELONGATION & 0.07 \\
\hline RESPONSE.TO.ELEVATED.PLATELET.CYTOSOLIC.CA2. & 0.07 \\
\hline MUSCLE.CONTRACTION & 0.09 \\
\hline ASSEMBLY.OF.COLLAGEN.FIBRILS.AND.OTHER.MULTIMERIC.STRUCTURES & 0.09 \\
\hline WP2001.MIR.TARGETED.GENES.IN.ADIPOCYTES.TARBASE & 0.09 \\
\hline SIGNALING.BY.GPCR & 0.09 \\
\hline WP1864.MUSCLE.CONTRACTION & 0.09 \\
\hline WP325.TRIACYLGLYCERIDE.SYNTHESIS & 0.09 \\
\hline WP2267.SYNAPTIC.VESICLE.PATHWAY & 0.09 \\
\hline CLASS.B.2.SECRETIN.FAMILY.RECEPTORS. & 0.09 \\
\hline STIMULI.SENSING.CHANNELS & 0.10 \\
\hline COLLAGEN.DEGRADATION & 0.10 \\
\hline WP2855.DOPMINERGIC.NEUROGENESIS & 0.10 \\
\hline AMYLOIDS & 0.10 \\
\hline GPCR.DOWNSTREAM.SIGNALING & 0.10 \\
\hline
\end{tabular}

Supplementary Table S3-4. Overrepresented gene sets when comparing the response to fish-oil supplementation in APOE4 carriers to non-carriers using GSEA

\begin{tabular}{ll}
\hline NAME & FDR Q-value \\
\hline WP1811.EUKARYOTIC.TRANSLATION.ELONGATION & 0.01 \\
FORMATION.OF.A.POOL.OF.FREE.4OS.SUBUNITS & 0.01 \\
PEPTIDE.CHAIN.ELONGATION & 0.01 \\
X3.UTR.MEDIATED.TRANSLATIONAL.REGULATION & 0.01 \\
WP477.CYTOPLASMIC.RIBOSOMAL.PROTEINS & 0.01 \\
WP1813.EUKARYOTIC.TRANSLATION.TERMINATION & 0.01 \\
EUKARYOTIC.TRANSLATION.ELONGATION & 0.01 \\
L13A.MEDIATED.TRANSLATIONAL.SILENCING.OF.CERULOPLASMIN.EXPRESSION & 0.01 \\
GTP.HYDROLYSIS.AND.JOINING.OF.THE.6OS.RIBOSOMAL.SUBUNIT & 0.01 \\
EUKARYOTIC.TRANSLATION.TERMINATION & 0.02 \\
VIRAL.MRNA.TRANSLATION & 0.02 \\
BIOC_RAC1PATHWAY & 0.02 \\
BIOC_CTLA4PATHWAY & 0.02 \\
CONSTITUTIVE.PI3K.AKT.SIGNALING.IN.CANCER & 0.02
\end{tabular}




\begin{tabular}{|c|c|}
\hline NAME & FDR Q-value \\
\hline NONSENSE.MEDIATED.DECAY.NMD.INDEPENDENT.OF.THE.EXON.JUNCTION.COMPLEX.EJC. & 0.02 \\
\hline WP2677.SIGNALING.BY.TYPE.1.INSULIN.LIKE.GROWTH.FACTOR.1.RECEPTOR.IGF1R. & 0.04 \\
\hline PIP3.ACTIVATES.AKT.SIGNALING & 0.04 \\
\hline WP1812.EUKARYOTIC.TRANSLATION.INITIATION & 0.04 \\
\hline PI3K.AKT.ACTIVATION & 0.05 \\
\hline PI3K.EVENTS.IN.ERBB4.SIGNALING & 0.05 \\
\hline NEPHRIN.INTERACTIONS & 0.05 \\
\hline PI.3K.CASCADE & 0.05 \\
\hline PI3K.EVENTS.IN.ERBB2.SIGNALING & 0.05 \\
\hline PI3K.AKT.SIGNALING.IN.CANCER & 0.05 \\
\hline WP2261.SIGNALING.PATHWAYS.IN.GLIOBLASTOMA & 0.06 \\
\hline BIOC_EIF4PATHWAY & 0.07 \\
\hline EFFECTS.OF.PIP2.HYDROLYSIS & 0.07 \\
\hline WP1867.NEPHRIN.INTERACTIONS & 0.07 \\
\hline GAB1.SIGNALOSOME & 0.07 \\
\hline WP2583.T.CELL.RECEPTOR.AND.CO.STIMULATORY.SIGNALING & 0.07 \\
\hline CAP.DEPENDENT.TRANSLATION.INITIATION & 0.07 \\
\hline WP2710.NONSENSE.MEDIATED.DECAY & 0.08 \\
\hline ROLE.OF.LAT2.NTAL.LAB.ON.CALCIUM.MOBILIZATION & 0.08 \\
\hline DOWNREGULATION.OF.SMAD2.3.SMAD4.TRANSCRIPTIONAL.ACTIVITY & 0.08 \\
\hline FC.EPSILON.RECEPTOR.FCERI.SIGNALING & 0.08 \\
\hline WP1868.NETRIN.1.SIGNALING & 0.08 \\
\hline WP2737.SRP.DEPENDENT.COTRANSLATIONAL.PROTEIN.TARGETING.TO.MEMBRANE & 0.08 \\
\hline WP1911.SIGNALING.BY.FGFR & 0.08 \\
\hline EUKARYOTIC.TRANSLATION.INITIATION & 0.08 \\
\hline WP2804.HAIR.FOLLICLE.DEVELOPMENT.INDUCTION.PART.1.OF.3. & 0.08 \\
\hline BIOC_IGF1PATHWAY & 0.08 \\
\hline SRP.DEPENDENT.COTRANSLATIONAL.PROTEIN.TARGETING.TO.MEMBRANE & 0.08 \\
\hline PI3K.CASCADE & 0.09 \\
\hline WP2653.PIP3.ACTIVATES.AKT.SIGNALING & 0.09 \\
\hline NONSENSE.MEDIATED.DECAY.NMD.ENHANCED.BY.THE.EXON.JUNCTION.COMPLEX.EJC. & 0.10 \\
\hline BASIGIN.INTERACTIONS & 0.10 \\
\hline
\end{tabular}

Supplementary Table S3-5. Underrepresented gene sets when comparing the response to fish-oil supplementation in APOE4 carriers to non-carriers using GSEA

\begin{tabular}{ll}
\hline NAME & FDR Q-value \\
\hline INTERFERON.ALPHA.BETA.SIGNALING & 0.00 \\
WP1835.INTERFERON.ALPHA.BETA.SIGNALING & 0.00 \\
INTERFERON.SIGNALING & 0.00 \\
WP619.TYPE.II.INTERFERON.SIGNALING.IFNG. & 0.00 \\
INTERFERON.GAMMA.SIGNALING & 0.00 \\
TRAF6.MEDIATED.IRF7.ACTIVATION & 0.01 \\
HSA05162 & 0.01 \\
HSA05164 & 0.02 \\
CYTOKINE.SIGNALING.IN.IMMUNE.SYSTEM & 0.02 \\
BIOC_PROTEASOMEPATHWAY & 0.02 \\
HSA05168 & 0.02
\end{tabular}


APOE4 and fish-oil

\begin{tabular}{ll}
\hline NAME & FDR Q-value \\
\hline HSA04623 & 0.02 \\
WP2796.CLASS.I.MHC.MEDIATED.ANTIGEN.PROCESSING.AMP.PRESENTATION & 0.03 \\
WP183.PROTEASOME.DEGRADATION & 0.03 \\
HSA05160 & 0.04 \\
WP2784.BINDING.AND.UPTAKE.OF.LIGANDS.BY.SCAVENGER.RECEPTORS & 0.05 \\
AUF1.HNRNP.D0.DESTABILIZES.MRNA & 0.05 \\
ANTIGEN.PROCESSING.CROSS.PRESENTATION & 0.05 \\
RIG.I.MDA5.MEDIATED.INDUCTION.OF.IFN.ALPHA.BETA.PATHWAYS & 0.05 \\
VIF.MEDIATED.DEGRADATION.OF.APOBEC3G & 0.06 \\
ER.PHAGOSOME.PATHWAY & 0.06 \\
WP2794.CYTOSOLIC.SENSORS.OF.PATHOGEN.ASSOCIATED.DNA. & 0.06 \\
CDK.MEDIATED.PHOSPHORYLATION.AND.REMOVAL.OF.CDC6 & 0.07 \\
HSA03050 & 0.07 \\
WP455.GPCRS.CLASS.A.RHODOPSIN.LIKE & 0.07 \\
WP1904.RIG.I.MDA5.MEDIATED.INDUCTION.OF.IFN.ALPHA.BETA.PATHWAYS & 0.09 \\
CROSS.PRESENTATION.OF.SOLUBLE.EXOGENOUS.ANTIGENS.ENDOSOMES. & 0.10 \\
HSA04612 & 0.10 \\
GLI3.IS.PROCESSED.TO.GLI3R.BY.THE.PROTEASOME & 0.10 \\
\hline
\end{tabular}




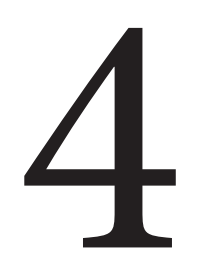

\section{Dietary medium-chain saturated fatty acids induce gene expression of energy metabolism- related pathways in adipose tissue of abdominally obese subjects}

Juri Matualatupauw, Mette Bohl, Søren Gregersen, Kjeld Hermansen and Lydia Afman 


\section{Abstract}

\section{Background}

Dietary medium-chain saturated fatty acids (MC-SFAs) have been shown to reduce total body fat. Previously, we showed that MC-SFAs prevent body fat accumulation, despite weight gain. Here, we aim to explore potential molecular mechanisms underlying the protective effect of MC-SFAs on body fat gain.

\section{Methods}

The DairyHealth study examined the long-term effects of milk protein and milk fat with a low or high content of MC-SFA. In this 12 week, randomized, double-blind, diet intervention study, participants consumed 60g milk protein (whey or casein) and 63g milk fat (high MCSFA or low MC-SFA) daily in a 2 by 2 factorial design. We used microarrays to measure whole genome gene expression changes in subcutaneous adipose tissue in a subpopulation of 12 participants, 6 in the low MC-SFA + casein group and 6 in the high MC-SFA + casein group. Gene expression of several genes that were found to be changed by MC-SFAs was confirmed in the full study population using qPCR.

\section{Results}

High MC-SFA resulted in an upregulation of gene expression related to citric acid cycle, oxidative phosphorylation and a downregulation of gene expression related to complement system and inflammation.

\section{Conclusions}

We hypothesize that the beneficial effects of MC-SFAs on prevention of fat accumulation are mediated via increased gene expression related to energy metabolism in the adipose tissue. Decreases in inflammation-related gene expression may have beneficial effects in relation to cardiometabolic diseases. 


\section{Introduction}

Medium-chain saturated fatty acids (MC-SFAs) are present in several types of foods including dairy products. Two recent meta-analyses show beneficial effects of intake of MC-SFAs on body weight and body composition and conclude that consumption of medium-chain triglycerides in exchange of long-chain triglycerides as part of a diet results in a reduction in total,[1] visceral and subcutaneous body fat.[2]

In contrast to long-chain saturated fatty acids, MC-SFAs from foods are not incorporated into chylomicrons, but are transported directly to the liver as free fatty acids. In the liver, most of the MC-SFAs are oxidized directly[3]. This is thought to be the cause of the postprandial increase in energy expenditure observed after consumption of MC-SFAs.[4, 5] A smaller fraction of the MC-SFAs is distributed via the circulation to peripheral tissues such as the adipose tissue. $[3,6]$ In humans, MC-SFAs have been shown to be present in adipose tissue after a diet enriched in MC-SFAs.[7] Moreover, in rats, medium chain fatty acids (MCFAs) from the diet were shown to be incorporated into adipose tissue triglycerides. [8] Furthermore, in this rat study a reduction in fat mass and a downregulation of adipogenic genes, including peroxisome proliferator activated receptor gamma $(\operatorname{PPAR} \gamma)$ and CCAAT/enhancer-binding protein alpha (C/EBPa) was observed after the MCFA-diet compared to an isocaloric highfat control diet. Moreover, in cultured murine and human adipocytes, incubation with the MC-SFA octanoic acid, inhibited adipogenesis and expression of adipogenic genes.[9] Taken together, these studies indicate that the effects dietary MC-SFAs on body weight and body composition are not only achieved by increased fatty acid oxidation in the liver, but suggest that MC-SFAs can affect adipose tissue as well. However, knowledge on the precise effects of dietary MC-SFAs on adipose tissue in humans is lacking. To investigate the effects of MC-SFAs on human adipose tissue, we studied whole genome gene expression profiles on subcutaneous adipose tissue biopsies of the DairyHealth study. This dietary intervention study examined the long-term effects of intake of milk fat with a low or high content of MCSFAs and milk protein in abdominally obese participants.[10] In this study, all intervention groups increased their body weight, though an increase in total body fat was only observed in the low MC-SFAs groups. The high MC-SFA groups were protected against this body fat gain and these individuals displayed a gain in lean body mass.[11]

We aim to increase our understanding of the effects of MC-SFAs on the adipose tissue that may explain the observed differences in body fat accumulation. To do this, we investigated the 
effects of a 12-week high MC-SFA versus a low MC-SFA diet on subcutaneous adipose tissue gene expression profiles.

\section{Materials and Methods}

\section{Participants}

Participant recruitment procedures were described in detail previously.[10] In short, participants were eligible for inclusion if $\geq 18 \mathrm{y}$ of age, had an abdominal circumference of $\geq 80 \mathrm{~cm}$ for women or $\geq 94 \mathrm{~cm}$ for men, and were weight stabile for $\geq 3$ months. Exclusion criteria were diagnosis with diabetes, pregnancy, lactating, or severe cardiovascular, renal, endocrine, or psychiatric disease. Fifty-two participants completed the study. Adipose tissue samples were not collected from three participants ( 2 because of bleeding and 1refrained from the post-intervention biopsy) and adipose tissue samples from two participants are missing because of early defrosting.

\section{Study design}

This study is a secondary analysis of a randomized, parallel-controlled, double-blinded, 12 -week diet intervention study that was designed to examine the effects of milk fat, with a high or low MC-SFA content, and of milk protein, whey or casein, on post-prandial lipemia. Design of the original study was described in detail previously.[10] Briefly, 52 participants were randomized in a $2 \times 2$ factorial design to one of 4 diets: high MC-SFA + casein, low MCSFA + casein, high MC-SFA + whey, and low MC-SFA + whey. In this paper, we examined the effects of MC-SFAs on whole genome gene expression in subcutaneous adipose tissue in a subpopulation of 12 subjects, 6 in the high MC-SFA + casein group and 6 in the low MCSFA + casein groups. We chose to perform the microarray analysis in the casein groups, as the MC-SFA-induced prevention of body fat accumulation was most pronounced in these groups.[11] To validate findings of the microarray analyses and to examine gene expression changes in the whey groups, we performed targeted quantitative real-time polymerase chain reaction (qPCR) measurements on all adipose tissue samples.

All participants gave written informed consent to participate in the study and the study protocol was approved by the Central Denmark Region Committees on Health Research Ethics. The study was registered at clinicaltrials.gov as NCT01472666. 


\section{Dietary intervention}

Participants consumed food products containing $63 \mathrm{~g}$ of milk fat and $60 \mathrm{~g}$ of milk protein daily. The milk fat contained 8.5g and 6.9g of MC-SFAs in the high MC-SFA and the low MC-SFA groups respectively. Protein was either whey or casein. The milk fats were produced at the Danish Cattle Research Center (Foulum, Denmark) by feeding cattle high- or low-fat diets in order to produce low and high MC-SFA- containing milk respectively (16). Butter was produced from these two types of milk and used as spread and in the production of rolls and cakes. Protein powder containing whey (Lacprodan DI-9224) or casein (Miprodan 30) was provided by Arla Foods Ingredients Group P/S (Viby J, Denmark).

The daily food products were 2 rolls, 1 cake, and $25 \mathrm{~g}$ of butter (corresponding to a total of $63 \mathrm{~g}$ of milk fat/day) and two protein shakes (a total of $60 \mathrm{~g} /$ day of protein). Daily energy intake from the test products was 6,200 kJ with 42 energy percentage (E\%) as fat, $37 \mathrm{E} \%$ as carbohydrate, and $21 \mathrm{E} \%$ as protein.

A clinical dietician used the participants' dietary registrations and their calculated energy requirement to instruct the participants on how to incorporate the dietary supplementation into their habitual diet.

\section{Dual-energy X-ray absorption scan}

Whole body composition, total body fat, android fat percentage, gynoid fat percentage, and lean mass were measured by DEXA scan (Hologic Discovery A scanner, serial nr. 83986, Hologic Inc., Danbury, USA). To generate DEXA scan output we used the Hologic software Apex (Version 13.1.1:3).

\section{Fat biopsies}

Before and after intervention, fat biopsy specimens were taken from the abdominal subcutaneous adipose tissue. The biopsy specimens were taken with Bergstrom needle through a small skin incision under local analgesia with $10-15 \mathrm{~mL}$ lidocaine $(20 \mathrm{~g} / \mathrm{L})$. The adipose tissue was cleaned for blood with sterile saline, snap-frozen in liquid nitrogen, and stored at $-80^{\circ} \mathrm{C}$.

\section{RNA isolation}

Adipose tissue samples were homogenized in TRIzol $^{\oplus}$ Reagent (Invitrogen, Breda, the Netherlands). RNA was extracted using chloroform and purified using Qiagen RNeasy Mini 
Kit (Qaigen, Venlo, the Netherlands). RNA concentration was measured on a Nanodrop ND 1000 spectrophotometer (Nanodrop) and integrity was assessed using an Agilent 2100 Bioanalyzer (Agilent). Samples with RIN values above 7.5 were considered suitable for microarray and qPCR analysis.

\section{Microarray processing}

Six subjects from the high MC-SFA + casein group and six from the low MC-SFA + casein group were selected for microarray analysis, so that each group contained the same number of males/females and subjects with the metabolic syndrome. Microarrays were performed before and after the intervention, resulting in a total of 24 microarrays. Total RNA was labelled using a one-cycle cDNA labeling kit (MessageAmp II-Biotin Enhanced Kit; Ambion) and hybridized to Genechip Human Gene 1.1 ST arrays, containing 803487 probes (Affymetrix, Santa Clara, CA). Sample labelling, hybridization to chips and image scanning was performed according to the manufacturers' instructions.

\section{Microarray analysis}

Microarray analysis was performed using MADMAX pipeline for statistical analysis of microarray data.[12] A custom annotation was used based on reorganized oligonucleotide probes, which combines all individual probes for a gene (custom CDF[13]). This resulted in a total of 19,621 genes. Expression values were calculated using robust multichip average method, which includes quantile normalization.[14] Probe sets with unlogged expression values higher than 20 on at least 1 array were selected for further statistical analysis. Significant differences in expression were assessed using limma.[15] For all comparisons genes were defined as significantly different when the P-value was $<0.05$. The response to the intervention in the high MC-SFA + casein and the low MC-SFA + casein groups was determined using paired t-tests with Bayesian correction as applied in limma. Differences in gene expression changes between the two groups were calculated from the individual log ratios and was calculated as the response to high MC-SFA + casein compared to the response to low MC-SFA + casein using unpaired t-tests with Bayesian correction as implemented in limma. Microarray data are registered as GSE87382 in the Gene Expression Omnibus.

\section{Pathway analyses}

We performed pre-ranked gene set enrichment analysis (GSEA; http://www.broad.mit. edu/gsea).[16] Briefly, genes were ranked based on the t-statistic and analyzed for over- or 
underrepresentation in predefined gene sets. Gene sets were derived from Biocarta, KEGG, Reactome and Wikipathways databases. To increase the strength of our findings, we used a relatively strict cut-off of FDR $\mathrm{q}<0.1$. The significant gene sets were visualized in Cytoscape using Enrichment Map.[17]

\section{Upstream regulator analysis}

Upstream transcription regulators analyses were performed using QIAGEN's Ingenuity Pathway Analysis (IPA, QIAGEN Redwood City, www.qiagen.com/ingenuity). A P-value cutoff of 0.05 was used determine differentially expressed genes. Upstream regulators were defined significant when the P-value was below 0.05 and the $\mathrm{z}$ score was $>2$ or $<-2$. Upstream regulators with $\mathrm{z}$ scores $>2$ were considered activated and regulators with $\mathrm{z}$ scores $<2$ were considered inhibited.

\section{QPCR}

To confirm microarray results, we performed qPCR in samples in the full study population. RNA was reverse transcribed using a cDNA synthesis kit (First Strand cDNA Synthesis kit, Thermo Scientific, Leusden, the Netherlands) and analyzed by qPCR (SensiMix SYBR No-ROX, Bioline, London, UK) on a CFX384 Real-Time System (C1000 Thermal Cycler, Biorad, Veenendaal, The Netherlands). qPCR data were normalized using ACTB and PPIA as housekeeping genes. A calibration curve was created from 1:10 serial dilutions of a pool of all samples. Using this calibration curve, relative starting quantities were determined for each gene in each sample. Expression of genes of interested were normalized by calculating log2ratios of relative starting quantity between gene of interest and the housekeeping genes. Genes for qPCR were selected from the differentially expressed pathways in the microarray analysis using several criteria. Genes were required to have P-value $<0.05$ in the comparison between the high MC-SFA + casein and the low MC-SFA + casein group, have a raw expression value above 200 (which leads to ct-values < 34), and have primers available in the PrimerBank database.[18] Primer sequences are shown in Supplementary Table S4-1.

\section{Statistical analysis}

Differences in baseline characteristics were analyzed using independent t-tests using IBM SPSS Statistics, version 22.0.0.1. Body weight changes, body composition changes and qPCR data were analyzed using one sample t-tests and independent t-tests with GraphPad Prism version 5.04. For all analyses P-values $<0.05$ were considered statistically significant. 


\section{Results}

\section{Participant characteristics}

In total, 52 subjects completed the original study. Baseline characteristics of the total population have been reported previously.[10] In this study, we performed microarrays in adipose tissue samples of 6 subjects in the high MC-SFA + casein group and 6 subjects in the low MC-SFA + casein group. Baseline characteristics of these subgroups are shown in Supplementary Table S4-2.

\section{Change in body weight and composition}

Subjects consumed butter containing 63g of milk fat (high or low in MC-SFAs) and 60g of protein (whey or casein) daily for twelve weeks. Changes in body weight and composition for the full study population were reported in detail previously.[11] Briefly, body weight was increased after intervention, though there was no difference between groups or difference depending on MC-SFA content and protein type. Total fat percentage and gynoid fat percentage increased when consuming the butter low in MC-SFA, whereas lean mass increased in the participants consuming the butter high in MC-SFA. The changes in body weight and composition in our microarray subgroups were similar to those in the full study population (Figure 4-1 and [11]). In our subgroups, body weight increased significantly in the low MC-SFA + casein group only $(\mathrm{P}=0.024)$, though body weight changes were not different compared to the high MC-SFA + casein group. Furthermore, only the low MC-SFA + casein group showed an increased total body $(\mathrm{P}=0.045)$ and android fat percentage $(\mathrm{P}=$ $0.028)$. Changes in total body and android fat percentage were also different between the two groups ( $\mathrm{P}=0.028$ and $\mathrm{P}=0.018$, respectively).

\section{Differentially expressed genes}

We compared whole genome gene expression profiles after intervention to those before intervention in the high MC-SFA + casein and the low MC-SFA + casein groups. To examine the effects of MC-SFAs on adipose tissue, we compared the changes in gene expression between the high MC-SFA + casein and the low MC-SFA + casein group and found 986 genes to be differentially changed between these two groups (Figure 4-2). Principal component analysis on the changes in expression of these 986 genes revealed a clear separation between the low MC-SFA + casein and high MC-SFA + casein groups (Supplementary Figure S4-1). 


\section{Pathway analysis}

To gain further insight into the biological processes affected by MC-SFAs, we performed GSEA for the difference in response between both groups and for the response within the groups. GSEA showed that 68 gene sets were significantly upregulated and 31 were significantly downregulated when comparing the response on high MC-SFA to the response on low MCSFA (Supplementary Table S4-3). These gene sets are clustered on overlapping genes and are visualized in Supplementary Figure S4-2 and summarized in Table 4-1. Gene sets related to citric acid cycle, oxidative phosphorylation and adipogenesis were significantly upregulated in the high MC-SFA + casein group and significantly downregulated in the low MC-SFA + casein group. Gene sets related to inflammation and complement cascade were significantly downregulated by intake of high MC-SFA + casein and significantly upregulated after intake of low MC-SFA + casein. For each of these gene set clusters, we selected the genes that were significantly differentially changed when comparing the high MC-SFA + casein group and the low MC-SFA + casein group. Individual expression changes upon both interventions of these genes are visualized in Figure 4-3.

\section{Upstream regulator analysis}

To examine potential regulators of the differentially expressed genes, we performed Ingenuity upstream regulator analysis (Table 4-2). Two transcription factors regulating lipid metabolism and oxidative phosphorylation, PPARa and PPARGC1a, were predicted to be activated by MC-SFAs. Likewise, transcriptional regulators involved in inflammation STAT6, NFKB1 and IKBKB were predicted to be inhibited by MC-SFAs.

\section{QPCR validation of the microarray findings}

To examine the expression changes of several selected genes of the microarray analysis in the total study population, we performed qPCR. Genes were selected from the inflammation, complement cascade, citric acid cycle, oxidative phosphorylation and adipogenesis pathways. Gene expression changes in the 4 intervention groups are shown in Figure 4-4. Expression changes of ACOX1, SLC25A10, SDHB, and IDH3B were significant for MC-SFA independent of protein type. Within the casein groups we observed changes in similar directions as the microarray results for both high and low MC-SFA groups for all genes, except CFH. An MCSFA and protein interaction effect was observed for the SLC25A10, DLAT and SDHB genes, indicating differences in response to the MC-SFAs between the casein and the whey groups. 


\section{Discussion}

In this study, we showed that 12 weeks consumption of butter high in MC-SFA + casein in subjects with central obesity had marked effects on subcutaneous adipose tissue gene expression profiles compared to consumption of butter low in MC-SFA + casein. MC-SFAs induced gene expression in energy metabolism-related pathways such as oxidative phosphorylation and citric acid cycle as well as lipid metabolism-related pathways. Furthermore, MCSFAs decreased pathways related to inflammation and complement system. These effects were observed with a simultaneous protection against body fat accumulation and provide valuable insight into the potential mechanism behind the beneficial effects of MC-SFAs on fat accumulation and adipose tissue metabolism.

The increase in gene expression in the citric acid cycle and oxidative phosphorylation pathways in the high MC-SFA group could be indicative of an increase in oxidative energy metabolism in the adipose tissue. Moreover, PPARGCla, which is the key regulator of the oxidative phosphorylation pathway,[19] was identified as a likely upstream regulator pointing to a potential activation of this pathway via this transcription factor. To our knowledge, these effects of MC-SFAs on energy metabolism pathways in the adipose tissue are novel findings. In the liver, dietary medium chain fatty acids do not require carnitine to reach the mitochondria, but passively cross the mitochondrial membrane and are beta-oxidized rapidly. Subsequently, the produced acetyl-CoA enters the citric acid cycle and is oxidized [3], resulting in a postprandial increase in energy expenditure after consumption of MC-SFAs $(4,5)$. MC-SFAs have been shown to reach the adipose tissue, $[7,8]$ where they may be rapidly beta-oxidized. We observed an MC-SFA-induced increase in expression of ACOX1, which codes for an enzyme involved in fatty acid beta-oxidation. ACOX1 is a target gene of PPARa, an important regulator of fatty acid B-oxidation,[20] which was identified as a predicted upstream regulator in our analysis. In line with this, in a study in rats, fatty acid oxidation measured as carnitine palmitoyl transferase activity was higher when the animals were fed triglycerides containing medium- and long-chain fatty acids compared to triglycerides containing long chain fatty acids only.[21] In these rats, this change in fatty acid oxidation was combined with a concurrent decrease in body fat accumulation. Another interesting PPARa-target gene of which the expression was upregulated upon high MC-SFA is APOC3. APOC3 inhibits LPL activity resulting in a decreased triglyceride hydrolysis and hence a reduced uptake of fatty acids in the adipose tissue. The potential MC-SFAs-induced increase in fatty acid oxidation, increase in energy metabolism and decrease in uptake of fatty acids in the adipose tissue may 
result in a reduced fat storage which may explain the observed preventive effect of MC-SFAs on body fat accumulation.

We did not observe a downregulation in expression of genes involved in adipogenesis upon MC-SFA, which is in contrast with findings from rat studies. Sinahara et al.[21] reported a suppressing effect of triglycerides containing medium and long-chain fatty acids compared to triglycerides containing long chain fatty acids on adipogenesis in adipose tissue in rats, as shown by lower fatty acid synthase (FAS) enzyme activity, though gene expression of FAS was not affected. Similarly, another study in rats showed a downregulation in expression of the adipogenic genes PPAR $\gamma$ and $\mathrm{C} / \mathrm{EBPa}$ in adipose tissue in response to medium chain triglycerides.[8] We did not observe changes in adipogenesis-related gene expression, including FAS, PPAR $\gamma$ and C/EBPa, though it is still possible that FAS enzyme activity may have been increased. Taken together, based on these findings, we hypothesize that the preventive effects of MC-SFAs on body fat accumulation were not caused by a decrease in adipogenesis.

The MC-SFA-induced downregulation in pathways related to inflammation, and especially in genes related to the complement system may point towards a decreased inflammatory state of the adipose tissue. Complement components are produced in adipose tissue and are involved in both adipose tissue inflammation as well as adipose tissue metabolism.[22, 23] The MC-SFAs-induced decrease in inflammation-related pathways may be beneficial considering the deleterious role of inflammatory pathways in the adipose tissue in initiating and sustaining the low-grade inflammatory state that is associated with obesity.[24] To our knowledge, no previous studies have observed effects of MC-SFAs on inflammation-related gene expression in adipose tissue in humans. In mice, a high-fat diet supplemented with medium chain triglycerides resulted in significantly lower serum IL-6 levels and higher IL-10 levels compared to an isocaloric high-fat diet. In addition, hepatic activation of NF- $\kappa \mathrm{B}$ and p38 MAPK was reduced.[25] Our upstream regulator analysis identified NF- $\kappa B$ and IKBKB as potential inhibited upstream regulators in the adipose tissue, indicating that an increased MC-SFA intake may inhibit the NF-kB pathway.

The changes in inflammation-related gene expression were not paralleled by a reduction in plasma inflammatory markers as no changes in IL-6, IL-1RA, high-sensitive CRP, adiponectin and MCP-1 between the four groups were found in this study.[26] It should be noted that gene expression of none of these markers were altered in our microarray analyses. However, gene expression changes in the adipose tissue may be one of the first changes induced by dietary 
interventions,[27] and a reflection of this on circulating inflammation markers may only develop after a prolonged exposure.

The qPCR analyses confirmed the described effects of MC-SFAs on gene expression in the full casein groups. However, in the two whey groups, we did not observe the same effect of MCSFA. Accordingly, the changes in body fat percentage also appear less pronounced in the whey groups.[11] One study in mice showed that whey reduced high-fat diet-induced body weight and body fat gain compared to casein.[28] They used qPCR to measure gene expression of a limited number of genes in adipose tissue and, inconsistent with our findings, found no effects of whey protein on expression of genes related to energy metabolism, adipogenesis or inflammation. It should be noted, that the genes reported in the mice study were different from the genes observed to be differentially expressed in our study. Furthermore, differences between species, length of the dietary intervention and dietary composition may also explain the differential findings between this mouse study and our study.

We have used a relatively small number of subjects for the microarray analysis with 6 subjects in the high MC-SFA + casein group and 6 subjects in the low MC-SFA + casein group. Because of this, we found no differentially expressed genes when using FDR q-value $<0.05$ as significance cutoff. Instead, we used a p-value cut-off of 0.05 for selecting the differentially expressed genes. For this reason, we focused this discussion mostly on the GSEA analyses and less on individual genes. To increase the strength of our GSEA findings, we used a strict cutoff of FDR $q<0.1$. Despite the small sample size and the use of stringent cut of values, we still observed a robust gene expression pathway effect of 12 weeks of supplementation with butter high in MC-SFAs. Furthermore, we used qPCR to validate our findings for several genes in the total study population and found that our microarray findings in the high or low MC-SFA + casein groups were largely confirmed in the full groups.

In the DairyHealth study,[10] subjects with abdominal obesity were protected from an increase in body fat accumulation by a 12 week high MC-SFA dietary intervention compared to low MC-SFA.[11] We investigated the underlying mechanism behind this protective effect of MCSFAs on adipose tissue using whole genome gene expression analyses. Our findings on gene expression point towards an MC-SFA-induced increase in energy metabolism, an increase in fatty acid oxidation and a decrease in fatty acid uptake in adipose tissue that is potentially responsible for the protective effect on body fat accumulation. In addition, a decrease in expression of genes involved in inflammation and complement system pathways may reflect a decreased inflammatory state of the adipose tissue. Taken together, these findings indicate 
that the beneficial effects of MC-SFAs are not only achieved in the liver, but suggest that the adipose tissue could play a key role in mediating the effects of MC-SFAs as well.

\section{References}

1. Bueno, N.B., et al., Dietary medium-chain triacylglycerols versus long-chain triacylglycerols for body composition in adults: systematic review and meta-analysis of randomized controlled trials. J Am Coll Nutr, 2015. 34(2): p. 175-83.

2. Mumme, K. and W. Stonehouse, Effects of medium-chain triglycerides on weight loss and body composition: a meta-analysis of randomized controlled trials. J Acad Nutr Diet, 2015. 115(2): p. 249-63.

3. Bach, A.C. and V.K. Babayan, Medium-chain triglycerides: an update. Am J Clin Nutr, 1982. 36(5): p. 950-62.

4. Hill, J.O., et al., Thermogenesis in humans during overfeeding with medium-chain triglycerides. Metabolism, 1989. 38(7): p. 641-8.

5. Nosaka, N., et al., Effects of margarine containing medium-chain triacylglycerols on body fat reduction in humans. J Atheroscler Thromb, 2003. 10(5): p. 290-8.

6. Marten, B., M. Pfeuffer, and J. Schrezenmeir, Medium-chain triglycerides. International Dairy Journal, 2006. 16(11): p. 1374-1382.

7. Sarda, P., et al., Storage of medium-chain triglycerides in adipose tissue of orally fed infants. Am J Clin Nutr, 1987. 45(2): p. 399-405.

8. Han, J.R., et al., Medium-chain oil reduces fat mass and down-regulates expression of adipogenic genes in rats. Obesity Research, 2003. 11(6): p. 734-744.

9. Guo, W., et al., Octanoate inhibits triglyceride synthesis in 3T3-L1 and human adipocytes. J Nutr, 2003. 133(8): p. 2512-8.

10. Bohl, M., et al., Dairy proteins, dairy lipids, and postprandial lipemia in persons with abdominal obesity (DairyHealth): a 12-wk, randomized, parallel-controlled, double-blinded, diet intervention study. Am J Clin Nutr, 2015. 101(4): p. 870-8.

11. Bohl, M., et al., The effects of proteins and medium-chain fatty acids from milk on body composition, insulin sensitivity and blood pressure in abdominally obese adults. 2016.

12. Lin, K., et al., MADMAX - Management and analysis database for multiple omics experiments. J Integr Bioinform, 2011. 8(2): p. 160.

13. Dai, M., et al., Evolving gene/transcript definitions significantly alter the interpretation of GeneChip data. Nucleic Acids Res, 2005. 33(20): p. e175.

14. Irizarry, R.A., et al., Summaries of Affymetrix GeneChip probe level data. Nucleic Acids Res, 2003. 31(4): p. e15.

15. Ritchie, M.E., et al., limma powers differential expression analyses for RNA-sequencing and microarray studies. Nucleic Acids Res, 2015. 43(7): p. e47.

16. Subramanian, A., et al., Gene set enrichment analysis: a knowledge-based approach for interpreting genomewide expression profiles. Proc Natl Acad Sci U S A, 2005. 102(43): p. 15545-50.

17. Merico, D., et al., Enrichment map: a network-based method for gene-set enrichment visualization and interpretation. PLoS One, 2010. 5(11): p. e13984.

18. Wang, X. and B. Seed, A PCR primer bank for quantitative gene expression analysis. Nucleic Acids Res, 2003. 31(24): p. e154.

19. $\mathrm{Wu}, \mathrm{Z}$., et al., Mechanisms controlling mitochondrial biogenesis and respiration through the thermogenic 
coactivator PGC-1. Cell, 1999. 98(1): p. 115-24.

20. Mandard, S., M. Muller, and S. Kersten, Peroxisome proliferator-activated receptor alpha target genes. Cell Mol Life Sci, 2004. 61(4): p. 393-416.

21. Shinohara, H., et al., Randomly interesterified triacylglycerol containing medium- and long-chain fatty acids stimulates fatty acid metabolism in white adipose tissue of rats. Biosci Biotechnol Biochem, 2006. 70(12): p. 2919-26.

22. Barbu, A., et al., The role of complement factor C3 in lipid metabolism. Mol Immunol, 2015. 67(1): p. 101-7.

23. Hertle, E., C.D. Stehouwer, and M.M. van Greevenbroek, The complement system in human cardiometabolic disease. Mol Immunol, 2014. 61(2): p. 135-48.

24. Tilg, H. and A.R. Moschen, Adipocytokines: mediators linking adipose tissue, inflammation and immunity. Nat Rev Immunol, 2006. 6(10): p. 772-83.

25. Geng, S., et al., Medium-chain triglyceride ameliorates insulin resistance and inflammation in high fat dietinduced obese mice. Eur J Nutr, 2015.

26. Bohl, M., et al., Whey and Casein Proteins and Medium-Chain Saturated Fatty Acids from Milk Do Not Increase Low-Grade Inflammation in Abdominally Obese Adults. Rev Diabet Stud, 2016.

27. van Dijk, S.J., et al., A saturated fatty acid-rich diet induces an obesity-linked proinflammatory gene expression profile in adipose tissue of subjects at risk of metabolic syndrome. Am J Clin Nutr, 2009. 90(6): p. 1656-64.

28. McAllan, L., et al., Whey protein isolate counteracts the effects of a high-fat diet on energy intake and hypothalamic and adipose tissue expression of energy balance-related genes. Br J Nutr, 2013. 110(11): p. 211426. 


\section{Tables}

Table 4-1. Results of the gene set enrichment analysis.

\begin{tabular}{lcc}
\hline Gene set cluster & High MC-SFA + casein & Low MC-SFA + casein \\
\hline Citric acid cycle & $\uparrow$ & $\downarrow$ \\
Oxidative phosphorylation & $\uparrow$ & $\downarrow$ \\
Lipid metabolism & $\uparrow$ & $\downarrow$ \\
Inflammation & $\downarrow$ & $\downarrow$ \\
Complement system & $\downarrow$ & $\downarrow$ \\
\hline
\end{tabular}

a Gene sets differentially changed in the high MC-SFA + casein compared to the low MC-SFA + casein group were clustered and visualized using Enrichment Map in Cytoscape (Supplementary Figure S4-1). From this, gene set clusters were selected and changes in expression in each group are shown. Full GSEA results are shown in Supplementary Table S4-3. $\uparrow$, significantly upregulated; $\downarrow$, significantly downregulated.

Table 4-2. Top 10 potential upstream regulators explaining the differences in gene expression changes between the high MC-SFA + casein and the low MC-SFA + casein groups. ${ }^{a}$

\begin{tabular}{lllll}
\hline $\begin{array}{l}\text { Upstream } \\
\text { Regulator }\end{array}$ & Molecule Type & $\begin{array}{l}\text { Predicted } \\
\text { Activation }\end{array}$ & $\begin{array}{l}\text { Activation } \\
\text { z-score }\end{array}$ & p-value of overlap \\
\hline PPARA & ligand-dependent nuclear receptor & Activated & 2.049 & $4.28 \mathrm{E}-04$ \\
ESR1 & ligand-dependent nuclear receptor & Inhibited & -3.285 & $9.53 \mathrm{E}-04$ \\
PDLIM2 & other & Activated & 2.111 & $1.27 \mathrm{E}-03$ \\
PPARGC1A & transcription regulator & Activated & 2.24 & $3.47 \mathrm{E}-03$ \\
NFKB1 & transcription regulator & Inhibited & -2.377 & $2.03 \mathrm{E}-03$ \\
STAT6 & transcription regulator & Inhibited & -2.35 & $3.18 \mathrm{E}-03$ \\
MITF & transcription regulator & Inhibited & -3.727 & $3.78 \mathrm{E}-03$ \\
IKBKB & kinase & Inhibited & -2.358 & $3.88 \mathrm{E}-03$ \\
BID & other & Inhibited & -2.000 & $5.29 \mathrm{E}-03$ \\
HRG & other & Inhibited & -2.000 & $7.85 \mathrm{E}-03$ \\
\hline
\end{tabular}

a The 10 regulators with the lowest P-value and a $z$-score $>2$ or $<-2$ are shown for the difference in gene expression changes in response to a 12-week dietary intervention containing high MC-SFA + casein compared to low MC-SFA + casein. Z-scores predict the activation state of the regulator and are based on the gene expression of its downstream genes. Upstream regulators with $z$ scores $>2$ are considered activated and regulators with $z$-scores $<2$ are considered inhibited. P-values of overlap measure the significance of the overlap between the differentially expressed genes and the genes reported to be regulated by the upstream regulator and are calculated using Fisher's exact test. 


\section{Figures}

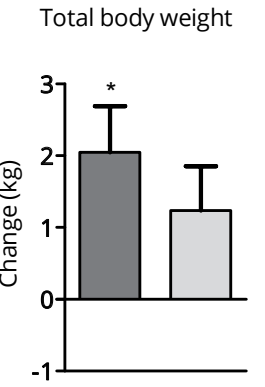

Android fat percentage
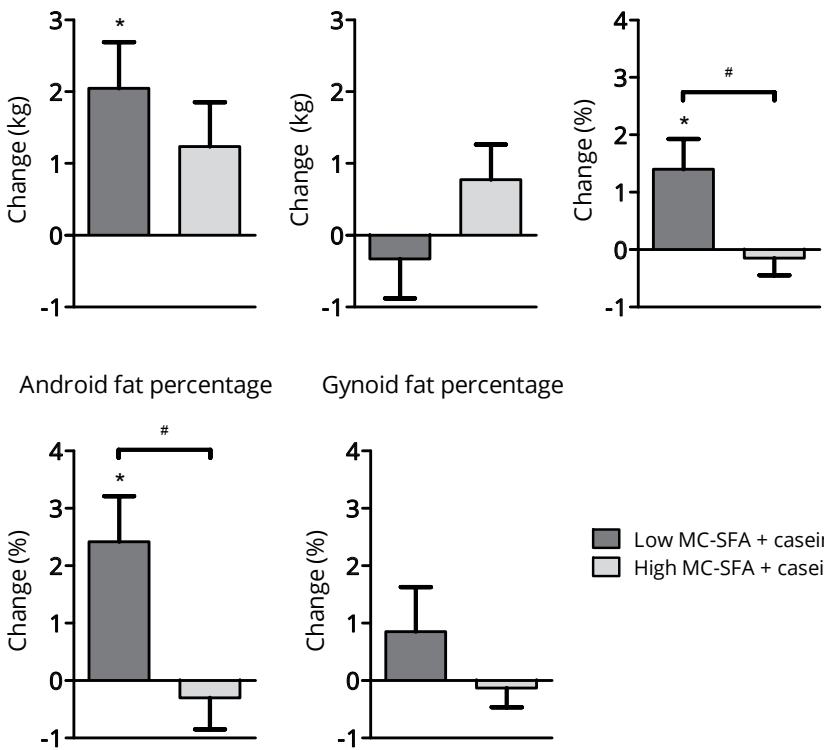

Gynoid fat percentage

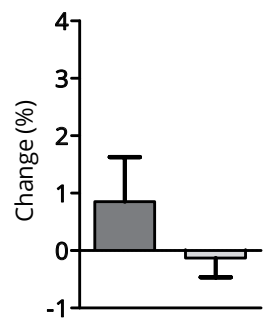

$\square$ Low MC-SFA + casein
$\square$ High MC-SFA + casein

Figure 4-1. Change in total body weight, lean body weight, total fat percentage, android fat percentage and gynoid fat percentage in the subgroups (high MC-SFA + casein and low MC-SFA + casein) selected for the microarray analysis in this study.

Data were analysed using paired t-tests (within group changes) and independent t-tests (between groups differences in changes) and are presented as mean \pm SEM. $\mathrm{P}<0.05$ is considered significant. *Significantly different change within group. \# Significantly different change between groups. Data for the full study population was previously published in Bohl et al.[11]. 
Effects of medium-chain saturated fatty acids in adipose tissue

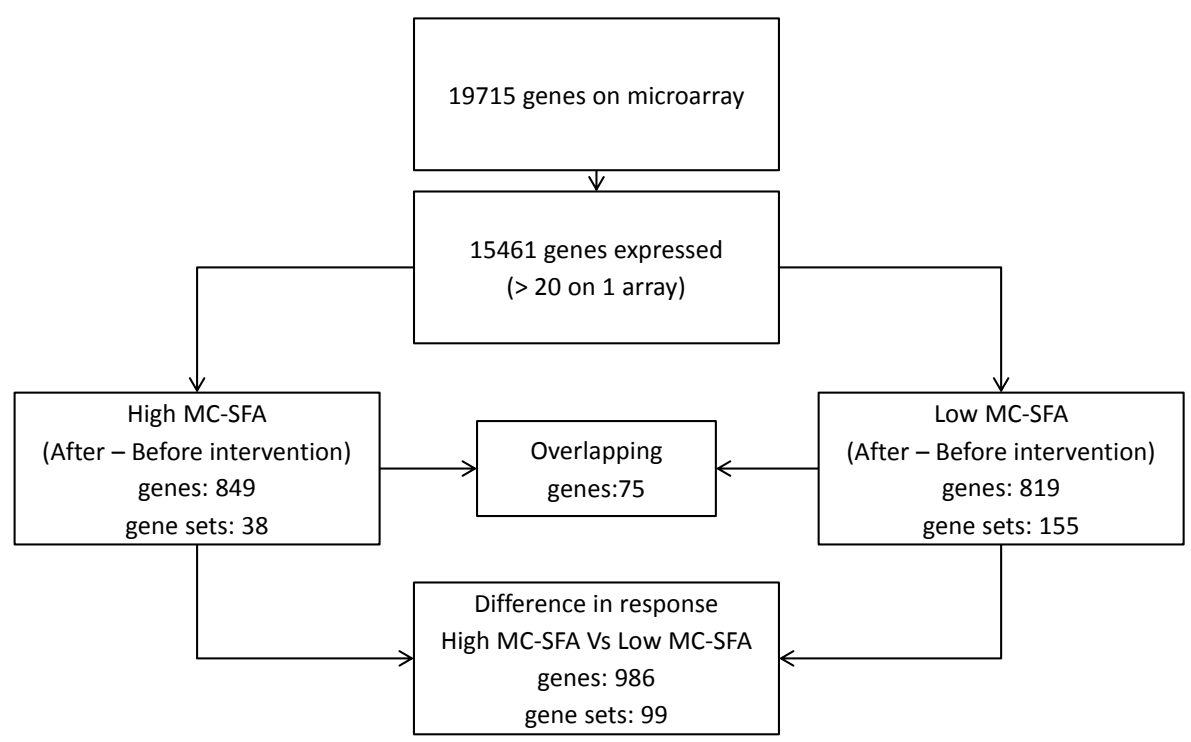

Figure 4-2. Flow chart of gene selection methods and number of significantly differentially expressed genes.

Genes were considered significantly different if $\mathrm{P}<0.05$. 

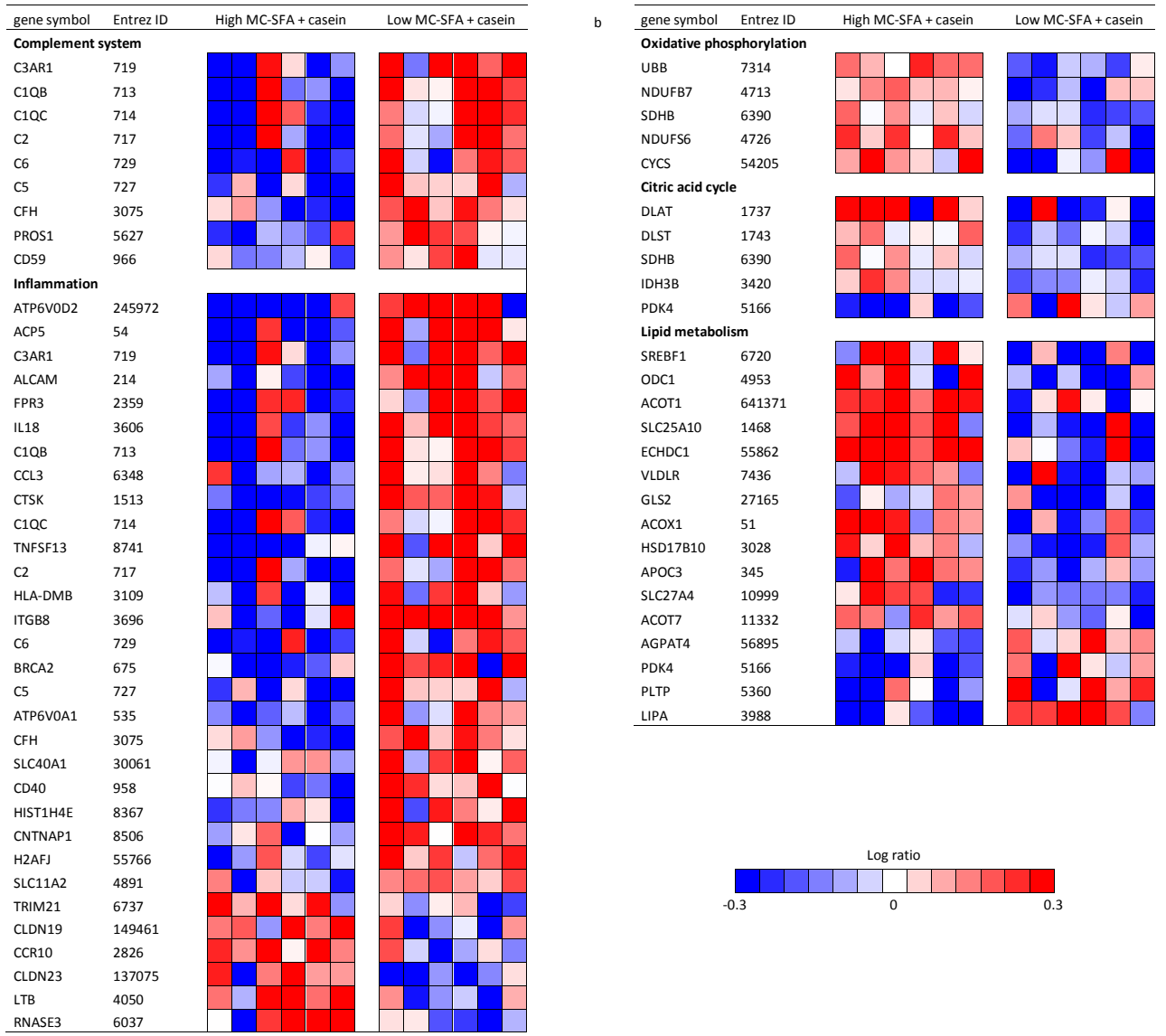

Figure 4-3. Heatmap showing log ratios per individual of the differentially expressed genes in gene sets related to a) complement system and inflammation, and b) oxidative phosphorylation, citric acid cycle and lipid metabolism. 

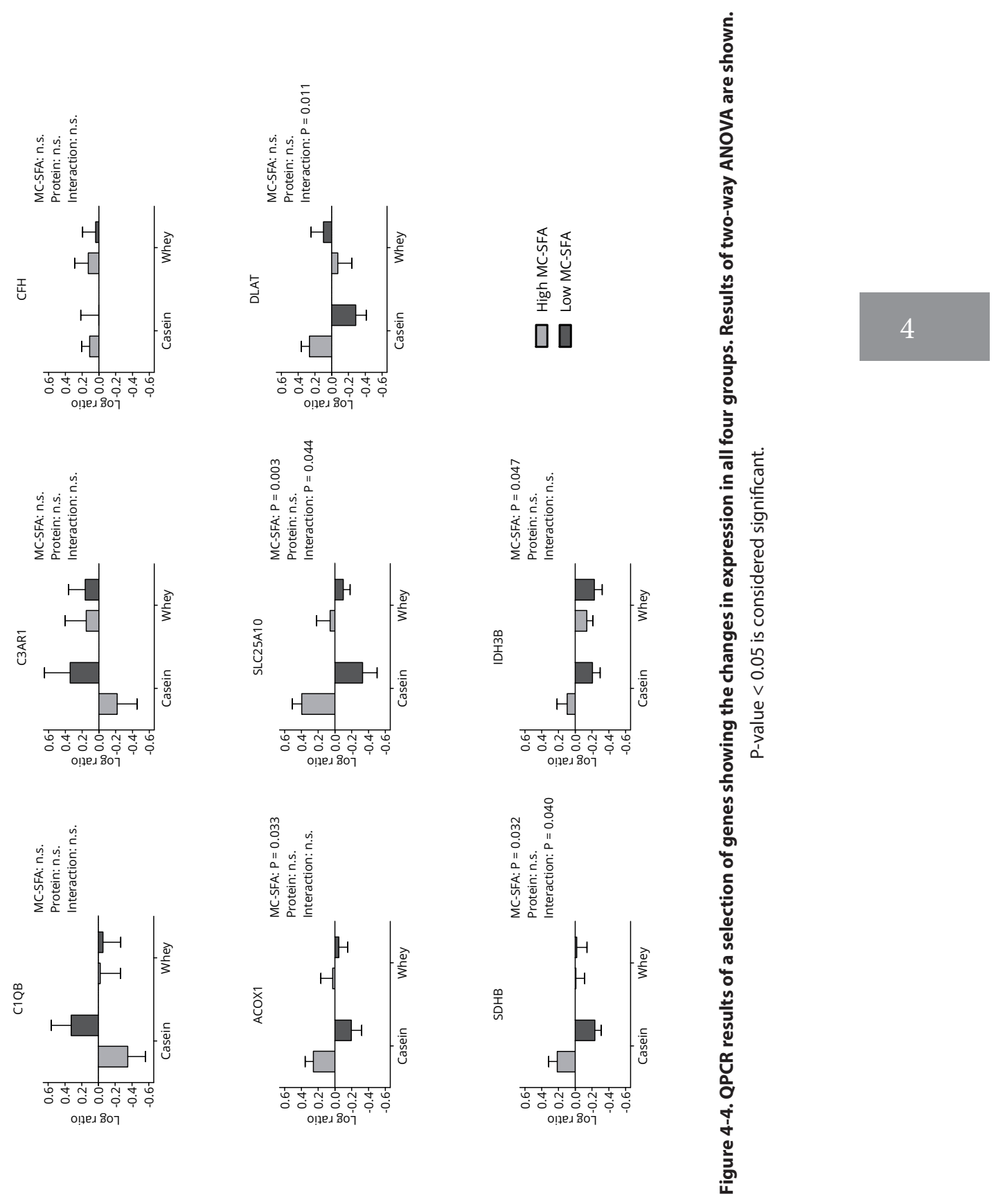


\section{Supplemental Tables}

Supplementary Table S4-1. Primer sequences used to quantify gene expression by qPCR.

\begin{tabular}{lll}
\hline Gene & Forward primer & Reverse Primer \\
\hline ACTB & GAAGAGCTACGAGCTGCCTGA & GAACCGCTCATTGCCAATG \\
PPIA & TCTTTGGGACCTTGTCTGCAA & CCCACCGTGTTCTTCGACAT \\
C1QB & AGGTGAATCGGGAGACTACAA & CACTGCGGGGCTCATAATTG \\
C3AR1 & CCCTACGGCAGGTTCCTATG & GACAGCGATCCAGGCTAATGG \\
CFH & GTGAAGTGTTACCAGTGACAGC & AACCGTACTGCTTGTCCAAAA \\
ACOX1 & ACTCGCAGCCAGCGTTATG & AGGGTCAGCGATGCCAAAC \\
SLC25A10 & ACCTGCTCAAGGTGCATCTG & CAGGGAGTAGGTCATCTGTCTG \\
DLAT & CGGAACTCCACGAGTGACC & CCCCGCCATACCCTGTAGT \\
SDHB & ACAGCTCCCCGTATCAAGAAA & GCATGATCTTCGGAAGGTCAA \\
IDH3B & TAGTGCAGAATACGCAGTCTTTG & CAGCATGTTGGAAGCCGAC \\
\hline
\end{tabular}

Supplementary Table S4-2. Baseline characteristics of the 12 participants in the high MC-SFA + casein and the low MC-SFA + casein groups in which subcutaneous adipose tissue gene expression microarrays were performed. $^{a}$

\begin{tabular}{llll}
\hline & High MC-SFA + casein (N=6) & Low MC-SFA + casein (N=6) & P-value \\
\hline Male/female ratio & $4 / 2$ & $4 / 2$ & 0.91 \\
Age (years) & $63 \pm 6$ & $64 \pm 16$ & 0.69 \\
Total cholesterol (mmol/L) & $6.3 \pm 1.1$ & $5.1 \pm 1$ & $<0.05$ \\
LDL cholesterol (mmol/L) & $4.1 \pm 0.9$ & $3 \pm 0.8$ & 0.83 \\
HDL cholesterol (mmol/L) & $1.5 \pm 0.4$ & $1.6 \pm 0.4$ & 0.22 \\
Triglycerides (mmol/L) & $1.6 \pm 1$ & $1 \pm 0.4$ & 0.96 \\
Waist circumference (cm) & $104.2 \pm 9.8$ & $103.9 \pm 9.3$ & 0.95 \\
Hip circumference (cm) & $106.9 \pm 3.7$ & $107.2 \pm 7.6$ & 0.97 \\
Waist to hip ratio & $0.97 \pm 0.06$ & $0.97 \pm 0.08$ & 0.96 \\
Weight (kg) & $85.5 \pm 15.2$ & $85.1 \pm 11.7$ & 0.31 \\
Height (cm) & $1.78 \pm 6.84$ & $1.74 \pm 6.08$ & 0.68 \\
BMI (kg/m2) & $27 \pm 4.9$ & $28 \pm 3.3$ & 0.20 \\
Systolic blood pressure (mmHg) & $126 \pm 14$ & $136 \pm 13$ & 0.39 \\
Diastolic blood pressure (mmHg) & $76 \pm 7$ & $80 \pm 8$ & \\
\hline
\end{tabular}

${ }^{a}$ Data presented as mean $\pm \mathrm{sd}$. Means were compared using independent t-tests and corresponding P-values are shown. 


\section{Supplemental Figures}

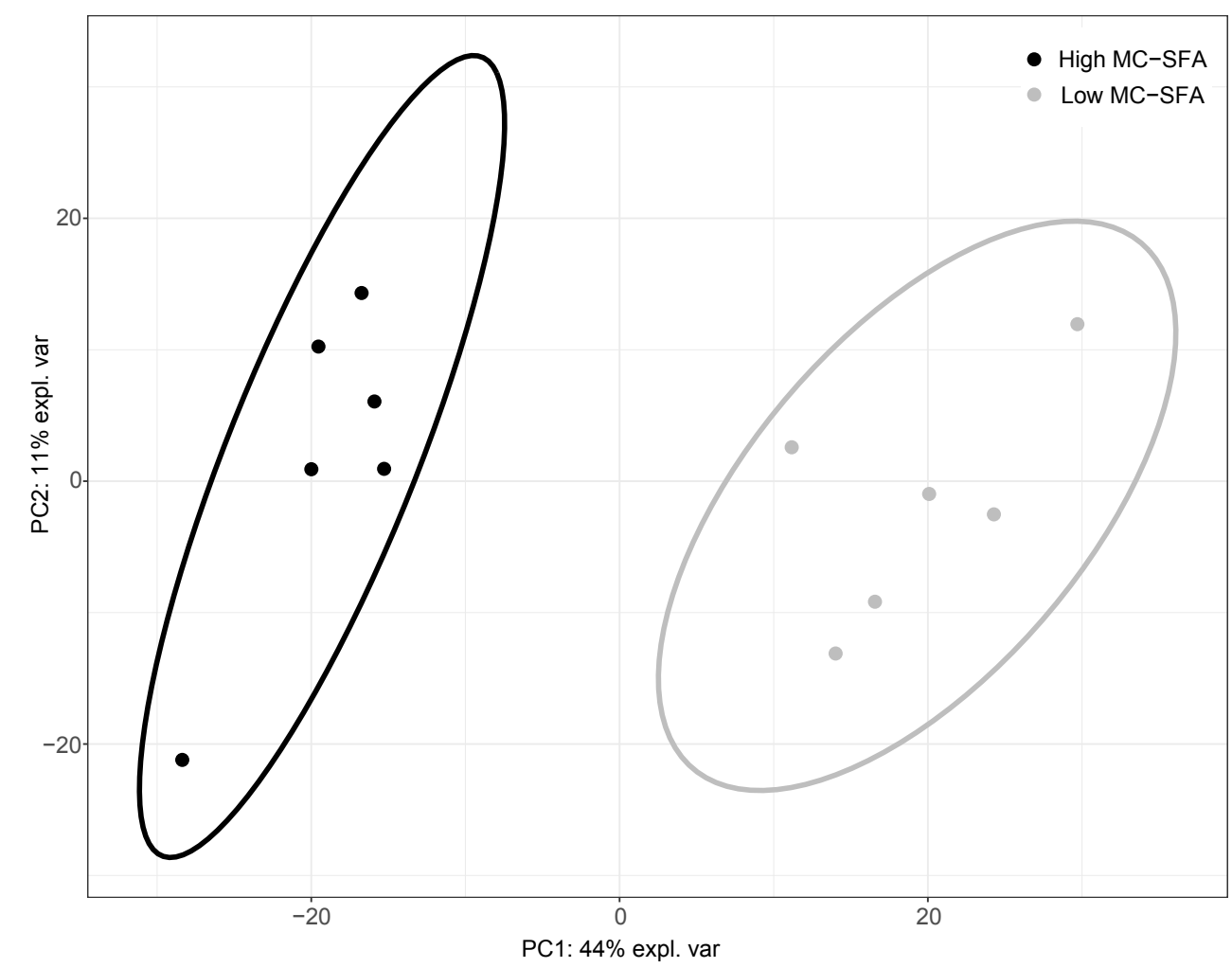

Supplementary Figure S4-1. Principal component analysis of changes (log2-ratios) in expression of genes differentially regulated between the high MC-SFA + casein (black dots) and low MC-SFA + casein group (grey dots).

Every dot represent one subject. 


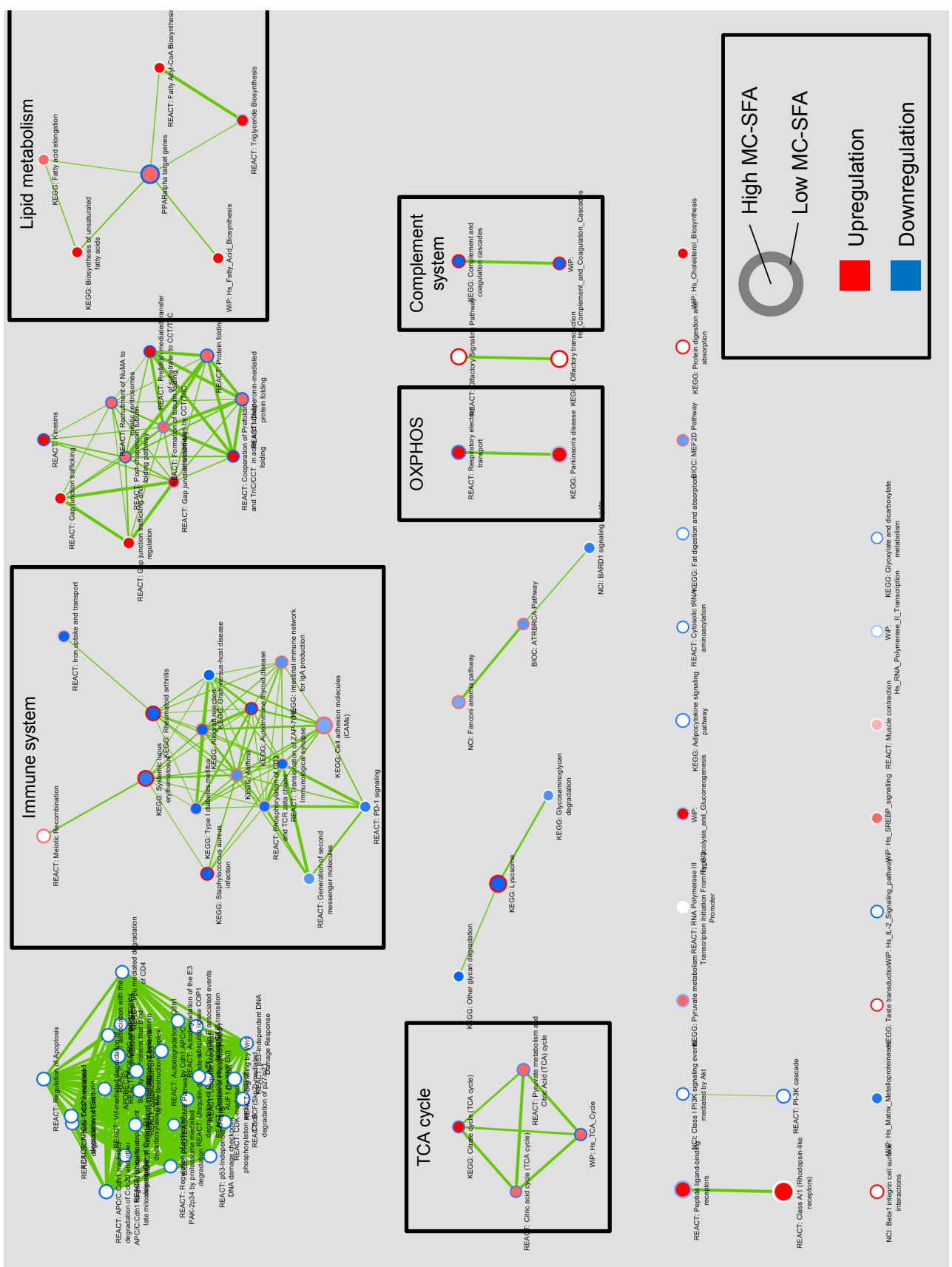


Supplementary Figure S4-2. Enrichment map showing gene sets significantly differentially changed in the high MC-SFA + casein compared to the low MC-SFA + casein group.

Nodes represent gene sets, whereas the size of the node reflects the number of genes in the gene set. Dotted lines indicate overlapping genes between gene sets. Node color indicates up-regulation (red), down-regulation (blue) or not affected (white) in the high MC-SFA + casein group (inside node) or the low MC-SFA + casein (outer node) subjects. Clusters of related gene sets were assigned a label based on the gene sets present in the cluster. Abbreviations: BIOC, BioCarta Pathway Diagrams; KEGG, Kyoto Encyclopedia of Genes and Genomes database; $\mathrm{NCl}$, Nature Pathway Interaction database; REACT, Reactome knowledgebase; WIP_HS, WikiPathways Homo Sapiens. 


\section{Added value of using high-fat challenges to determine a high-fat high-calorie diet-induced shift towards the metabolic syndrome as measured in blood cell transcriptome}

Juri Matualatupauw, Lydia Afman and Jildau Bouwman 


\section{Abstract}

\section{Background}

Differences in white blood cell whole genome gene expression responses to a high-fat challenge have been observed between lean and obese individuals. It is unknown if a high fat challenge combined with transcriptomics is able to distinguish smaller changes in health status, such as those induced by a short-term high-calorie diet. We aimed to identify the additional value of determining whole genome gene expression changes in response to a high-fat challenge compared to assessment at fasting only upon a 4-week high-fat high-calorie diet. In addition, we aimed to identify whether this diet can induce a shift in gene expression profiles in healthy subjects towards metabolic syndrome-like gene expression profiles.

\section{Methods}

Healthy subjects underwent a 4-week high-fat high-calorie diet. High-fat challenges were performed in these subjects before and after the diet and in subjects with the metabolic syndrome. Whole blood gene expression was measured at fasting and at 120 and $360 \mathrm{~min}$ after the high-fat challenges.

\section{Results}

After the high-fat high-calorie diet, the number of probes and pathways changed was $\sim 2$ and $\sim 3$ times higher, respectively, at fasting compared to post-prandial after the high fat challenge. Moreover, upon the high-fat high-calorie diet, a shift in gene expression profiles towards the metabolic syndrome was observed in 10 pathways at fasting, whereas after the high fat challenge this shift towards the metabolic syndrome was observed in one pathway at $120 \mathrm{~min}$ and one pathway at $360 \mathrm{~min}$.

\section{Discussion}

Compared to gene expression responses upon a high fat challenge, fasting gene expression profiles are more responsive to a 4-week high-fat high-calorie diet and show a larger shift towards the metabolic syndrome. 


\section{Introduction}

One of the main challenges in nutritional science is the measurement of diet-induced health effects. As the effects of nutrition are relatively small and may take decades to become apparent, researchers are focusing on developing more sensitive measurements of health status than conventional static measurements at fasting. A new approach to detect early and small disturbances in metabolic health is to examine the ability of a person to adapt to a metabolic challenge, which is known as phenotypic flexibility (1). Metabolic challenge tests are increasingly being used in nutritional research and are hypothesized to enlarge the relatively small effects of nutrition on health. Several types of challenge tests are used, such as the oral glucose tolerance test, the mixed meal test and the high-fat test. In the latter, subjects consume a large amount of fat, usually as a shake, and measurements are performed in the fasted state and at several time points post-prandially over the course of several hours.

Combining these high-fat challenge tests with high-throughput -omics tools, such as transcriptomics, has proven valuable for magnifying differences in health status. Esser et al. (2) showed that differences in white blood cell gene expression profiles between lean and obese individuals were larger after a high-fat challenge compared to the fasted state. In addition to this use as a biomarker, blood cell transcriptome analyses have also provided us with an increased mechanistic understanding of the changes in the cellular processes caused by diet that may be associated with metabolic disease (2-4).

To examine whether challenge tests can also be used to study more subtle changes in metabolic health caused by diet, we recently published an intervention study, in which the response of plasma metabolites to a high-fat challenge was examined in healthy subjects both before as well as after a 4-week high-fat high-calorie diet and compared to responses in MetS subjects (5). Several plasma markers, shifted towards the levels of the MetS subjects after the diet. For example, the increases in postprandial levels of some eicosanoids (11(S)-HETE, 12(S)-HETE, and 19,20-DiHoPe) were significantly diminished in healthy subjects by the high-fat highcalorie diet, resulting in a response similar to that of MetS subjects.

As white blood cell gene expression changes in response to a high-fat challenge have been shown to be different between lean and obese individuals, it is possible that these may also be used to assess more subtle differences in health status, such as those induced by a shortterm high-calorie diet. Therefore, in this study we will answer the following questions. Firstly, we will examine the additional value of studying whole genome gene expression changes in 
response to a high-fat challenge compared to studying only fasting gene expression profiles. Secondly, we examine whether the 4 week high-fat high-calorie diet can induce a shift in gene expression profiles in the healthy subjects towards more MetS-like profiles, both at fasting and post-prandially.

\section{Methods}

\section{Subjects}

Men aged between 35 and 65 years were recruited from a pool of volunteers from the Netherlands Organisation for Applied Scientific Research (TNO) database and with the assistance of a recruitment agency. In total, 20 men participated in the study. Of these men, 10 were healthy, and 10 had two or more characteristics of MetS. Inclusion criteria for the healthy men were age 35-65 yr, BM 23-30 kg/m2, normal Dutch eating habits and eating without restriction, defined as a score of $<3.25$ on the Dutch Restrained Eating Questionnaire. Inclusion criteria for the MetS group were age 35-65 yr, BMI 23-40 kg/m2, normal Dutch eating habits, and 2 or more cardiometabolic risk factors, defined as waist circumference $\geq$ $94 \mathrm{~cm}$, TGs $\geq 1.7 \mathrm{mM}$, HDL cholesterol $<0.9 \mathrm{mM}$, systolic blood pressure $\geq 130 \mathrm{~mm} \mathrm{Hg}$ and diastolic blood pressure $\geq 85 \mathrm{~mm} \mathrm{Hg}$ and fasting glucose $\geq 5.6 \mathrm{mM}$. Exclusion criteria for both groups were a history of medical or surgical events that may significantly affect the study outcome, including medication for diabetes, cholesterol-lowering medication, eating disorders, food allergy, smoking, consuming more than $28 \mathrm{U} / \mathrm{wk}$ of alcohol, and exercising more than $3 \mathrm{~h} / \mathrm{wk}$. Written informed consent was obtained from each participant after receiving an explanation of the procedures. Before the start of the study, all subjects underwent a screening that involved an anamnesis about medical history, lifestyle and eating behavior, measurement of body composition, blood pressure, waist circumference, and collection of blood for clinical laboratory tests.

\section{Study design}

The design of the study was described in detail previously (5) and is visualized in Figure 5-1. Briefly, 9 healthy and 10 MetS men were included in this study. It should be noted that compared to the earlier paper, we have one less healthy subject and one more MetS subject. The healthy subjects underwent a 4 week high-fat high-calorie dietary intervention. The intervention diet consisted of a fixed amount of commercially available foods high in fat, 
sugar, or both and was consumed on top of their habitual diet (see below for details). Before and after the 4 week intervention period, the healthy subjects underwent a high-fat challenge consisting of whipping cream, water and sugar (see below for details). The MetS subjects underwent the high-fat challenge once. An independent medical ethics committee (Medical Ethics Committee (METC) of Brabant, Tilburg, The Netherlands) approved the research protocol.

\section{Intervention diet}

Once a week, the healthy subjects received a shopping bag with the items of the high-fat high-calorie diet. They were asked to maintain their habitual diet and lifestyle. In addition to their habitual diet, they were asked to consume the items of the high-fat high-calorie diet. The calculated nutritional composition of the weekly diet was $36,328 \mathrm{~kJ}, 215 \mathrm{~g}$ protein, $440 \mathrm{~g}$ fat, and $965 \mathrm{~g}$ carbohydrates, resulting in a daily surplus of 5,190 kJ (= $1240 \mathrm{kcal}$ ), $31 \mathrm{~g}$ protein, 63 $\mathrm{g}$ fat, and $138 \mathrm{~g}$ carbohydrates. Subjects were instructed to divide their dietary items equally over the course of the week. For the final week, they received an additional snack pack on top of the intervention diet, containing 12,805 kJ, $72 \mathrm{~g}$ protein, $179 \mathrm{~g}$ fat and $290 \mathrm{~g}$ carbohydrates, in order to maintain the increase in body weight.

\section{High-fat shake}

The high-fat challenge was a shake consisting of $53 \%$ whipped cream, $3 \%$ sugar, and $44 \%$ water. The volume of the shake was $500 \mathrm{ml}$. The nutritional composition of the shake per $100 \mathrm{~g}$ was $640 \mathrm{~kJ}, 1.6 \mathrm{~g}$ protein, $16 \mathrm{~g}$ fat, and $3.2 \mathrm{~g}$ carbohydrates. The subjects were instructed to restrict their physical activity and alcohol consumption the day before test days. A standardized lowfat meal (1922 kJ, $30 \mathrm{~g}$ protein, $7 \mathrm{~g}$ fat, and $64 \mathrm{~g}$ carbohydrates) was provided for consumption at home the evening before the test days. A fasting blood sample $(\mathrm{t}=0 \mathrm{~min})$ and two postprandial blood samples $(t=120 \mathrm{~min}$ and $\mathrm{t}=360 \mathrm{~min})$ were used for the microarray analysis.

\section{RNA isolation and microarray analysis}

Blood samples were taken before consumption of the shake in the fasted state $(t=0 \mathrm{~min})$ and at two postprandial time points $(\mathrm{t}=120 \mathrm{~min}$ and $\mathrm{t}=360 \mathrm{~min})$. At these time points, 2.5 $\mathrm{ml}$ of blood was collected in PAXgene ${ }^{\oplus}$ Blood RNA Tubes. RNA extraction was performed as described previously in detail (6). Briefly, total RNA was extracted using glass beads and RNAzol (Campro Scientific, Veenendaal, The Netherlands). RNA integrity was examined using the RNA 6000 Nano Lab-on-a-Chip kit and a bioanalyzer 2100 (Agilent Technologies, 
Amstelveen, The Netherlands). The Illumina ${ }^{\circledR}$ TotalPrep $^{\text {Tm }}$ RNA Amplification Kit (Ambion, art. No.AM-IL1791) was used to synthesize biotin labeled cRNA starting with 500 ng total RNA. The concentration of the labeled cRNA was measured using the Nanodrop spectrophotometer. The amount of biotinylated cRNA which was hybridized onto the Illumina HumanHt12-v4 Expression Beadchip was 750 ng. Illumina's Genomestudio v1.1.1 software with the default settings advised by Illumina was used for Gene Expression analysis.

\section{Microarray analysis}

Microarray QC and pre-processing were performed using Arrayanalysis.org (7). Three samples did not pass QC-criteria and were left out of further analysis. Probe expression values were calculated using the neqc algorithm from the limma package (8), which performs background correction, quantile normalization and $\log 2$-transformation of the data. We filtered out low expressing probes by selecting only the probes with a detection p-value < 0.01 in at least 5 arrays for further analysis. Further statistical analysis on the remaining 17,184 probes was performed in $\mathrm{R}$ using the limma package. For all comparisons, probes were defined as significantly different when the P-value was $<0.05$. Differences in baseline expression between the healthy and MetS subjects were identified using the contrast (MetS_ t0- Healthy_before_t 0 ). Expression changes in response to the challenge at $t=120 \mathrm{~min}$ in the healthy subjects after intervention were identified using the contrast (Healthy_after_t120Healthy_after_t 0 ). Differences in challenge response at $\mathrm{t}=120 \mathrm{~min}$ between the MetS and the healthy subjects were identified using the contrast ((MetS_t120-MetS_t0)-(Healthy_before_ t120-Healthy_ before_t0)). For the differences at other time points and between different groups, contrasts were defined in similar fashion. To take into account the paired design of our study, subjects were treated as random effects using the "duplicateCorrelation" function.

\section{Pathway analysis}

To examine changes in gene expression pathways, we performed gene set enrichment analysis (GSEA; http:/www.broad.mit.edu/gsea) (9). Genes were ranked based on the t-statistic and analyzed for over- or underrepresentation in predefined gene set using pre-ranked GSEA. In order to end up with one value for each gene, we used the highest absolute t-statistic value when multiple probes were annotated to the same gene. The gene set database was custommade and consisted of gene sets from the Biocarta, KEGG, Reactome and Wikipathways databases. We used a cut-off of FDR q $<0.25$ for statistical significance. Significant gene sets were visualized in Cytoscape using enrichment map (10). 


\section{Statistics}

Differences in subject characteristics between the two groups were analyzed using independent t-tests Changes in subject characteristics in the healthy group were analyzed using paired t-tests. P-values $<0.05$ were considered significant. IBM SPSS Statistics, version 22.0.0.1 was used for all analyses.

\section{Results}

\section{Subject characteristics}

Several metabolic markers have been analyzed and discussed in detail previously (5). In this study, microarray data was available in 9 healthy subjects and 10 MetS subjects. Characteristics of these subjects are shown in Table 5-1 and have been published before for a similar subset of the population (5). The MetS subjects had a higher body weight, BMI, total cholesterol, LDL cholesterol, glucose, insulin, and waist-to-hip ratio compared to the healthy subjects. After the high-fat high-calorie diet, the healthy subjects showed an increase in body weight, BMI, triglycerides, insulin and waist-to-hip ratio (Table 5-1).

\section{Microarray analysis}

High-fat challenges were performed in subjects with MetS and in healthy subjects before and after the dietary intervention. During each of these challenges, we performed microarray analyses at fasting and at 120 and 360 min post-prandially. To identify if high-fat challenges provide additional information compared to fasting measurements we examined the changes in gene expression both at fasting as well as during the high-fat challenge. To determine if the 4-week high-fat high-calorie diet induced a shift in gene expression towards a more MetS-like profile, we examined the overlap between subjects with MetS vs healthy subjects, and healthy subjects after vs before invention, both at fasting and post-prandially. All these analyses were done at probe (Figure 5-2) and pathway level (Table 5-2).

\section{Do high-fat challenges provide additional information compared to fasting?}

We examined whether measurement of gene expression responses upon a high-fat challenge could provide additional information compared to measurement of gene expression profiles at fasting.. Firstly, we examined how many probes were differently changed by the intervention diet in the healthy subjects. At fasting, these were 2007 probes (Figure 5-2a, dashed lines), 
whereas in the response to the high-fat challenge this number was lower, with 1170 and 716 probes differentially expressed at 120 and 360 min respectively after the challenge upon the 4 week intervention (Figure 5-2b, dashed lines).

We also examined the above research question on the pathway level. After the intervention diet, at fasting healthy subjects showed changes in expression of 568 gene sets, of which 71 were upregulated and 497 were downregulated. The post-prandial response to the challenge showed changes in 3 gene sets ( 3 upregulated, 0 downregulated) at $120 \mathrm{~min}$ and 25 gene sets (1 upregulated, 24 downregulated) at $360 \mathrm{~min}$. The gene sets that were changed and the direction of changes induced by the intervention at fasting and post-prandially are summarized in Table 5-2.

\section{Does the intervention diet cause a shift towards the metabolic syndrome?}

We examined whether the intervention diet induced a shift in gene expression profiles in the healthy subjects upon the 4 weeks high fat high calorie intervention towards the gene expression profiles of the MetS subjects. This was done both at fasting and for the high-fat challenge response at the probe and pathway level.

We determined the overlap of significantly differentially expressed probes due to the diet in healthy subjects and the probes that were significantly different between MetS and healthy subjects before the intervention. At fasting this overlap was found to be 319 probes (Figure 5-2a, dotted lines), and in response to the high-fat challenge the overlap was in a similar range with 287 and 192 probes at 120 and 360 min respectively (Figure 5-2b, dotted lines).

As can be observed from the highlighted pathways in Table 5-2, at fasting, a large overlap is present between the changes induced by the diet in healthy subjects and the pathways differently expressed in the MetS subjects. Pathways related to oxidative phosphorylation, and translation showed an upregulation upon the high-fat high-calorie diet and a shift in expression towards MetS. Pathways related to antigen presentation, B cell receptor signaling, chromatin organization, insulin receptor signaling, lipid metabolism, notch signaling, $\mathrm{T}$ cell receptor signaling and toll-like receptor cascades showed a downregulation upon the high-fat high-calorie diet and a shift in expression towards MetS.

Postprandially, two pathways (Table 5-2, highlighted in yellow) were changed by the diet in healthy subjects that were also different between MetS and healthy. Healthy subjects before intervention showed a downregulation of the chaperone gene activation pathways at $120 \mathrm{~min}$. After intervention, this down regulation was no longer present, resembling the post-prandial 
response of MetS subjects. To illustrate how the expression of the individual genes in the chaperone activation pathways were changed post-prandially a heatmap with individual gene expression changes of al subject at 120 min postprandial is shown in Figure 5-3. At $360 \mathrm{~min}$ after the challenge, expression of genes in the TLR cascades pathways showed a shift towards MetS. In the healthy subjects before intervention, this pathway was upregulated, whereas after intervention, this upregulation was less pronounced, which was similar to the MetS subject, that showed no postprandial change in TLR cascade pathways. To illustrate how the expression of the individual genes in the TLR cascade pathways were changing postprandially a heatmap with individual gene expression changes of all subjects at $360 \mathrm{~min}$ postprandial is shown in Figure 5-4.

\section{Discussion}

The combination of high-fat challenge tests with transcriptome measurements has been suggested as a suitable tool for picking up subtle changes in health status. In this study, we used a 4-week high-fat high-calorie diet to induce a small change in health status in apparently healthy subjects. After this dietary intervention, the number of probes and pathways changed was $\sim 2$ and $\sim 3$ times higher at fasting compared to post-prandially. Moreover, after the highfat high-calorie diet, at fasting, a shift towards MetS was observed in 10 pathways, whereas postprandial gene expression responses showed this shift towards MetS in one pathway at 120 min and one pathway at $360 \mathrm{~min}$.

In this study, first, we examined the additional value of studying whole genome gene expression changes in response to a high-fat challenge compared to studying fasting gene expression profiles only. The $\sim 2$ times higher number of probes changed and the many more pathways changed at fasting compared to post-prandially shows that fasting changes are much more pronounced than post-prandial high-fat challenge-induced changes after the 4 week high-fat high-calorie diet. This indicates that the high-fat high-calorie diet-induced changes in fasting gene expression likely provide more information than using high-fat challenges. A potential explanation may be the loss of power as the fasting changes are based on a comparison of two timepoints, before and after intervention, whereas the post-prandial measurements are based on six timepoints, fasting and postprandial, both before and after intervention. The additional variation that is induced by including these extra timepoints may decrease statistical power. Another likely explanation may the combination of the high-fat high-calorie intervention 
with the high fat challenges, as the daily exposure to high-fat meals from the intervention may have caused changes in the response to high-fat meals, such as the high-fat challenge. To sum up, based on this study, the additional value of high-fat challenge tests to study the effects of a high-fat high-calorie dietary intervention remains questionable.

We also examined whether the 4-week high-fat high-calorie diet induced a shift in gene expression profiles towards the profiles of MetS subjects. At fasting, in healthy subjects, the 4 week diet intervention caused changes in gene expression that were similar to the differences in gene expression observed between MetS subjects and healthy subjects. These findings coincide with the diet-induced changes in body weight, BMI, triglycerides, insulin and waistto-hip ratio, which were all going in the direction of the MetS subjects. The gene expression response to the challenge remained largely unchanged in healthy subjects after intervention diet, with expression of less genes and pathways changed when compared to diet-induced changes at fasting.

At fasting, we observed a higher expression of OXPHOS genes in MetS subjects compared to healthy subjects before the intervention and an increase in expression in healthy subjects after the diet intervention. Other studies have also reported increased OXPHOS gene expression in whole blood in in obese vs lean subjects (11) and MetS vs healthy subjects (12). While the cause of this increase in expression is not completely understood, it has been suggested that increased OXPHOS gene expression may be caused by an increased energy demand with obesity (11).

Other pathways that showed a change at fasting due to the intervention in the direction of MetS were related to inflammation, such as T-cell receptor signaling, B-cell receptor signaling and TLR cascades. Genes in these pathways were lower expressed in MetS subjects compared to healthy subjects and showed a decrease in expression in healthy subjects after the dietary intervention. Since MetS is associated with a low-grade chronic inflammation $(13,14)$, we did not expect this lower expression in Mets subjects and the downregulation in gene expression upon the intervention. One might expected that a constant exposure in blood to slightly elevated pro-inflammatory molecules such as TNF- $\alpha$ and IL-6 (15) results in increased expression of inflammation-related genes in blood cells. Other studies did indeed show an increased expression of inflammation-related genes in subjects with MetS compared to healthy controls (16). However, one study examined the effects of a 7-day high-fat diet in healthy men and observed a decrease in TLR2 protein expression in PBMCs and a reduced ex-vivo cytokine excretion upon stimulation with a TLR2 agonist (17). These findings are 
consistent with our results, though the mechanism behind the diminishing effects of a highfat diet on TLR cascade pathways remains unclear.

Genes related genes related to chaperone gene activation showed a switch in challenge response at 120 min towards MetS induced by the diet. After the diet, the downregulation of these pathways was no longer present, which was also observed in the MetS subjects. Chaperone gene activation is part of the unfolded protein response (UPR), which is a cellular stress response that is activated in situations of endoplasmatic reticulum stress (18). One might therefore expect an activation instead of a downregulation, which was also observed in other studies where an increased expression of ER stress-regulated genes was observed in blood cells of subjects with the MetS compared to healthy controls after an oral glucose tolerance test (19). Furthermore, the unfolded protein response has been shown to be activated during obesity in other tissues, such as hypothalamus, liver, fat, muscle and pancreatic $\beta$-cells in studies in humans and rodents (20). It is unclear why our findings are discrepant with these observations. However, much of the triggering mechanism and physiological roles of the UPR in relation to the MetS is not completely understood $(20,21)$.

Another pathway that showed a change in challenge response after the high-fat high-calorie in healthy subjects was the TLR cascade pathway. The upregulation of this pathway at 360 min was diminished after the 4 week high-fat high-calorie diet, resulting in a more MetS-like response, as MetS subjects did not show a significant change in expression of genes involved in the TLR cascade pathways. Interestingly, as discussed previously, at fasting, we observed a lower expression of these pathways in MetS subjects and a downregulation in healthy subjects upon the diet compared to the healthy subjects before the diet. Several studies have shown that saturated fatty acids are able to active TLR- cascade pathways (22, 23), leading to NFkB-activation and subsequent increase in gene expression of pro-inflammatory genes (23). Despite the fact that pro-inflammatory gene expression was already lower at fasting in subjects with the MetS, they do not show an increase in pro-inflammatory gene expression in response to a high-fat load, which may indicate that, potentially, they have a reduced cellular adaptation capacity. In this regard, the 4-week high-fat high-calorie diet may have induced changes in healthy subjects towards a MetS-like state of reduced cellular adaptation capacity. A major strength of this study was the use of a high-fat high-calorie diet that induced weight gain and, likely, a change in metabolic health status. Most studies take the opposite approach and aim to increase metabolic health, either by caloric restriction and resultant weight reduction or by using health-promoting diets. A limitation of the current study is that we did 
not include a placebo group and, therefore, we cannot confirm that all of the changes observed after the diet intervention were specifically caused by the diet. Another limitation of this study is the relatively small size of the groups of 9 and 10 subjects. Therefore, to minimize the chance of false-positive results, we focused our analyses mainly on the pathway level. We did observe distinct differences in the gene expression response to the high-fat challenge between healthy and MetS subjects, indicating that the differences between these groups may be quite large. Conversely, changes in high-fat challenge response induced by the diet in healthy subjects were quite small despite the fact that these were within subject changes. It should be noted that the diet was only 4 weeks long and led to changes in subject characteristics that were not yet at the levels of MetS subjects. Furthermore, it is possible that the daily exposure to a high-fat high-calorie diet may have affected the response to a high fat meal by a potential habituation to the high-fat meals that were part of the diet. Possibly, dietary interventions with a different dietary composition may be able induce larger changes in high-fat challenge response.

In conclusion, fasting whole blood whole genome gene expression profiles are highly responsive to a 4-week high-fat high-calorie diet, showing changes in many pathways in the direction of a MetS-like gene expression profile. High-fat challenge responses in healthy subjects show only small changes upon the dietary intervention and a minimal shift in the direction of the metabolic syndrome. This indicates that fasting gene expression profiles are more responsive to the effects of a 4-week high-fat high-calorie diet than high-fat challengeinduced gene expression responses.

\section{References}

1. Stroeve JH, van Wietmarschen H, Kremer BH, van Ommen B, Wopereis S. Phenotypic flexibility as a measure of health: the optimal nutritional stress response test. Genes \& nutrition. 2015;10(3):459.

2. Esser D, van Dijk SJ, Oosterink E, Lopez S, Muller M, Afman LA. High fat challenges with different fatty acids affect distinct atherogenic gene expression pathways in immune cells from lean and obese subjects. Mol Nutr Food Res. 2015;59(8):1563-72.

3. Bouwens M, Grootte Bromhaar M, Jansen J, Muller M, Afman LA. Postprandial dietary lipid-specific effects on human peripheral blood mononuclear cell gene expression profiles. The American journal of clinical nutrition. 2010;91(1):208-17.

4. Matone A, O'Grada CM, Dillon ET, Morris C, Ryan MF, Walsh M, et al. Body mass index mediates inflammatory response to acute dietary challenges. Mol Nutr Food Res. 2015;59(11):2279-92.

5. Kardinaal AF, van Erk MJ, Dutman AE, Stroeve JH, van de Steeg E, Bijlsma S, et al. Quantifying phenotypic flexibility as the response to a high-fat challenge test in different states of metabolic health. FASEB J. 2015;29(11):4600-13. 
6. Verschuren L, Radonjic M, Wielinga PY, Kelder T, Kooistra T, van Ommen B, et al. Systems biology analysis unravels the complementary action of combined rosuvastatin and ezetimibe therapy. Pharmacogenetics and genomics. 2012;22(12):837-45.

7. Eijssen LM, Goelela VS, Kelder T, Adriaens ME, Evelo CT, Radonjic M. A user-friendly workflow for analysis of Illumina gene expression bead array data available at the arrayanalysis.org portal. BMC genomics. 2015;16:482.

8. Ritchie ME, Phipson B, Wu D, Hu Y, Law CW, Shi W, et al. limma powers differential expression analyses for RNA-sequencing and microarray studies. Nucleic acids research. 2015;43(7):e47.

9. Subramanian A, Tamayo P, Mootha VK, Mukherjee S, Ebert BL, Gillette MA, et al. Gene set enrichment analysis: a knowledge-based approach for interpreting genome-wide expression profiles. Proceedings of the National Academy of Sciences of the United States of America. 2005;102(43):15545-50.

10. Merico D, Isserlin R, Stueker O, Emili A, Bader GD. Enrichment map: a network-based method for gene-set enrichment visualization and interpretation. PloS one. 2010;5(11):e13984.

11. Ghosh S, Dent R, Harper ME, Gorman SA, Stuart JS, McPherson R. Gene expression profiling in whole blood identifies distinct biological pathways associated with obesity. BMC medical genomics. 2010;3:56.

12. Tangen SE, Tsinajinnie D, Nunez M, Shaibi GQ, Mandarino LJ, Coletta DK. Whole blood gene expression profiles in insulin resistant Latinos with the metabolic syndrome. PloS one. 2013;8(12):e84002.

13. Eckel RH, Grundy SM, Zimmet PZ. The metabolic syndrome. Lancet. 2005;365(9468):1415-28.

14. Sutherland JP, McKinley B, Eckel RH. The metabolic syndrome and inflammation. Metabolic syndrome and related disorders. 2004;2(2):82-104.

15. Hotamisligil GS, Arner P, Caro JF, Atkinson RL, Spiegelman BM. Increased adipose tissue expression of tumor necrosis factor-alpha in human obesity and insulin resistance. The Journal of clinical investigation. 1995;95(5):2409-15.

16. Grayson BL, Wang L, Aune TM. Peripheral blood gene expression profiles in metabolic syndrome, coronary artery disease and type 2 diabetes. Genes and immunity. 2011;12(5):341-51.

17. Wan Z, Durrer C, Mah D, Simtchouk S, Little JP. One-week high-fat diet leads to reduced toll-like receptor 2 expression and function in young healthy men. Nutr Res. 2014;34(12):1045-51.

18. Boden G. Endoplasmic reticulum stress: another link between obesity and insulin resistance/inflammation? Diabetes. 2009;58(3):518-9.

19. Sage AT, Holtby-Ottenhof S, Shi Y, Damjanovic S, Sharma AM, Werstuck GH. Metabolic syndrome and acute hyperglycemia are associated with endoplasmic reticulum stress in human mononuclear cells. Obesity. 2012;20(4):748-55.

20. Cnop M, Foufelle F, Velloso LA. Endoplasmic reticulum stress, obesity and diabetes. Trends in molecular medicine. 2012;18(1):59-68.

21. Zhang K, Kaufman RJ. Signaling the unfolded protein response from the endoplasmic reticulum. The Journal of biological chemistry. 2004;279(25):25935-8.

22. Huang S, Rutkowsky JM, Snodgrass RG, Ono-Moore KD, Schneider DA, Newman JW, et al. Saturated fatty acids activate TLR-mediated proinflammatory signaling pathways. Journal of lipid research. 2012;53(9):200213.

23. Rocha DM, Caldas AP, Oliveira LL, Bressan J, Hermsdorff HH. Saturated fatty acids trigger TLR4-mediated inflammatory response. Atherosclerosis. 2016;244:211-5. 


\section{Tables}

Table 5-1. Subject characteristics of the healthy subjects before and after intervention and the MetS subjects.

\begin{tabular}{llll}
\hline & \multicolumn{2}{c}{ Healthy $(\mathbf{N}=9)$} & Metabolic syndrome \\
\cline { 2 - 4 } & Before intervention & After intervention & (N $=10)$ \\
\hline Body weight $(\mathrm{kg})$ & $78.2 \pm 8.4$ & $80.8 \pm 8.3^{*}$ & $95.9 \pm 8.3^{*}$ \\
BMI (kg/m2) & $24.0 \pm 1.9$ & $24.8 \pm 1.9^{*}$ & $29.0 \pm 2.1^{*}$ \\
Triglycerides (mmol/l) & $1.2 \pm 0.6$ & $2.0 \pm 0.8^{*}$ & $1.7 \pm 0.6$ \\
Cholesterol (total) (mmol/l) & $5.3 \pm 0.8$ & $5.7 \pm 0.9$ & $6.7 \pm 0.9^{*}$ \\
HDL cholesterol (mmol/l) & $1.3 \pm 0.3$ & $1.4 \pm 0.3$ & $1.3 \pm 0.2$ \\
LDL cholesterol (mmol/l) & $3.4 \pm 0.7$ & $3.4 \pm 0.8$ & $4.6 \pm 0.8^{*}$ \\
Waist-to-hip ratio & $0.89 \pm 0.04$ & $0.90 \pm 0.04^{*}$ & $0.95 \pm 0.04^{*}$ \\
Glucose & $5.1 \pm 0.3$ & $5.3 \pm 0.3$ & $6.0 \pm 0.3^{*}$ \\
Insulin & $4.5 \pm 1.6$ & $7.0 \pm 3.3^{*}$ & $14.0 \pm 4.6^{*}$ \\
\hline
\end{tabular}

*, significantly different from healthy subjects before intervention.

\section{Table 5-2. Differences in significantly expressed gene sets in each of the comparisons.}

GSEA results were visualized in enrichment maps and gene set clusters are summarized in this table. Shown are differentially expressed gene set clusters at fasting and post-prandially in response to the high-fat challenge at 120 and $360 \mathrm{~min}$. Arrows indicate direction of change ( $\uparrow$, upregulation; $\downarrow$ downregulation). For the post-prandial time points, only the pathways are shown that are significant in the comparison of healthy after intervention ( $t=4)$ vs before intervention $(t=0)$ or the comparison of MetS vs healthy before intervention $(t=0)$. Yellow highlighting indicate that these pathways are differentially expressed in both of these comparisons. 
High-fat challenges, shift towards metabolic syndrome

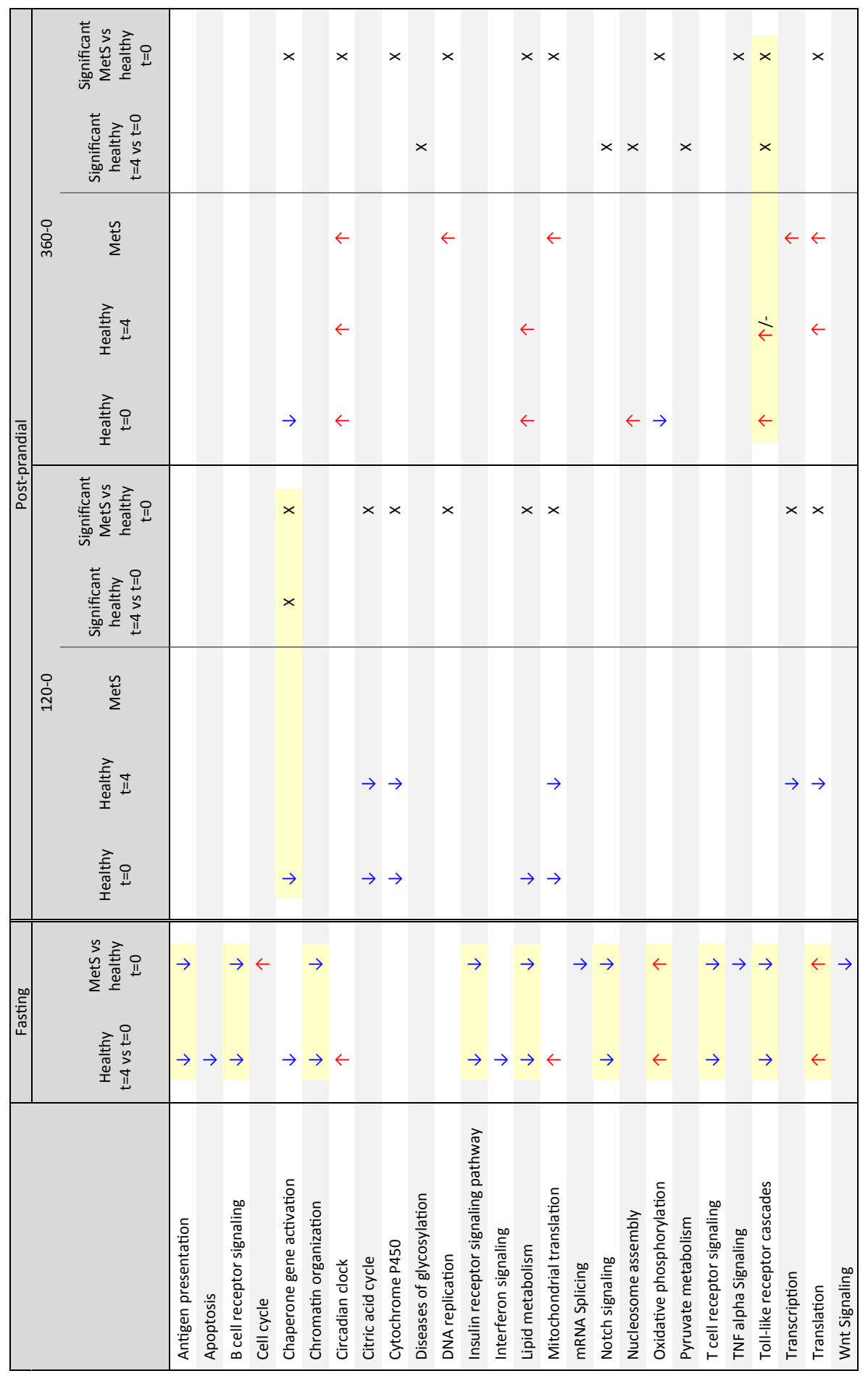




\section{Figures}

a Study design

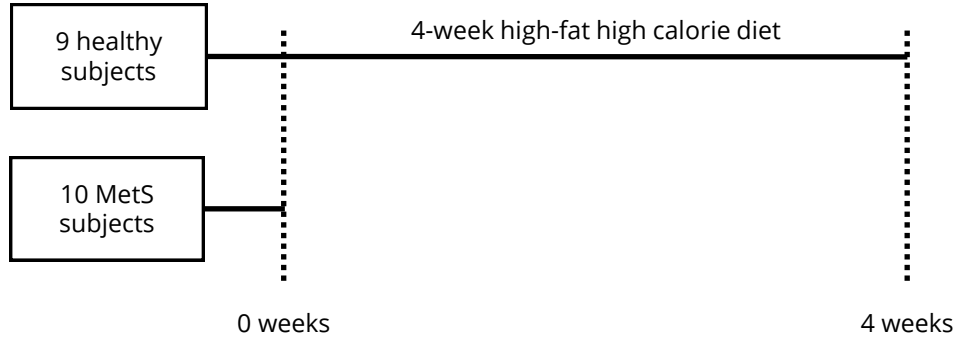

b High-fat challenge

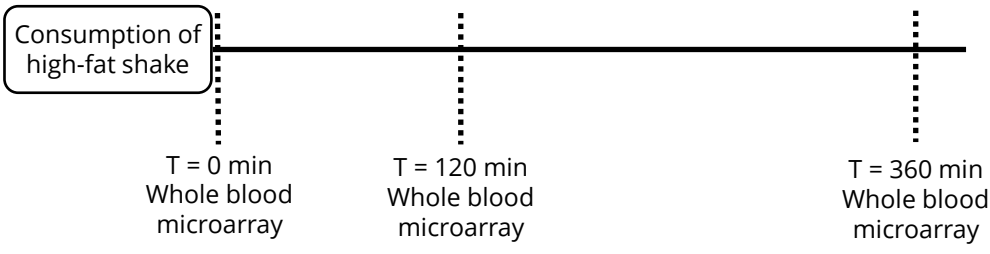

Figure 5-1. Study design and high-fat challenge.

Schematic overview of the study design (a) and high-fat challenge procedure (b). MetS, metabolic syndrome. 


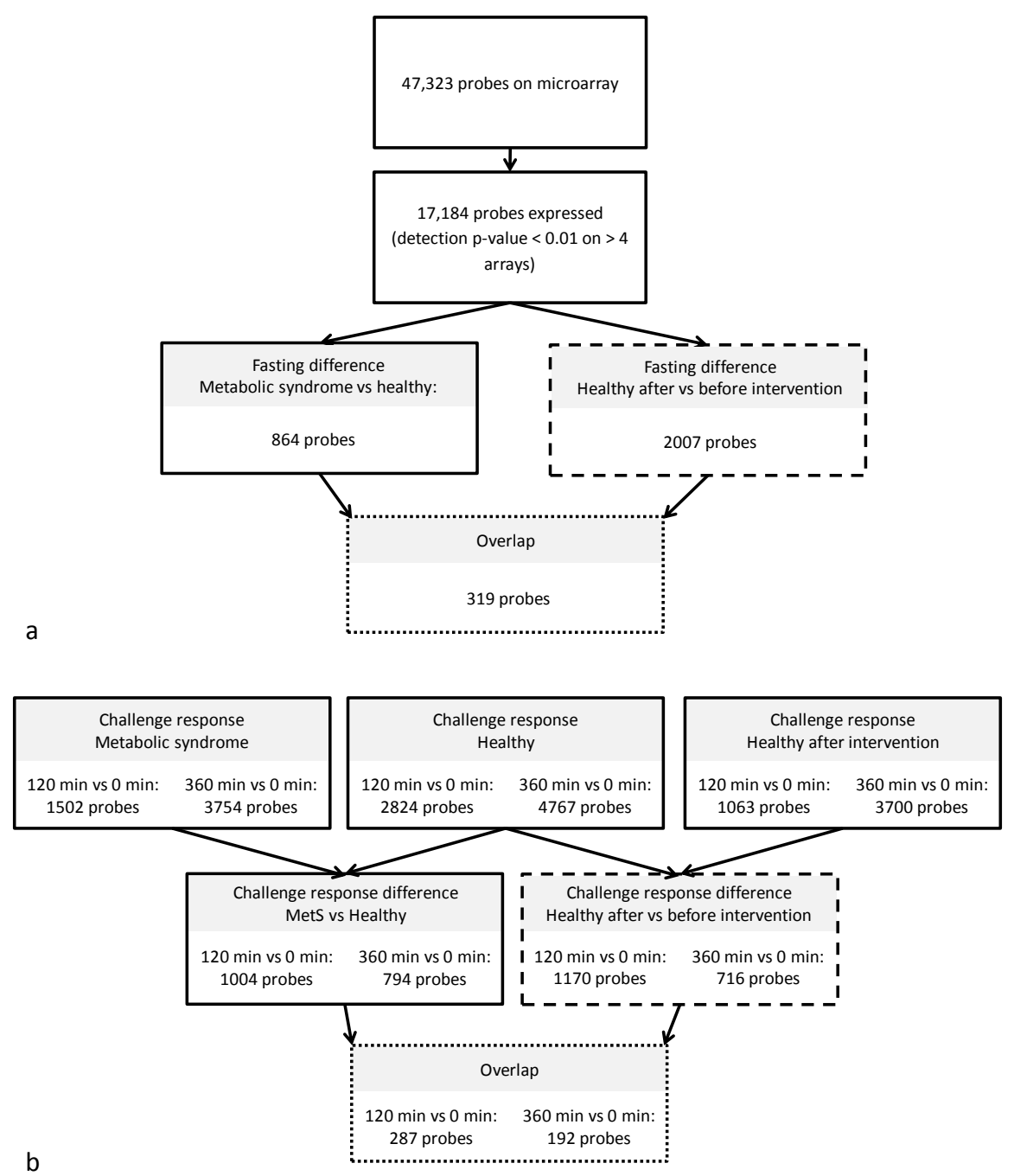

Figure 5-2. Probe selection workflow and number of differentially expressed genes.

Flowchart showing (a) probe selection workflow and number of differentially changed genes at fasting and (b) postprandially. Results from the dashed (- - -) panels were used to examine the additional value of studying whole genome gene expression changes in response to a high-fat challenge compared to studying only fasting gene expression profiles. Results from the dotted (...) panels, were used to examine whether the 4 week high-fat high-calorie diet can induce a shift in gene expression profiles in the healthy subjects towards more MetS-like profiles, both at fasting and post-prandially. 


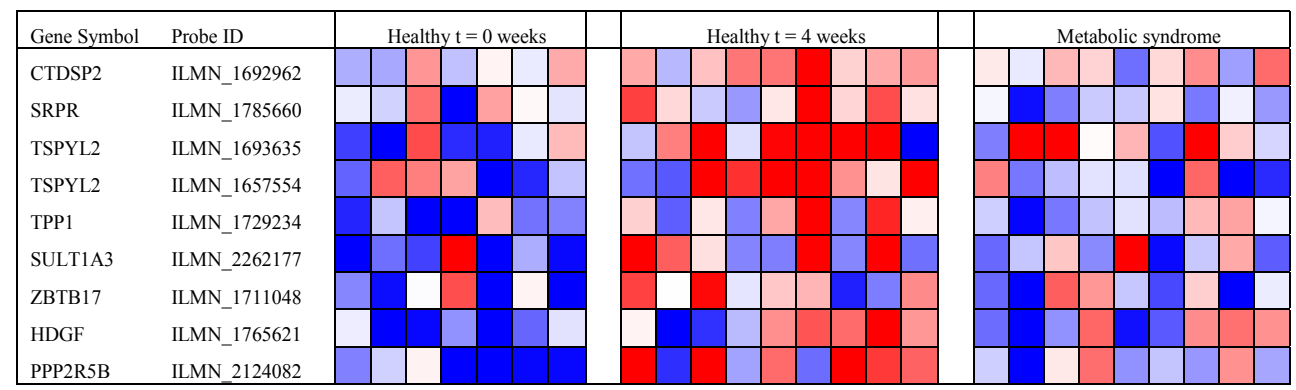

Figure 5-3. Heatmap showing individual high-fat challenge responses for genes in the chaperone gene activcation pathways at $\mathbf{1 2 0} \mathbf{m i n}$.

Genes were significantly differentially expressed when comparing the challenge response in the healthy group after intervention to before intervention. As a result, after intervention, the challenge response was similar to the response in the MetS subjects.

\begin{tabular}{|c|c|c|c|c|}
\hline Gene Symbol & Probe ID & Healthy $\mathrm{t}=0$ weeks & Healthy $t=4$ weeks & Metabolic syndrome \\
\hline TBK1 & ILMN_1739967 & & & \\
\hline $\mathrm{BIRC} 2$ & ILMN_2182704 & & & \\
\hline IRAK3 & ILMN_1913678 & & & \\
\hline CHUK & ILMN_1677041 & & & \\
\hline MAP3K1 & ILMN_1723020 & & & \\
\hline MEF2C & ILMN_1742544 & & & \\
\hline DHX9 & ILMN_1656016 & & & \\
\hline SARM1 & ILMN_1746265 & & & \\
\hline
\end{tabular}

Figure 5-4. Heatmap showing individual high-fat challenge responses for genes in the TLR cascade pathway at $\mathbf{3 6 0} \mathrm{min}$.

Genes were significantly differentially expressed when comparing the challenge response in the healthy group after intervention to before intervention. As a result, after intervention, the challenge response was similar to the response in the MetS subjects. 


\section{Meta-analysis of high-fat challenge-induced changes in blood cell whole genome gene expression}

Juri Matualatupauw, Helen Roche, Lydia Afman and Jildau Bouwman 


\section{Abstract}

\section{Background}

Several studies have examined the whole genome gene expression response in blood cells to high-fat challenges. However, changes in gene expression profiles described in these studies are quite diverse. We aimed to identify consistently up or downregulated genes and pathways in response to a high-fat challenge by using different integration and meta-analysis methods

\section{Methods}

Several methods to combine the data from the different studies were applied. Overlap in differentially expressed genes of each separate study were examined, P-values of each separate study were combined using Fishers methods and data were analyzed as one merged dataset. Significantly differentially expressed genes and pathways were compared between these methods.

\section{Results}

Selecting only the genes that were differentially expressed in all of the three separate studies resulted 67 differentially expressed genes, which were for a large part involved in circadian pathways. When using less strict methods, such as the Fishers P-value method and the merged dataset analysis, we observed changes in 1097 and 1182 genes, respectively. Overall, a high fat challenge upregulated pathways related to inflammation, whereas pathways related to unfolded protein response, protein processing, cholesterol biosynthesis and translation were downregulated upon a high-fat challenge.

\section{Discussion}

By performing this meta-analysis, we showed a general PBMC whole genome gene expression response to a high-fat challenge. Meta-analysis provides added value for the discovery of consistently differentially expressed genes and pathways compared to selecting only those genes and pathways that are identified in all separate studies. 


\section{Introduction}

Metabolic challenge tests are performed in nutritional research to magnify the small effects of nutrition on health (1). In addition, an individual's response to such a challenge is considered to be a marker of phenotypic flexibility and reflect the individual's health status. A type of metabolic challenge test that is frequently used in nutrition studies is the high-fat challenge, in which subjects consume a high-fat load containing 50-100 grams of fat. These high-fat challenges have been shown to not only affect postprandial lipid metabolism, but also other processes, such as inflammation, stress response, vascular health and glucose metabolism (2).

A good source of cells to study the abovementioned processes are peripheral blood mononuclear cells (PBMCs), which have proven useful for the determination of the effects of nutritional intervention on gene expression profiles (3-6). These cells are a subset of circulating white blood cells, which are non-invasive to collect during human intervention studies as they can be easily isolated from blood. Several studies have examined the effects of acute high-fat challenges on PBMC whole genome gene expression by comparing post-prandial gene expression profile responses to challenges with different compositions. We found that a high-SFA challenge induced expression of liver X receptor signaling genes, whereas a highPUFA challenge caused a downregulation of these genes (Bouwens et al. (7)). Matone et al. (8) studied the effects of a high-MUFA challenge and observed increased expression of inflammatory genes in several pathways, including Toll-like receptors, interleukins, TNF and NF- $\kappa B-$ related genes. In another study (Esser et al. (9)), we found that a high-SFA challenge decreased expression of cholesterol biosynthesis and uptake genes and increased cholesterol efflux genes compared to a high-MUFA challenge which increased expression of inflammatory genes and PPAR- $\alpha$ target genes involved in $\beta$-oxidation. We also performed a study (Chapter 5 ) in which we examined the effect of a high-SFA challenge on whole blood gene expression. We observed an increase in expression of several pathways including inflammatory pathways such as Toll-like receptors and TNFa signaling. These effects were only observed in healthy subjects and not in subjects with the MetS. Taking all these findings together, the changes in gene expression profiles described in these studies are quite diverse. Therefore, the question is raised if a general response to such a high-fat challenge, independent of fatty acid composition, BMI, sex, or age, can be characterized.

In this manuscript, our first aim was perform and compare different methods of combining the results of the abovementioned studies that examined the PBMC gene expression response 
to high-fat challenges. Secondly, using this cross-study meta-analysis, our goal was to identify consistently up or downregulated genes and pathways in response to a high-fat challenge. Ultimately, this may help to increase our understanding of how the body responds to a highfat load.

\section{Methods}

\section{Study design}

The studies by Bouwens et al., Matone et al. and Esser et al. were previously published and data is publicly available (7-9) (GSE13466, GSE56609 and GSE53232). These studies used single-channel Affymetrix microarrays to perform gene expression measurements, whereas Matualatupauw et al. (Chapter 5) used llumina HumanHt12-v4 Expression Beadchips. The studies by Bouwens et al. and Esser et al. applied a crossover design to examine highSFA versus high-MUFA and high-PUFA challenges respectively. To decrease heterogeneity between studies, we elected to analyze only the high-SFA challenges from both of these studies in this meta-analysis. Moreover, of the Matualatupauw et al. study (Chapter 5), we used only the high-fat challenge data that was acquired before the 4-week high-fat high-calorie diet intervention. The designs of the four studies are summarized in Table 6-1.

\section{Pre-processing}

Pre-processing was done for each study separately. For the studies using Affymetrix microarrays, raw CEL files were normalized using the Robust Multi-array Average algorithm (10), as implemented in the oligo R-package (11). A custom annotation was used based on reorganized oligonucleotide probes, which combines all individual probes for a gene (brainarray CDF files (ENTREZG v20)). We filtered out low expressing probes genes using Universal exPression Codes with a 0.5 cut-off, corresponding to a $50 \%$ likelihood that a gene is expressed (12).

For the study using Illumina beadarrays, pre-processing methods were described in detail previously (Chapter 5). Briefly, probe expression values were calculated using the neqc algorithm from the limma package (13). Low expressing probes were filtered by selecting only the probes with a detection p-value $<0.01$ in at least 5 arrays for further analysis. To end up with only one value per Entrez gene, we selected the probe with the highest variation when multiple probes were present for a gene. 
After filtering, only the genes present in all datasets, which were 6043 genes, were retained for further analysis. Initial analysis showed that the Matualatupauw et al. study showed a large batch effect when examining per subject $\log 2$-ratio (Figure 6-1). Therefore, it was decided to exclude this study from the analysis. The three remaining studies by Bouwens et al., Matone et al. and Esser et al. had an overlap of 7406 genes, which were used for further analysis. The workflow is summarized in Figure 6-2.

\section{Differentially expressed genes}

We first calculated log2-ratios per subject between the intensities before and after the challenge. These log2-ratios were used for further analysis using the Linear Models for Microarray data (limma) R-package. To identify differentially expressed genes, empirical Bayes moderated t-tests as implemented in limma were performed in each dataset separately as well as in the combined dataset of all studies. P-values were adjusted using Benjamini and Hochberg false discovery rate (14) and a Q-value $<0.05$ was considered significant.

In addition to the P-values per study and the p-values of the combined dataset, we also used Fisher's combined probability test to pool P-values from the three separate studies. We used the MetaDE R-package to calculate a test statistic (Chi2), which follows a chi distribution, and P- and FDR-values for each gene (15). An FDR Q $<0.05$ was used as significance cutoff, indicating that the corresponding gene was differentially expressed in at least one study.

\section{Pathway analysis}

For all pathway analyses, we used a list of gene sets that was derived by combining all gene sets taken from the Biocarta, KEGG, Reactome and Wikipathways databases. We performed gene set enrichment analysis (GSEA; http://www.broad.mit.edu/gsea) (16). Genes were ranked based on the moderated t-statistic and analyzed for over- or underrepresentation in the gene sets. GSEA was performed in each separate study as well as in the combined dataset. Gene sets with an FDR $\mathrm{q}<0.25$ were considered significantly enriched.

\section{Results}

In this study, we reanalyzed data of four separate studies of which the study designs are described in Table 6-1. Baseline characteristics of the study populations can be found in Table 6-2. Population differed in age, weight, height and BMI. To examine the presence of a potential batch effect between studies, we performed PCA on the per subject change in gene expression 
depicted as $\log 2$-ratios (Figure 6-1). The study of Matualatupauw et al. (Chapter 5) showed a separation from the other three studies, indicating the presence of large differences in gene expression changes between this study and the others. Therefore, the analysis was continued using the Bouwens et al., Matone et al. and Esser et al. studies only.

In Figure 6-2, the number of genes after filtering out non-expressed genes per data set/ study and the overlapping genes between datasets are shown. Combining the three datasets resulted in 7406 genes that were expressed in all datasets and these were used for further analysis. To examine variation within and between the datasets, we performed a PCA on the normalized $\log 2$ intensity values. This showed a clear separation between datasets (Figure 6-3A), reflecting batch effects. When the PCA was performed using per subject log2-ratios of the postprandial effects, datasets were found to be intermixed (Figure 6-3B). We continued our analyses using these $\log 2$-ratios.

\section{Differentially expressed genes}

To find the genes that showed a significant change in expression by the high-fat challenge in each individual dataset, we analyzed the data per study. An FDR Q $<0.05$ was used as significant cut-off value (Figure 6-4A). When comparing, the results of these analyses across datasets, 202 genes were found to be differentially expressed in at least two of the three datasets and 67 genes were found to be changed in all three datasets (Figure 6-4A). Figure 6-5 shows a heatmap of the postprandial gene expression changes in all subjects of the 67 genes that were differentially expressed in all studies.

Another method for microarray dataset integration that was applied, involved calculating an overall combined p-value based on p-values of each individual dataset analyzes using Fisher's method. We found 1097 differentially expressed genes at FDR Q $<0.05$ (Figure 6-4A).

The third method that we performed, was the creation of one merged dataset containing $\log 2$-ratios of all subjects from the three datasets. Statistical analysis was performed on this merged dataset, which revealed that 1182 genes were differentially expressed by the high-fat challenge at FDR $\mathrm{Q}<0.05$. Overlap of the genes identified using Fisher's method and the merged dataset analysis was 881 genes, as shown in Figure 6-4B. Overlap in genes between all comparisons is shown in Supplementary Figure S6-1.

\section{Gene set enrichment analysis}

To assess which pathways were upregulated by a high-fat challenge, we performed GSEA on 
the three separate datasets as well as on the merged dataset. Results are summarized in Table 3. Pathways related to circadian rhythm were found to be downregulated in each separate study as well as in the merged dataset. In the merged dataset, pathways related to interferon signaling were upregulated, whereas pathways related to circadian rhythm, unfolded protein response, protein processing, cholesterol biosynthesis and translation were downregulated. The individual significant postprandial gene expression changes of genes from these pathways are shown in a heatmap in Figure 6-6.

\section{Discussion}

We performed a cross-study meta-analysis of three studies that examined the PBMC gene expression response to a high-fat challenge using Affymetrix microarrays. Selection of the genes that were differentially expressed in the three separate analyses resulted in 67 differentially expressed genes, which were for a large part involved in circadian pathways. When using less strict methods, such as the Fishers P-value method and the merged dataset analysis, we observed changes in 1097 and 1182 genes, respectively. Pathways related to interferon signaling were upregulated, whereas pathways related to unfolded protein response, protein processing, cholesterol biosynthesis and translation were downregulated upon a highfat challenge.

Initially, we also included a study that used Illumina beadarrays measure gene expression in whole blood. As this study showed a large deviation from the others it was left out off further analysis. We have to two potential explanations for the large separation between the Matualatupauw et al. study and the other three studies. Firstly, this study used Illumina BeadArrays, whereas the others used Affymetrix GeneChips. This difference in microarray platform could have induced the bias (17), though it has been shown that measurements derived from both platforms show high agreement, especially in genes that are differentially expressed in a comparison $(18,19)$. Besides microarray platform, this study also differed in the source of RNA, which was whole blood compared to PBMCs in the others three studies. Whole blood contains RNA from all cell types present in blood including granulocytes (neutrophils, eosinophils, basophils), lymphocytes and monocytes, whereas PBMC RNA samples contain RNA from lymphocytes and monocytes only (20). One study compared differences in gene expression profiles in whole blood versus PBMCs in patients with advanced heart failure versus age-matched controls (21). They observed a considerable overlap and concordance in 
gene expression profiles between the two RNA sources, though they also observed differences. Differences in gene expression caused by different blood cell populations were also observed in another study (20). In this study, they observed lower signal-to-noise ratios and larger variability in whole blood compared to PBMCs. In summary, differences in microarray platforms and cell populations may potentially explain the large separation between the whole blood compared to the PBMC studies.

We observed consistent up or downregulation of several genes involved in circadian rhythm in the three studies. The studies used a similar protocol for their high-fat challenges. Subject arrived fasted early in the morning and consumed a high-fat challenge. PBMC blood samples were taken just before challenge consumption and 4 or 6 hours after consumption. In this regard, the times at which fasting and postprandial samples were taken can be expected to be similar between studies. In line with our findings, circadian oscillations in gene expression of PER1 and PER2 have been observed previously in PBMCs of healthy young men (22). In addition, PER1 and PER2 gene expression have been shown to peak shortly after awakening (23) or early in the morning (24), followed by a gradual decrease in expression in the subsequent hours. These findings coincide with the decrease in gene expression that we observed for these genes 4 and 6 hours after consumption of the high-fat challenge.

From studies in rodents, it is clear that many genes, including some involved in metabolic control and immune responses, show a circadian oscillations in gene and protein expression during the day (25-27). Interestingly, the IRE1 a pathway, which is part of the unfolded protein response was found to be under circadian control in mice, as observed from a 12 hour period rhythmic activation of target genes (28). Therefore the downregulation of pathways related to the unfolded protein response that we observed may potentially be caused by circadian oscillations as well, although studies showing this relationship in humans are lacking. In this study, we do not have a control group available and, therefore we are not able to distinguish between high-fat-induced and circadian oscillations changes in gene expression. The individual studies did include multiple challenges as a control and compared differences between them. Our analyzes of only one of the challenges per study clearly demonstrate the importance of a control group in metabolic challenge studies for the identification of actual challenge-induced effects on gene expression.

Another finding was the upregulation in expression of inflammation-related genes by the high-fat challenges. Triglyceride-rich lipoproteins and lipoprotein remnants are thought to play an important role in mediating postprandial inflammation (29). In the postprandial 
circulation, these lipoprotein may cause the activation of the nuclear factor $\mathrm{\kappa B}$ transcription factor, either by internalization of these lipoproteins by leukocytes (30), or by activating intracellular signaling by binding to CD14 and activation of toll-like receptor- 4 on the cell surface (31). These may be potential mechanism by which a high-fat challenge may cause an increase in inflammatory gene expression in PBMCs.

A postprandial downregulation was observed for genes related to cholesterol biosynthesis. Interestingly, this down regulation appears to mostly be attributed to changes in the study by Esser et al. and Bouwens et al and to a lesser extend the study by Matone et al. These differences may be caused by the high-SFA load in the first two studies compared to the high-MUFA load in the Matone et al. study. In the original study by Esser et al., a highMUFA challenge was also included, which did not cause changes in cholesterol biosynthesis genes, in accordance with our observations in the Matone et al. study. Furthermore, another similarity between these two high-MUFA challenges was an increase in expression of PPAR signaling pathways, which was observed in both. Expression of cholesterol biosynthesis genes is regulated via the SREBP-2 transcription factor through a negative feedback system (32), suggesting that intracellular cholesterol concentrations may be high after a high-SFA challenge and not after a high-MUFA challenge. The cause of this potential cholesterol concentration increase is unclear, as cholesterol biosynthesis gene expression changes appeared to be the largest in the study by Esser et al. even though the high-fat challenge that was used did not contain cholesterol, unlike the challenge used by Bouwens et al. that did contain cholesterolcontaining butter. Potentially, the higher SFA-load, the more at risk population of the Esser et al. vs. the Bouwens et al. study or the larger study population of Esser et al. may be responsible for the significant finding in the individual study of Esser al. on cholesterol biosynthesis gene expression .

A strong study-related difference in gene expression was observed (Figure 3A) when comparing $\log 2$-intensity values between studies, which is consistent with observations in many previous studies and is a commonly faced problem when combining different batches of microarray data (17). Several methods for correction of these batch-effect have been suggested $(17,33)$. Problematic with these approaches is that it is difficult to separate technical batch effects, such as batch effects caused by differences in PBMC collection or lab methodology, from biological effects (17). In our studies, differences in subject characteristics (age and BMI) or high-fat challenge composition are examples of factors that may cause batch effects. However, when we examined within-subject $\log 2$-ratios, the study-related batch effects disappeared, 
showing a large similarity in the responses to the high fat challenge across studies. This is an advantage of the within-subject design of the studies, as microarrays were performed on samples taken before as well as after the high-fat challenges in each individual. Furthermore, we found that the overlap in differentially expressed genes between the combined dataset analysis and the Fisher P-value combination method was large, with an overlap of $80 \%$. From these observations, we conclude that the between study batch effects are not a major source of variation in our studies and, therefore, we expect that batch effect correction would not be beneficial. Nevertheless, many differences exist between the three studies, which include differences in study population, challenge composition, time of the post-prandial measurement, microarray platform and laboratory methods. These are all potential sources of variability in our analyzes and may have decreased our power to detect high-fat challengeinduced changes in gene expression. Consequently, we may have only picked up the genes and pathways with the strongest changes. In the future, minimizing differences between studies by increased standardization in methods, including the use of a standardized metabolic challenge as described by Stroeve et al. (2), the consumption of a standardized low-fat meal the evening before a challenge test, and using RNA from the same cell sources would greatly enhance power of these types of meta-analyses.

To our knowledge, this is the first study that has performed a meta-analysis of microarray datasets from several acute nutritional challenge intervention studies. We used several metaanalysis approaches to identify changes in PBMC gene expression upon a high-fat challenge. Several differences exists between the studies, such as microarray platform, number and characteristics of subjects, high-fat challenge composition, laboratory where the analysis took place and timepoint of the postprandial measurement. Nevertheless, the identified changes are consistent across datasets, demonstrating that this may be a valuable approach that can also be applied in future studies.

Of the used methods, selection of genes that were differentially expressed in all three separate studies was the strictest, resulting in low number of differentially expressed genes that did show the most consistent and robust regulation in all studies. When using less strict methods, such as the Fisher P-value method and the merged dataset analysis, we observed changes in additional genes and pathways.

In conclusion, meta-analysis provides added value for the discovery of consistently differentially expressed genes and pathways compared to selecting only those genes and pathways that are identified in all separate studies. Using meta-analyzes of PBMC whole genome gene 
expression responses to high-fat challenges of studies varying in study population, challenge composition and research laboratory, we identified a general PBMC whole genome gene expression response to a high fat challenge.

\section{References}

1. van Ommen B, Keijer J, Heil SG, Kaput J. Challenging homeostasis to define biomarkers for nutrition related health. Molecular nutrition \& food research. 2009;53(7):795-804.

2. Stroeve JH, van Wietmarschen H, Kremer BH, van Ommen B, Wopereis S. Phenotypic flexibility as a measure of health: the optimal nutritional stress response test. Genes \& nutrition. 2015;10(3):459.

3. Bouwens M, van de Rest O, Dellschaft N, Bromhaar MG, de Groot LC, Geleijnse JM, et al. Fish-oil supplementation induces antiinflammatory gene expression profiles in human blood mononuclear cells. The American journal of clinical nutrition. 2009;90(2):415-24.

4. Myhrstad MC, Ulven SM, Gunther CC, Ottestad I, Holden M, Ryeng E, et al. Fish oil supplementation induces expression of genes related to cell cycle, endoplasmic reticulum stress and apoptosis in peripheral blood mononuclear cells: a transcriptomic approach. J Intern Med. 2014;276(5):498-511.

5. van Dijk SJ, Feskens EJ, Bos MB, Hoelen DW, Heijligenberg R, Bromhaar MG, et al. A saturated fatty acid-rich diet induces an obesity-linked proinflammatory gene expression profile in adipose tissue of subjects at risk of metabolic syndrome. The American journal of clinical nutrition. 2009;90(6):1656-64.

6. de Mello VD, Kolehmanien M, Schwab U, Pulkkinen L, Uusitupa M. Gene expression of peripheral blood mononuclear cells as a tool in dietary intervention studies: What do we know so far? Molecular nutrition \& food research. 2012;56(7):1160-72.

7. Bouwens M, Grootte Bromhaar M, Jansen J, Muller M, Afman LA. Postprandial dietary lipid-specific effects on human peripheral blood mononuclear cell gene expression profiles. The American journal of clinical nutrition. 2010;91(1):208-17.

8. Matone A, O'Grada CM, Dillon ET, Morris C, Ryan MF, Walsh M, et al. Body mass index mediates inflammatory response to acute dietary challenges. Mol Nutr Food Res. 2015;59(11):2279-92.

9. Esser D, van Dijk SJ, Oosterink E, Lopez S, Muller M, Afman LA. High fat challenges with different fatty acids affect distinct atherogenic gene expression pathways in immune cells from lean and obese subjects. Mol Nutr Food Res. 2015;59(8):1563-72.

10. Irizarry RA, Bolstad BM, Collin F, Cope LM, Hobbs B, Speed TP. Summaries of Affymetrix GeneChip probe level data. Nucleic acids research. 2003;31(4):e15.

11. Carvalho BS, Irizarry RA. A framework for oligonucleotide microarray preprocessing. Bioinformatics. 2010;26(19):2363-7.

12. Piccolo SR, Sun Y, Campbell JD, Lenburg ME, Bild AH, Johnson WE. A single-sample microarray normalization method to facilitate personalized-medicine workflows. Genomics. 2012;100(6):337-44.

13. Ritchie ME, Phipson B, Wu D, Hu Y, Law CW, Shi W, et al. limma powers differential expression analyses for RNA-sequencing and microarray studies. Nucleic acids research. 2015;43(7):e47.

14. Benjamini Y, Hochberg Y. Controlling the false discovery rate: a practical and powerful approach to multiple testing. Journal of the royal statistical society Series B (Methodological). 1995:289-300.

15. Wang X, Kang DD, Shen K, Song C, Lu S, Chang LC, et al. An R package suite for microarray meta-analysis in quality control, differentially expressed gene analysis and pathway enrichment detection. Bioinformatics. 2012;28(19):2534-6. 
16. Subramanian A, Tamayo P, Mootha VK, Mukherjee S, Ebert BL, Gillette MA, et al. Gene set enrichment analysis: a knowledge-based approach for interpreting genome-wide expression profiles. Proceedings of the National Academy of Sciences of the United States of America. 2005;102(43):15545-50.

17. Leek JT, Scharpf RB, Bravo HC, Simcha D, Langmead B, Johnson WE, et al. Tackling the widespread and critical impact of batch effects in high-throughput data. Nature reviews Genetics. 2010;11(10):733-9.

18. Barnes M, Freudenberg J, Thompson S, Aronow B, Pavlidis P. Experimental comparison and cross-validation of the Affymetrix and Illumina gene expression analysis platforms. Nucleic acids research. 2005;33(18):5914-23.

19. Consortium M, Shi L, Reid LH, Jones WD, Shippy R, Warrington JA, et al. The MicroArray Quality Control (MAQC) project shows inter- and intraplatform reproducibility of gene expression measurements. Nature biotechnology. 2006;24(9):1151-61.

20. Joehanes R, Johnson AD, Barb JJ, Raghavachari N, Liu P, Woodhouse KA, et al. Gene expression analysis of whole blood, peripheral blood mononuclear cells, and lymphoblastoid cell lines from the Framingham Heart Study. Physiological genomics. 2012;44(1):59-75.

21. Bondar G, Cadeiras M, Wisniewski N, Maque J, Chittoor J, Chang E, et al. Comparison of whole blood and peripheral blood mononuclear cell gene expression for evaluation of the perioperative inflammatory response in patients with advanced heart failure. PloS one. 2014;9(12):e115097.

22. Boivin DB, James FO, Wu A, Cho-Park PF, Xiong H, Sun ZS. Circadian clock genes oscillate in human peripheral blood mononuclear cells. Blood. 2003;102(12):4143-5.

23. James FO, Boivin DB, Charbonneau S, Belanger V, Cermakian N. Expression of clock genes in human peripheral blood mononuclear cells throughout the sleep/wake and circadian cycles. Chronobiology international. 2007;24(6):1009-34.

24. Kusanagi H, Hida A, Satoh K, Echizenya M, Shimizu T, Pendergast JS, et al. Expression profiles of 10 circadian clock genes in human peripheral blood mononuclear cells. Neuroscience research. 2008;61(2):136-42.

25. Ribas-Latre A, Eckel-Mahan K. Interdependence of nutrient metabolism and the circadian clock system: Importance for metabolic health. Molecular metabolism. 2016;5(3):133-52.

26. Bass J, Takahashi JS. Circadian integration of metabolism and energetics. Science. 2010;330(6009):1349-54.

27. Eckel-Mahan KL, Patel VR, de Mateo S, Orozco-Solis R, Ceglia NJ, Sahar S, et al. Reprogramming of the circadian clock by nutritional challenge. Cell. 2013;155(7):1464-78.

28. Cretenet G, Le Clech M, Gachon F. Circadian clock-coordinated $12 \mathrm{Hr}$ period rhythmic activation of the IRE1alpha pathway controls lipid metabolism in mouse liver. Cell metabolism. 2010;11(1):47-57.

29. de Vries MA, Klop B, Eskes SA, van der Loos TL, Klessens-Godfroy FJ, Wiebolt J, et al. The postprandial situation as a pro-inflammatory condition. Clinica e investigacion en arteriosclerosis : publicacion oficial de la Sociedad Espanola de Arteriosclerosis. 2014;26(4):184-92.

30. Maziere C, Maziere JC. Activation of transcription factors and gene expression by oxidized low-density lipoprotein. Free radical biology \& medicine. 2009;46(2):127-37.

31. Miller YI, Viriyakosol S, Binder CJ, Feramisco JR, Kirkland TN, Witztum JL. Minimally modified LDL binds to CD14, induces macrophage spreading via TLR4/MD-2, and inhibits phagocytosis of apoptotic cells. The Journal of biological chemistry. 2003;278(3):1561-8.

32. Horton JD, Goldstein JL, Brown MS. SREBPs: activators of the complete program of cholesterol and fatty acid synthesis in the liver. The Journal of clinical investigation. 2002;109(9):1125-31.

33. Johnson WE, Li C, Rabinovic A. Adjusting batch effects in microarray expression data using empirical Bayes methods. Biostatistics. 2007;8(1):118-27. 


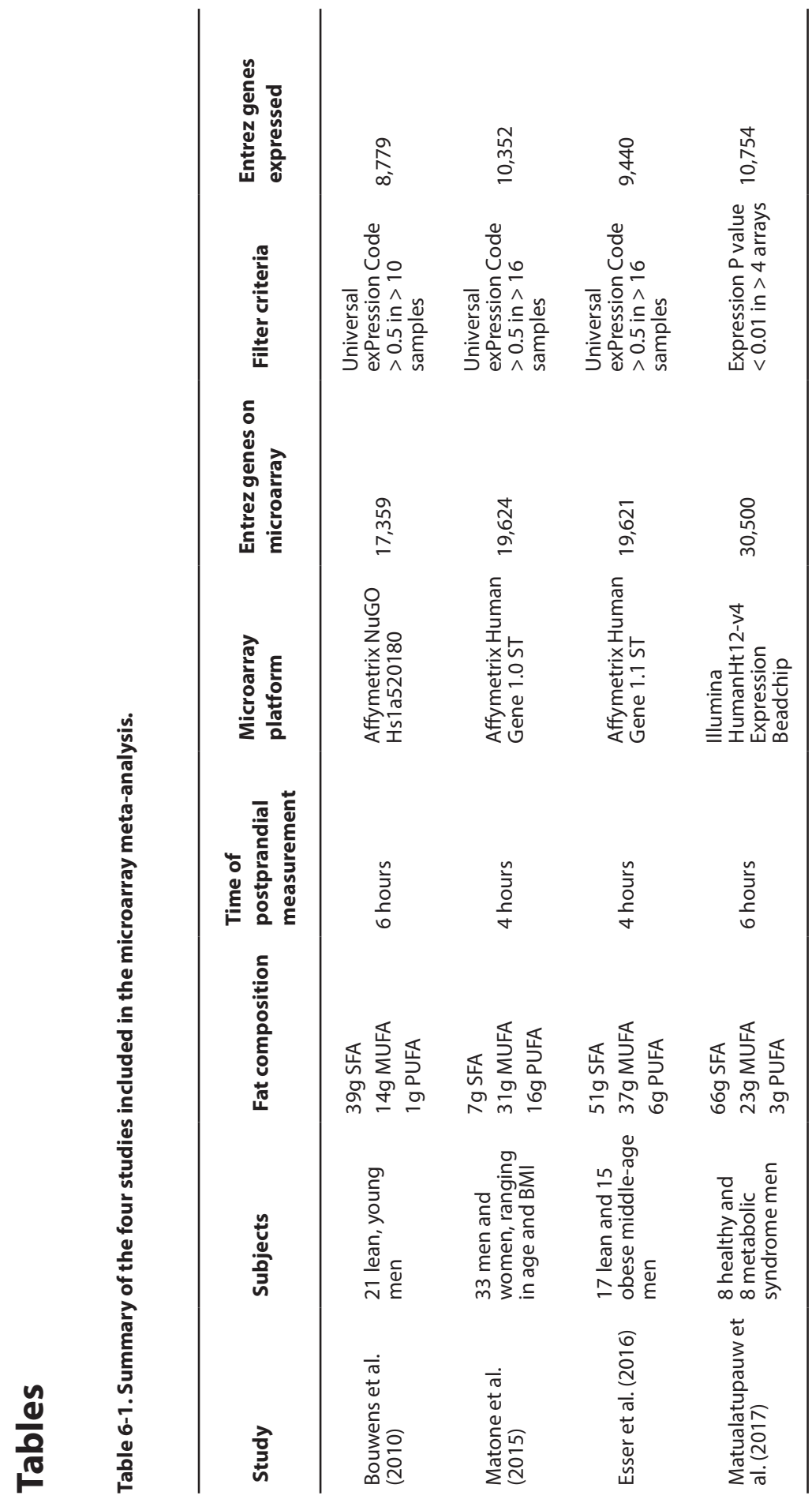


Table 6-2. Baseline characteristics of the four study populations.

\begin{tabular}{llllll}
\hline & Bouwens et al. & Matone et al. & Esser et al. & $\begin{array}{l}\text { Matualatupauw } \\
\text { et al. }\end{array}$ & P-value \\
\hline Age (years) & $21 \pm 3^{\mathrm{a}}$ & $37.3 \pm 13^{\mathrm{b}}$ & $62 \pm 5^{\mathrm{c}}$ & $46 \pm 7^{\mathrm{d}}$ & $<0.001$ \\
Weight $(\mathrm{kg})$ & $74.4 \pm 8.1^{\mathrm{a}}$ & $83.0 \pm 18.0^{\mathrm{b}}$ & $89.0 \pm 18.0^{\mathrm{b}}$ & $86.8 \pm 13.0^{\mathrm{b}}$ & 0.009 \\
Height $(\mathrm{m})$ & $1.84 \pm 0.06^{\mathrm{a}}$ & $1.75 \pm 0.09^{\mathrm{b}}$ & $1.78 \pm 0.07^{\mathrm{bc}}$ & $1.81 \pm 0.07^{\mathrm{ac}}$ & 0.001 \\
BMI (kg/m2) & $22.1 \pm 2.0^{\mathrm{a}}$ & $27.0 \pm 6.1^{\mathrm{b}}$ & $27.9 \pm 4.9^{\mathrm{b}}$ & $26.4 \pm 3.2^{\mathrm{b}}$ & $<0.001$ \\
\hline
\end{tabular}

Data are presented as mean $\pm \mathrm{sd}$. Differences between groups were determined using one-way ANOVA and corresponding P-values are shown. Different letters indicate differences between groups, as determined using LSD post-hoc tests.

Table 6-3. Summary of GSEA results.

\begin{tabular}{|c|c|c|c|c|}
\hline & Bouwens et al. & Matone et al. & Esser et al. & $\begin{array}{l}\text { Merged } \\
\text { dataset }\end{array}$ \\
\hline Interferon signaling & $\uparrow$ & $\uparrow$ & & $\uparrow$ \\
\hline Circadian rhythm & $\downarrow$ & $\downarrow$ & $\downarrow$ & $\downarrow$ \\
\hline Unfolded protein response & & $\downarrow$ & $\downarrow$ & $\downarrow$ \\
\hline mRNA Splicing & & & $\downarrow$ & \\
\hline Protein processing & & & $\downarrow$ & $\downarrow$ \\
\hline Cholesterol biosynthesis & & & $\downarrow$ & $\downarrow$ \\
\hline Translation & & $\downarrow$ & & $\downarrow$ \\
\hline Cell cycle & & $\downarrow$ & & \\
\hline Semaphorin processing & & & $\downarrow$ & \\
\hline Oxidative phosphorylation & & $\downarrow$ & & \\
\hline Toll-like receptor cascades & & $\uparrow$ & & \\
\hline PPAR signaling pathway & & $\uparrow$ & & \\
\hline
\end{tabular}

Differentially expressed gene sets were visualized using the Enrichment Map plugin in Cytoscape. Up and down regulated gene set clusters in each study and in the merged dataset were summarized in this table. 


\section{Figures}

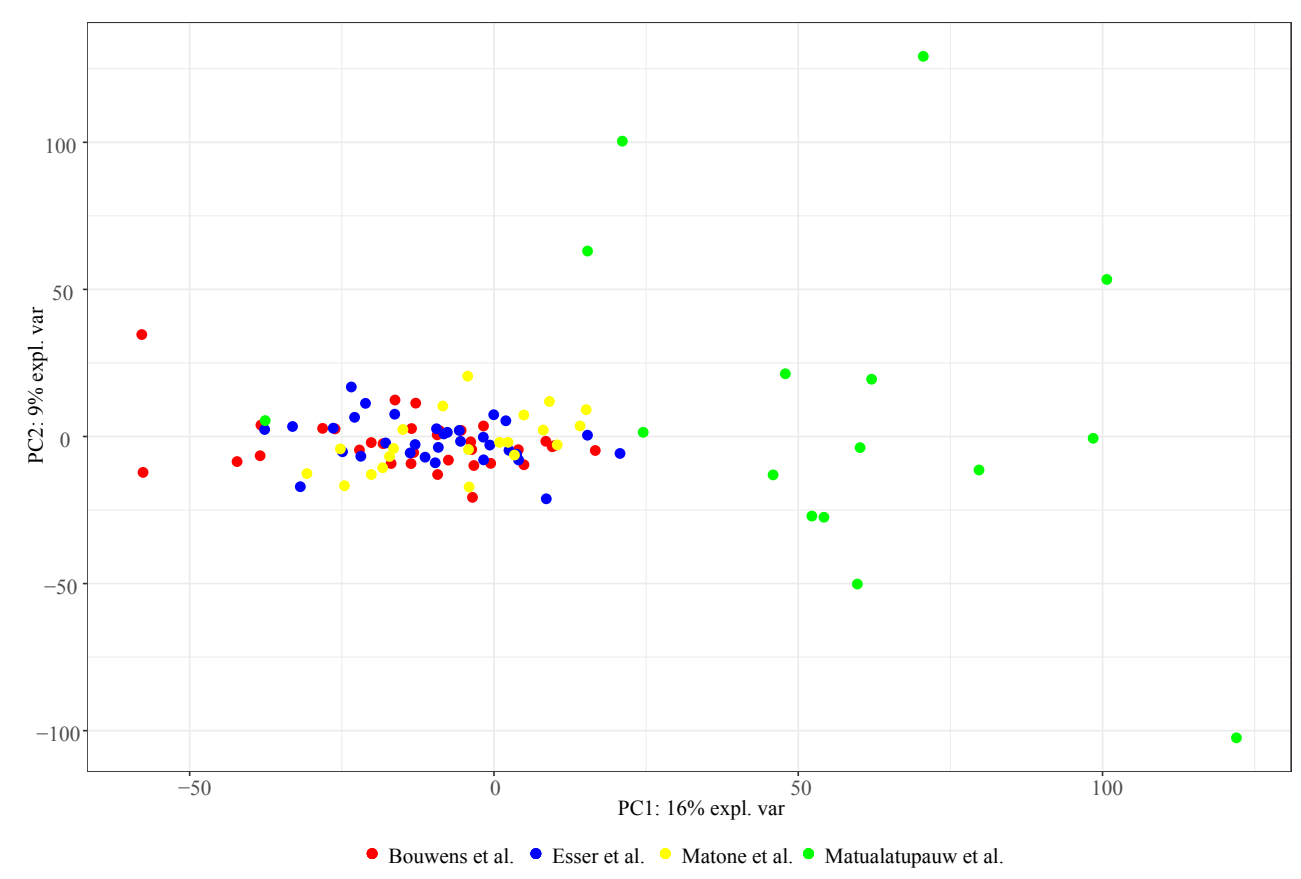

Figure 6-1. PCA of the log2-ratios of the four studies.

Every dot represents one subject. 


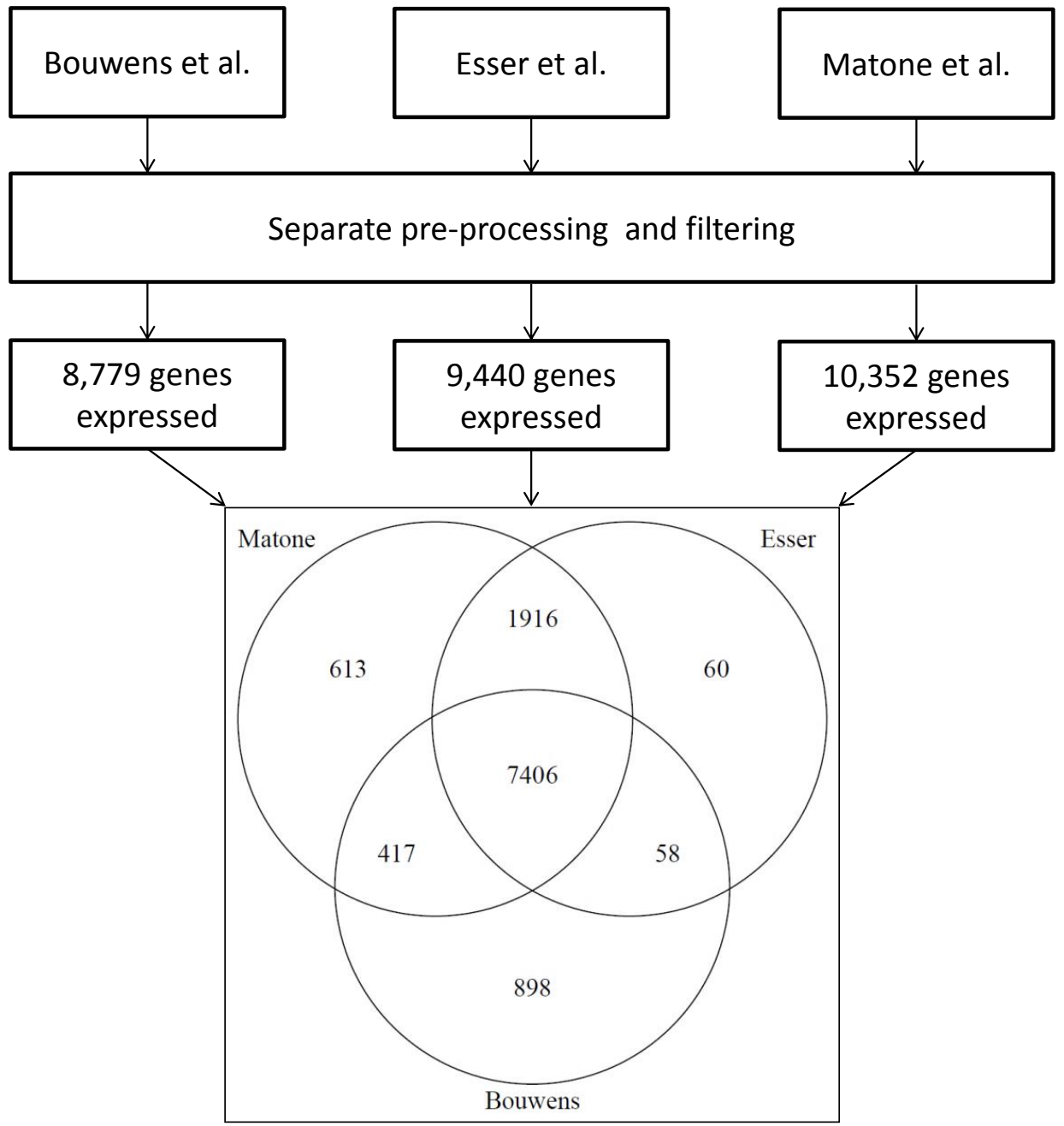

Figure 6-2. Gene selection workflow 
Meta-analysis of high-fat challenge microarray studies

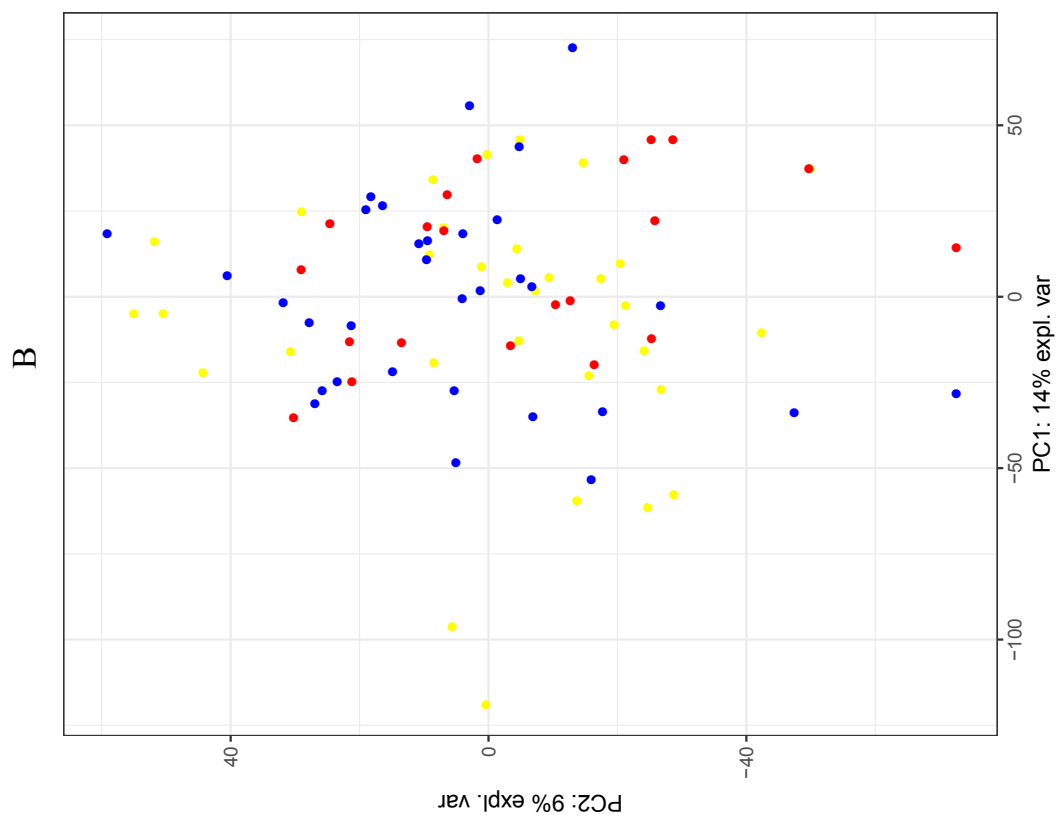

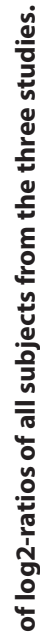

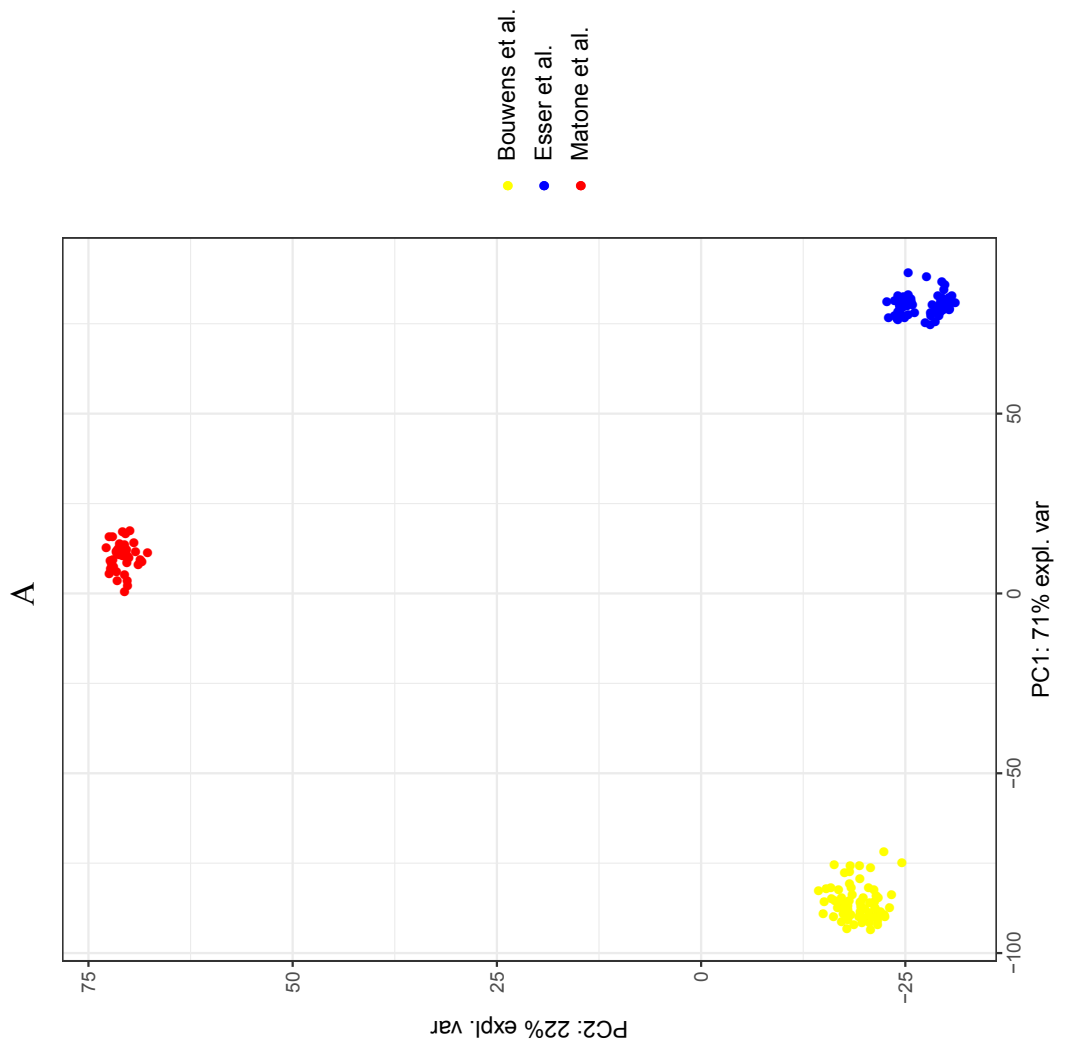


A

Bouwens et al.

Matone et al.

Esser et al.

Significant in all 3 studies

Significant in at least 2 studies

Fisher's method

Merged dataset analysis

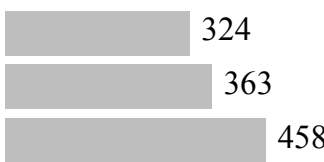

67

\section{2}

1097

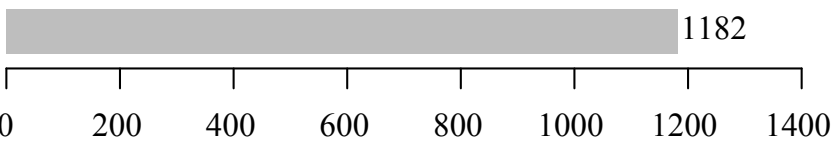

\# of differentially expressed genes

B

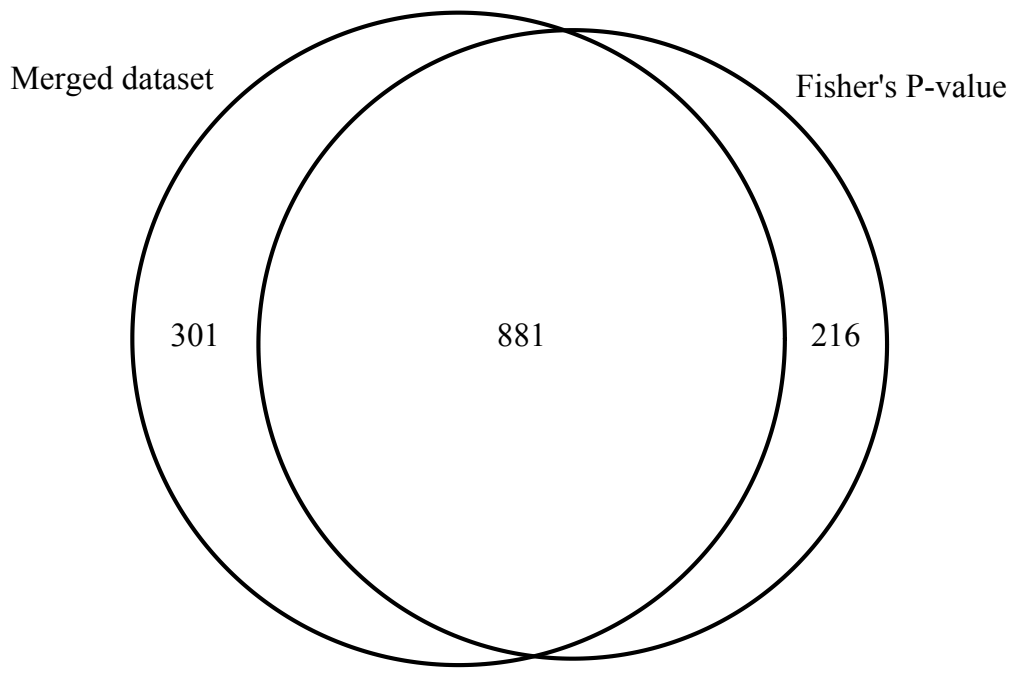

Figure 6-4. A. Number of significantly differentially expressed genes identified in each separate dataset and in the meta-analysis of the datasets using different methods. B. Venn diagram of overlap between Fishers method and the merged dataset analysis

Overlap in genes between all results are shown in Supplemental Figure S6-1. 


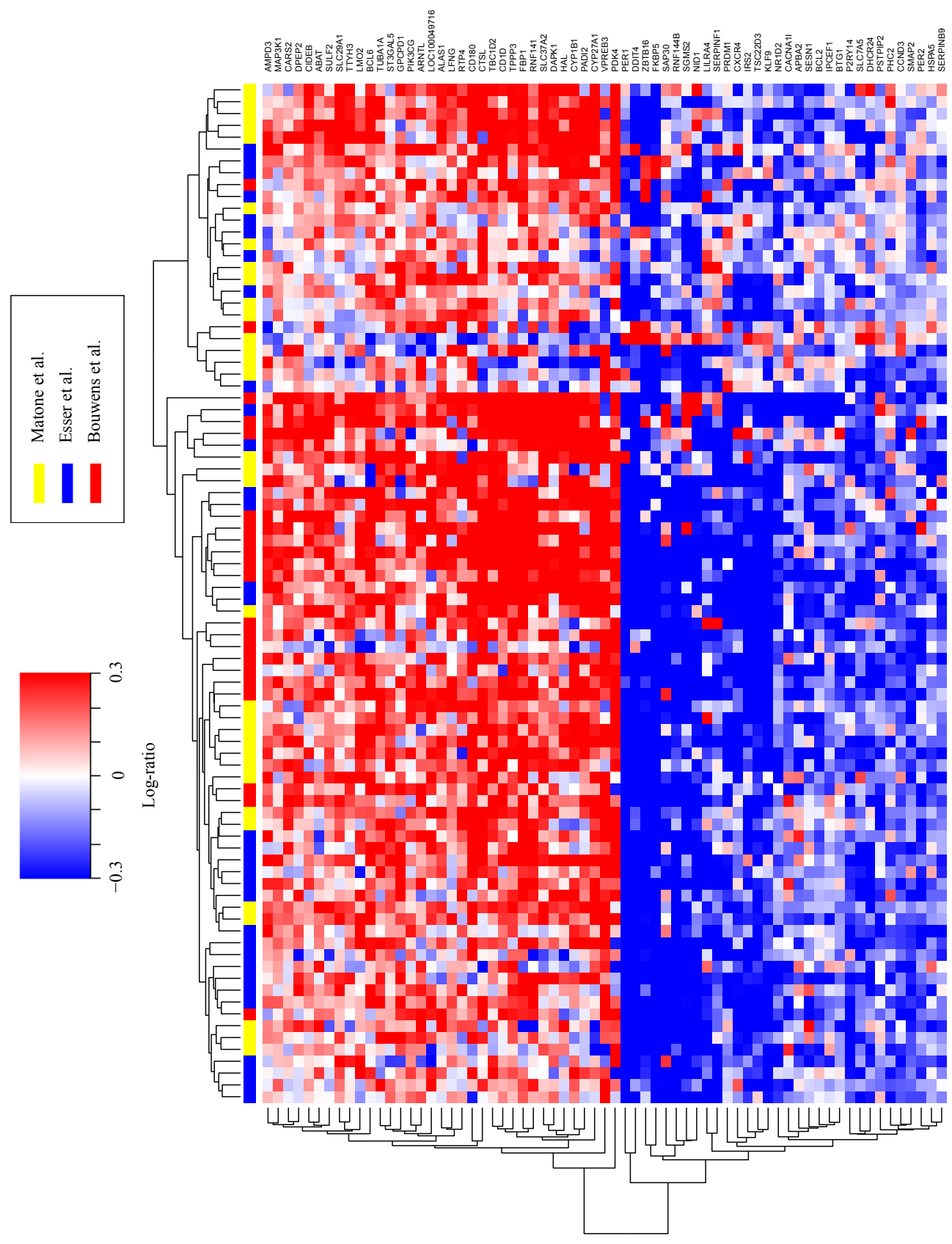

Figure 6-5. Heatmap depicting individual gene expression changes by a high-fat challenges of the 67 genes that are differentially expressed in all studies (FDR $Q<0.05$ ).

Log-ratios are shown for each gene in each subject of the three studies. 

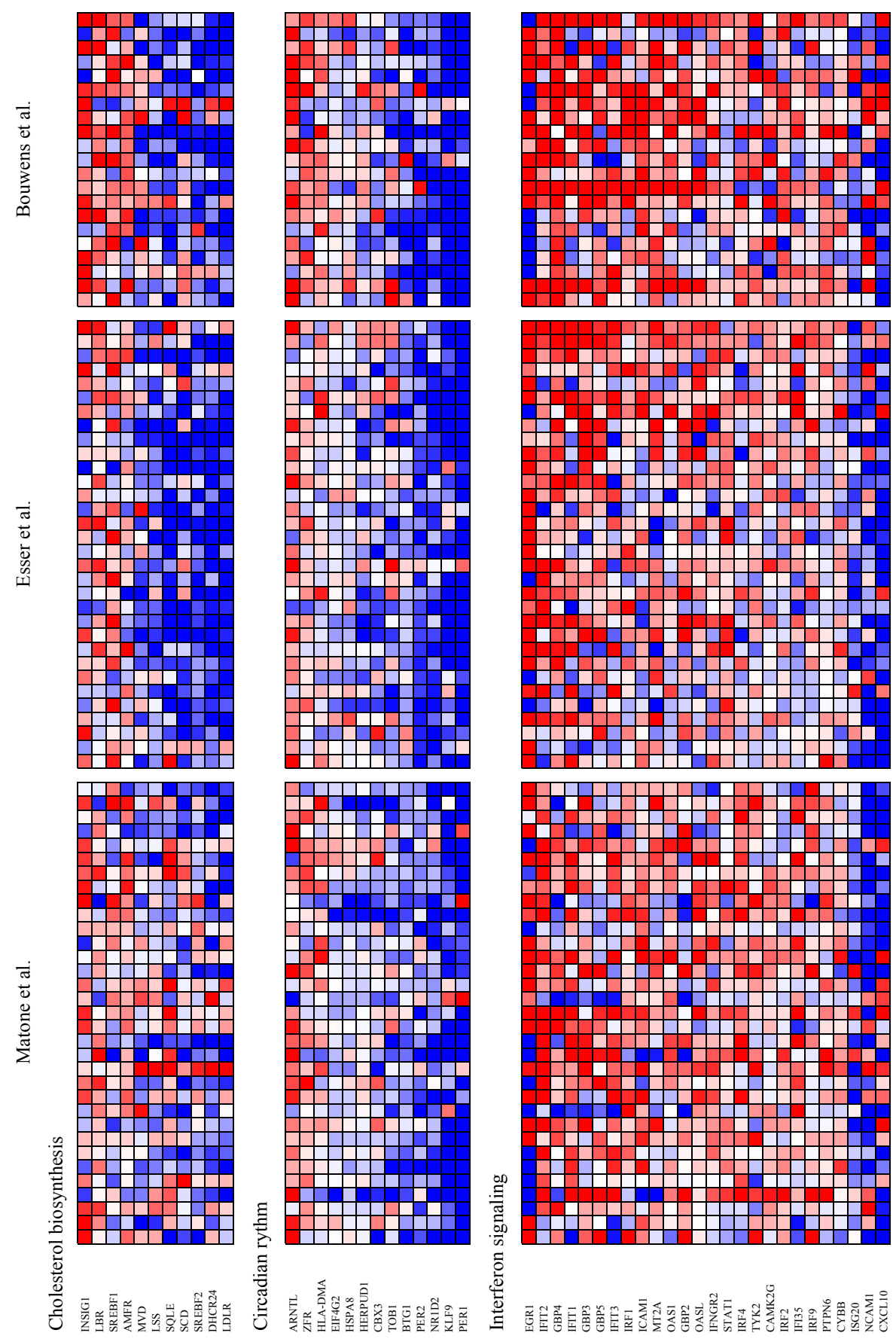


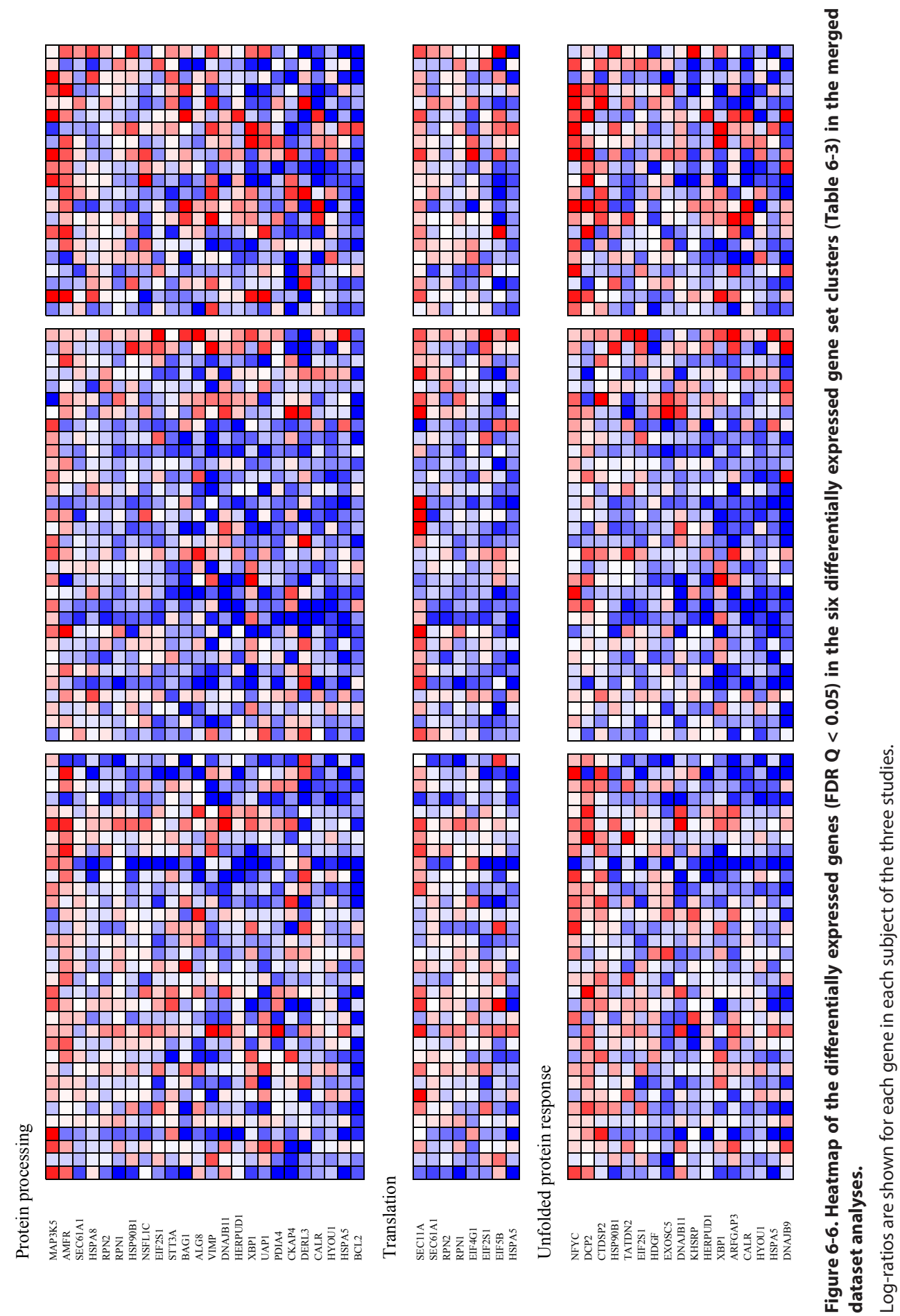




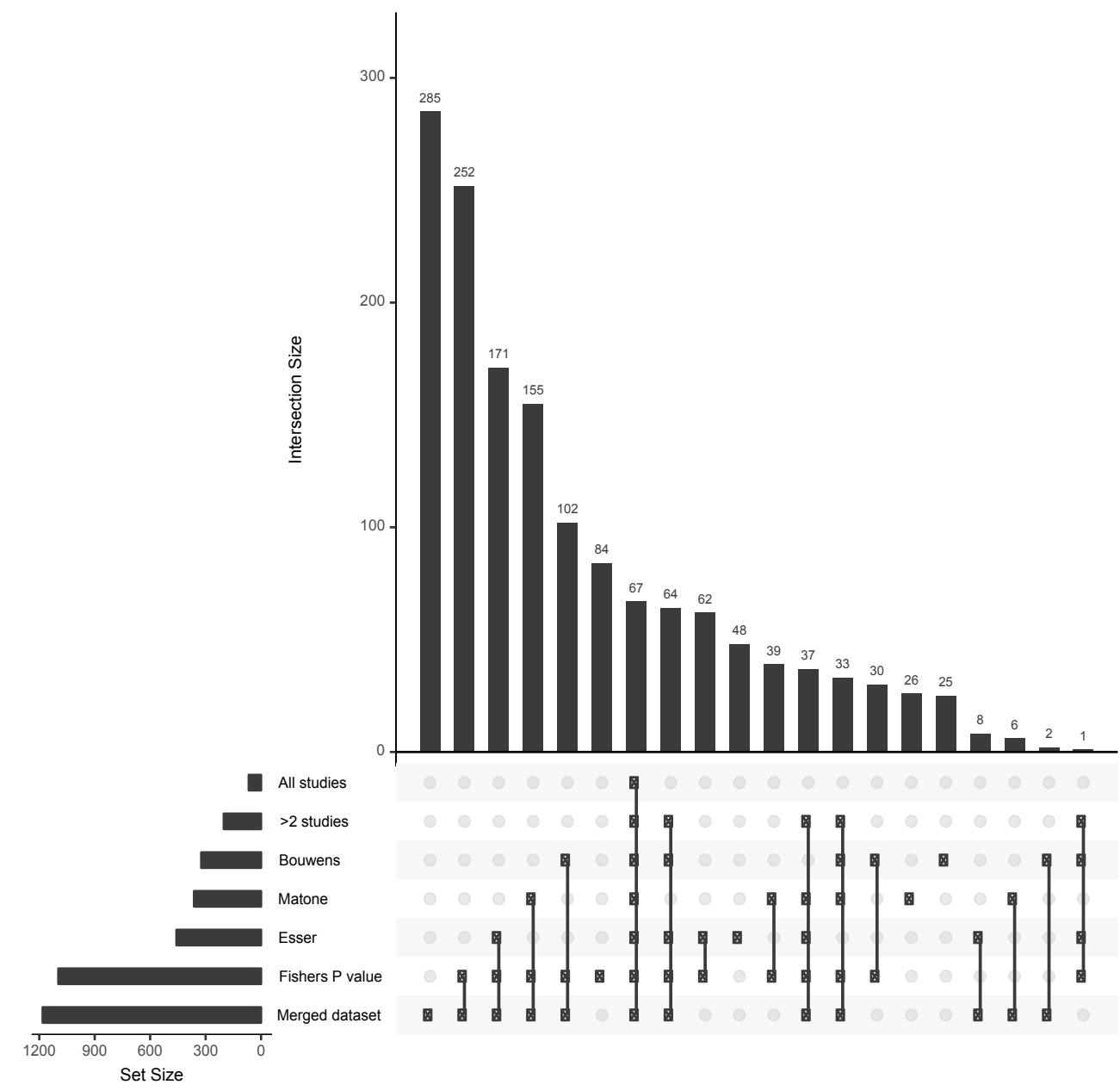

Supplementary Figure S6-1. Overlap in differentially expressed genes in separate studies and in different meta-analysis approaches 


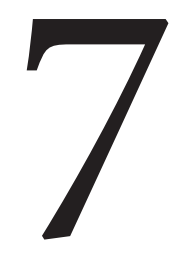

General discussion 


\section{Aim of the research}

Health effects of dietary fatty acids depend on the type of fatty acid that is consumed. The aim of this thesis was to increase our understanding of the molecular mechanisms underlying the effects of dietary fatty acids. To do this, we examined changes in whole genome gene expression profiles upon both acute as well as long-term dietary fatty acid interventions. Furthermore, from previous research, it is clear that large inter-individual differences in the response to dietary fatty acids exist (1-6). We used whole genome gene expression analyses to increase our understanding of the mechanisms underlying some of these inter-individual differences.

\section{Main findings of the research}

A summary of the main findings of the microarray analyses of each chapter can be found in

Table 7-1. The main findings in each chapter were:

- Transcriptome analyses can be applied to identify differences in response to dietary interventions based on phenotypic characteristics. In Chapter 2, we performed a review of all previously published studies that used microarrays to do this. The studies that are performed are low in number and are often secondary analyses of existing data.

- In Chapter 3, we studied the effects of the apolipoprotein E4 (APOE4) allele on gene expression profiles, at baseline and upon a long-term fish-oil supplementation intervention. APOE4 was associated with an increased expression of cholesterol biosynthesis and interferon signalling pathways, which may in part explain the increased CVD risk of APOE4. Fish-oil supplementation may be beneficial by decreasing interferon signalling-related gene expression in APOE4 carriers.

- In Chapter 4, we studied the effect of a 12-week high medium-chain saturated fatty acid diet on subcutaneous adipose tissue gene expression profiles. Intake of this diet increased expression of genes increased expression of genes involved in oxidative energy metabolism in subcutaneous adipose tissue, and decreased inflammation-related gene expression. These findings suggest that medium-chain saturated fatty acids have potentially beneficial effects on adipose tissue gene expression profiles.

- In Chapter 5, we studied whole genome gene expression changes in whole blood 
at fasting and upon a high-fat challenge. Fasting whole blood whole genome gene expression profiles are highly responsive to a 4-week high-fat high-calorie diet, showing changes in many pathways in the direction of a metabolic syndrome-like gene expression profile. High-fat challenge responses in healthy subjects show only small changes upon the dietary intervention and a minimal shift in the direction of the metabolic syndrome. This indicates that fasting gene expression profiles are more suitable for examining the subtle changes induced by a 4-week high-fat high-calorie diet.

- In Chapter 6, we combined microarray data from several previously published highfat challenge studies varying in study population, challenge composition and research laboratory. By performing this meta-analysis, we showed a general PBMC whole genome gene expression response to a high-fat challenge. Meta-analysis provides added value for the discovery of consistently differentially expressed genes and pathways compared to selecting only those genes and pathways that are identified in all separate studies.

\section{Long-term effects of dietary fatty acids}

In Chapter 3 and Chapter 4, we studied the gene expression responses in PBMCs and subcutaneous adipose tissue to long-term dietary interventions with fatty acids. The fish-oil supplementation intervention that was studied in Chapter 3 had already been shown to induce anti-inflammatory and anti-atherogenic changes in gene expression profiles (7). In this thesis, we dissected this further and showed anti-inflammatory effects of fish-oil supplementation in carriers of the APOE4 gene variant with a downregulation of interferon signalling pathways. In Chapter 4, we studied the effects of a dietary intervention consisting of products produced from milk that was high in medium-chain saturated fatty acids vs milk that was low in medium-chain saturated fatty acids. Similar to the fish-oil intervention (Chapter 3), we observed a downregulation of inflammation-related pathways, though in this case we did not measure in the blood, but in the adipose tissue. The high medium-chain saturated fatty acid diet (Chapter 4) caused changes in gene expression profiles with a decrease in inflammatory pathways, especially of the complement system, together with an increased expression of citric acid cycle and oxidative phosphorylation pathways.

The findings from Chapter 3 and Chapter 4 may be of relevance for the low-grade inflammation that is associated with obesity (8). This low-grade inflammation is characterized by infiltration of macrophages into the adipose tissue and a phenotypic switch of these 
macrophages towards the pro-inflammatory M1 phenotype (9). M1 macrophages are characterized by a reliance on glycolysis and the pentose phosphate pathway for their ATP production, whereas the anti-inflammatory M2 macrophages have a highly active citric acid cycle and oxidative phosphorylation (10). In accordance with the M2 response, the high MCSFA diet (Chapter 4) caused changes in gene expression profiles with an increased expression of citric acid cycle and oxidative phosphorylation pathways together with to a decrease in inflammatory pathways. Besides local adipose tissue inflammation, obesity can also lead to secretion of pro-inflammatory cytokines from the adipose tissue into the circulation (11). Circulating blood cells, such as PBMCs, exposed to these cytokines may play an important role in obesity-associated low-grade inflammation, as observed from an increased production of inflammatory cytokines and a decreased production of anti-inflammatory cytokines in PBMCs of obese subjects $(12,13)$. Furthermore, in atherosclerotic lesions, interferon- $\gamma$ produced by T-lymphocytes is highly expressed and affects many processes involved in the pathogenesis of atherosclerosis (14). The downregulation of interferon-related pathways observed in APOE4 carriers in Chapter 3 may be beneficial with regard to both these mechanisms. Taken together, we observed potentially beneficial changes in gene expression of the fish-oil supplementation intervention in PBMCs, and of the medium-chain saturated fatty acid intervention in adipose tissue.

Previously, strong similarities between PBMC and adipose tissue gene expression profiles have been observed, especially regarding genes involved in immune response and inflammation (15). In our studies, the specific inflammation-related pathways that were affected were different between the two long-term intervention studies. In Chapter 3, these were interferon signalling pathways in PBMCs, whereas in Chapter 4 these were complement pathways in adipose tissue. These findings indicate that the mechanisms that may responsible for the observed gene expression effects are also different. The mechanism by which the two longterm fatty acid interventions may have exerted their effects are discussed in their respective chapters in this thesis (Chapter 3 and Chapter 4). Briefly, regarding Chapter 3, one mouse study found that n-3 PUFA intake led to diminished interferon intracellular signalling after ex-vivo stimulation of immune cells with interferon- $\gamma$ (16). A similar mechanism could be present in our APOE4 carriers, though, to our knowledge, no studies exist that have examined the role of APOE genotype in this context. Many of the effects of N-3 PUFAs may be mediated by peroxisome proliferator-activated receptors (PPAR) (17). Of all fatty acids, N-3 PUFAs lead to the most pronounced PPAR activation (18). In context of this discussion, PPAR $\gamma$ is of particular interest, as it is expressed in adipose tissue and macrophages (19). 
Activation of PPAR $\gamma$ may cause anti-inflammatory changes in cells, through direct gene activation or via interfering with nuclear factor $\kappa \mathrm{B}(20)$. Possibly, the already increased levels of interferon signalling-related genes in APOE4 carriers makes them more susceptible to the anti-inflammatory effects of fish-oil supplementation, leading to decreased levels after supplementation.

The beneficial effects of medium-chain saturated fatty acids have so far been considered to mainly take place in the liver, especially post-prandially by an increase in energy expenditure due to rapid beta-oxidation of these fatty acids (21). However, our findings in Chapter 4 show that possibly some of the effects on adipose tissue are directly regulated by MC-SFAs, as some of these reach the adipose tissue (22). Interestingly, one study showed that mediumchain fatty acids can bind to and activate PPAR $\gamma$ (23). However, the amount of MC-SFAs reaching the adipose tissue is thought to be relatively minor (24), so it is questionable whether the effects that we observe are directly PPAR-induced or may be regulated through indirect mechanisms. In conclusion, the effects of fish-oil supplementation in APOE4 carriers may be a direct anti-inflammatory effect of these fatty acids, whereas the effect medium-chain saturated fatty acids on adipose tissue inflammatory gene expression may be directly regulated via PPAR $\gamma$ or indirectly regulated by other changes in the adipose tissue.

Since the adipose tissue as well as white blood cells in the circulation play a key role in obesityassociated metabolic disease, targeting both tissues with a combined medium-chain saturated fatty acid and fish-oil intervention may have additional anti-inflammatory effects. Especially persons who are at-risk of metabolic disease, such as APOE4-carriers, persons with obesity or those who already have the metabolic syndrome may benefit from such an intervention. Interestingly, in a small study (25), 10 healthy subjects were given a 14-day $1500 \mathrm{kcal}$ diet, administered in the form of a formula drink, containing $72 \%$ medium-chain fatty acids and $22 \%$ n-3 PUFA, or a control diet containing an iso-energetic amount of long-chain fatty acids, containing 33\% oleic acid, 30\% palmitic acid and 28\% linoleic acid. The medium-chain and PUFA group showed a decrease in plasma triglycerides and total cholesterol, whereas the control group showed a decrease in total cholesterol only. Even though this was a small and short-term study and the intervention contained large amounts of fatty acids, beneficial effects of a combined medium-chain and PUFA diet were observed. It would be very interesting to study the long-term effects of a medium-chain and PUFA diet in an intervention, which may be a combination of our interventions from Chapter 3 and Chapter 4, on other parameters, such as PBMC and adipose tissue gene expression as well as body composition and circulating 
inflammatory markers.

\section{Apolipoprotein E4}

\section{Direct transcriptional effects of APOE4}

Regarding APOE4, recently some interesting findings have been published that may be of interest to our already published findings in Chapter 3. A recent study showed that APOE can translocate to the nucleus of cells and bind with high affinity to DNA, acting as a transcription factor (26). APOE4-unique binding sites were found in the promotor region of $\sim 1700$ genes. Interferon- $\lambda$ was one of the genes identified as APOE4 target gene and was also among the top potential upstream regulators in our analysis. Potentially, increased IFN- $\lambda$ expression due to the APOE4 genotype may be responsible for the increase in IFN targets genes that we observed. The response to dietary fatty acids, especially to n-3 PUFAs has been shown to be partly dependent on APOE4 genotype, with APOE4 carriers showing larger decreases in plasma triglycerides upon supplementation with fish-oil compared to non-carriers (27). In Chapter 3, we observed APOE4-specific anti-inflammatory actions of fish-oil, which decreased expression of interferon signalling pathways. Besides triglyceride lowering, these findings point to additional anti-inflammatory actions of fish-oil in APOE4 carriers.

\section{Dual role of APOE4}

Many detrimental effects of APOE4 have been described, including chronic inflammation, increased risk of Alzheimer's disease, high blood lipids, lower life expectancy and increased risk of cardiovascular disease. Given these adverse effects, from an evolutionary viewpoint, this raises the question why the APOE4 allele has persisted until today. It is hypothesized that the increased inflammatory responses in APOE4 carriers may have a protective effect against viral and bacterial infections, especially in early stages of the life (28). APOE4 was shown to be beneficial during hepatitis $C$ virus infection by protecting against liver damage (29) and also to inhibit the growth of the malaria bacteria in red blood cells (30). Furthermore, it was shown to have protective effects on brain development in Brazilian children with a heavy diarrhea burden (31). Our findings may provide some insight into the mechanisms behind these observations, as type 1 interferons have been shown to be essential in the activation of anti-viral and anti-bacterial immune responses (32). From this, we hypothesize that increased expression of interferon signalling and interferon target genes may be beneficial for protecting 
against infectious diseases, but may be detrimental by increasing risk of cardiovascular and Alzheimer's disease.

\section{Post-prandial effects of dietary fatty acids}

In Chapter 5 and Chapter 6, we studied the gene expression responses in PBMCs and whole blood to acute high-fat loads. Consumption of these high-fat loads have been shown to lead to increased triglyceride levels in plasma for several hours post-prandially, whereas free fatty acids show an initial decrease in plasma levels ( 2 hours) and eventually rise above baseline (4 - 6 hours) $(33,34)$. Exposure of circulating white blood cells to this high-lipid environment, especially at 4 to 6 hours post-prandially, may be the cause of the gene expression changes that we observe. In Chapter 5, we studied changes in whole blood gene expression profiles, whereas in Chapter 6 we studied PBMC gene expression profiles. Initially, we set out to include the data of Chapter $\mathbf{5}$ in the meta-analysis of Chapter 6. However, we observed large differences in the gene expression response to the high-fat challenge separating this dataset from the other datasets. These differences may be the result of differences in cell populations between whole blood and PBMCs, or may be caused by differences in microarray platform between the Illumina HumanHT-12 V4 BeadArrays and the Affymetrix GeneChips. Even though Chapter 5 focussed on differences between groups (healthy subjects, healthy subjects after a high-fat high-calorie intervention and subjects with the metabolic syndrome), we did observe some postprandial changes in gene expression profiles that were similar to those observed in Chapter 6. In both chapters, we observed an upregulation of inflammationrelated pathways in response to a high-fat challenge, which may be the response to the increased stress associated with the high-lipid post-prandial environment and may be a way of the cells to cope with this stress. Interestingly, subjects with the metabolic syndrome do not show this inflammatory response (Chapter 5), which may reflect a diminished cellular response capacity.

Interestingly, the specific inflammation-related pathways that were upregulated in the studies differed. In the whole blood study, these were TLR cascade pathways, whereas in the PBMC studies these were interferon signalling pathways. Perhaps differences in cell types may be responsible for this. Whole blood consists for a large part of neutrophils, that are virtually absent in PBMCs which consist of lymphocytes and monocytes. TLRs are an important part of innate immunity, serving as pathogen recognition receptors (35) and have been shown to 
be essential for neutrophil activation (36). We hypothesize that the TLR signalling pathway may be relatively more active in neutrophils compared to monocytes and lymphocytes, leading to the differences in inflammation-related gene expression that we observe. Interestingly, TLR signalling has been shown to lead to activation of nuclear factor- $\kappa \mathrm{B}$, which subsequently induces expression of pro-inflammatory nuclear factor- $\kappa \mathrm{B}$-target genes [2022]. Since interferon $-\gamma$ is a nuclear factor- $\kappa B$-target gene [23], the increase in the expression of genes in the interferon signalling pathway, as observed in Chapter 6, may be instigated by this mechanism. Furthermore, a significant increase in the number as well as activation of neutrophils has been observed post-prandially (37-39). Taken together, it is possible that the postprandial pro-inflammatory response may be initiated in neutrophils, followed by inflammatory activation of other immune cells.

\section{Combining long-term fatty acid interventions with high-fat challenges}

Based on observations in Chapter 5, we speculated that subjects with the metabolic syndrome may show a blunted inflammation-related gene expression response compared to healthy subjects. Furthermore, after the 4-week of high-fat high-calorie diet, we observed a shift in post-prandial gene expression response of TLR pathways in healthy subjects towards a more MetS-like response, maybe suggesting that the subtle changes in metabolic status induced by a 4-week high-calorie diet may be observed in this pathway. This raises the question whether the inflammation-related gene expression response to a high-fat challenge can be used to examine changes in health status in other interventions, such as the fish-oil supplementation (Chapter 3) or medium-chain saturated fatty acid intervention (Chapter 4). Based on results from Chapter 5, we concluded that changes in fasting gene expression to the 4-week high-fat high-calorie diet are much more pronounced compared the changes in post-prandial high-fat challenge gene expression responses. Moreover, the combination of high-fat challenges with transcriptome analyses may provide some interesting findings, especially in inflammationrelated pathways. Nevertheless, fasting gene expression profiles appear more responsive to a 4-week high-fat high-calorie dietary intervention and may, therefore, be more suited for studying the effects of such an intervention. It should, however, be noted that Chapter $\mathbf{5}$ describes a small study with only a short dietary intervention period and it remains uncertain whether these findings can be confirmed in larger and longer studies and with different dietary interventions. 


\section{Issues with biological interpretation of microarray results}

Nutritional interventions induce only small changes or limited significant changes at the single-gene level. To prevent selection of false positive genes, we used several pathway analysis tools to select the pathways in which multiple genes are significantly differentially expressed. This increases the chance of finding potential biological relevant changes and decreases the chance of false discoveries. Gene set enrichment analysis is especially valuable, as it does not require $\mathrm{p}$-value and/or fold change cut-off values, but uses a continuous variable to rank genes (40). In this analysis method, gene sets (pathways) that are significantly positively or negatively enriched in the ranked gene list are considered differentially upregulated or downregulated respectively. A disadvantage of using pathway analyses is the introduction of bias, as we are reliant on pathway databases that are based on prior knowledge. Firstly, some pathways may not be active in the tissue that we study. Secondly, our analyses may be biased towards the most well-studied and described pathways, such as cell cycle and cancer-related pathways, hindering the potential for the generation of new hypotheses. We partly combatted the latter problem, by combining pathways from several pathway-databases in order to maximize the diversity of pathways that we analyse. Even though pathway analyses provide great value for finding potential biologically meaningful changes in gene expression profiles, one should not rely on pathways analyses only, as pathway databases are based on only a small part of all available literature. Therefore, it is important to also examine individual gene expression changes in differentially expressed pathways, and examine literature that may be valuable in the context of microarray study findings.

An issue with microarray analyses in most studies in this thesis (chapters 2, 3, 4 and 6), is that whole genome gene expression changes were not the primary outcome parameter in these studies. As a consequence, microarray analyses were performed as secondary retrospective analyses and the described analyses may therefore not have had an optimal study design. Performing studies with the specific goal of transcriptome analyses will increase power as a higher numbers of subjects can be measured and group size can be balanced. As an example, in Chapter 3, the original study consisted of 302 subjects, of which 96 were assigned to the $1800 \mathrm{mg} /$ day fish-oil supplementation group that we studied. Of these 96 subjects, microarray measurements were only available in 23. Performing microarrays in all subjects would have been very valuable for increasing the validity of our findings, as well as allow for additional analyses of other factors besides APOE genotype that may affect the response to fish-oil supplementation. 
Observations by others and us showed that between-subject variation in gene expression profiles is much larger than within-subject variation. From this thesis, it is clear that there are many factors that influence gene expression profiles and changes in these profiles. These findings highlight the importance of a study design, in which multiple interventions are performed within one subject; i.e a cross-over design, or, when this is not possibly to have large groups to analyse.

Transcription is only a small part of the total of cellular and organism biology, and regulation by food takes place at many more levels, including epigenetic, post-transcriptional and post-translational level. As a consequence, microarray studies are inherently hypothesisgenerating. To aid biological interpretation, microarray findings could be integrated with established biomarkers or data gathered using other -omics technologies.

\section{Potential of microarray meta-analysis}

With the increasing quantity of publicly available microarray datasets in repositories, such as GEO (41), ArrayExpress (42), and the Phenotype database (www.dbnp.org), several methods for microarray data integration have been developed and applied (43). Integration of data from separate studies provides opportunities for increasing reliability and generalizability of findings. In Chapter 6, we performed such a meta-analysis in the field of nutrigenomics. In this proof-of-principle study, we applied several meta-analysis methods to integrate data from 3 separate high-fat challenge studies. Using these methods, we identified several pathways that are consistently changed upon a high-fat challenge across studies from different labs. These findings provide evidence of a general response to a high-fat challenge that can be consistently measured, even in different labs, at different times, by different researchers, and using different microarray platforms. As discussed in Chapter 6, in part, this response may be the consequence of circadian oscillations in gene expression. However the gene expression response to a high-fat challenge, which contains large amounts of fat (50-100 gram) can be expected to be large, especially compared to the response to long-term dietary interventions which are, in general, milder interventions. In contrast to acute high-fat challenges, long-term interventions may cause changes in functioning of different metabolic organs, such intestine, liver or adipose tissue, resulting in alteration in secretion of signalling molecules such as adipokines or hepatokines, leading to systemic changes in the blood that can be measured in circulating blood cells. Furthermore, for long-term interventions ranging from weeks to 
months, the relative influence of other phenotypic, genotypic and environmental factors may be larger compared to that of acute high fat challenges, as these factors also influence systemic metabolic status. As a result, it can be expected that changes in gene expression to long-term interventions are smaller and more variable between subjects (44).

In summary, long-term changes in gene expression to a dietary intervention may reflect systemic changes in the body, whereas gene expression response to a challenge test may be very direct responses designed to deal with the post-prandial environment. It remains to be elucidated whether meta-analyses can be applied to examine the effect of these longterm dietary interventions. One area of research, in which several long-term intervention studies are available and meta-analysis may be applied is that of fish-oil supplementation. To our knowledge, at least six studies have been performed that examined the effects of fishoil supplementation on PBMC or whole blood gene expression using microarrays $(3,6,7$, 45-47). Many differences exist between studies, such as study population, duration, fish oil supplement composition and microarray platform. Nevertheless, performing a meta-analysis of these studies may provide valuable insight to both the applicability of these methods in long-term nutritional intervention studies as well as the identification of general molecular mechanisms underlying the effects of fish-oil.

\section{The future of nutrigenomics}

The research presented in this thesis falls in the field of nutrigenomics, which focusses on the mechanisms by which nutrition affects our cells and body at different levels, including the transcriptome, proteome and the metabolome. Increasing our understanding of how nutrients influence cellular and organism metabolism may allow for effective dietary-intervention strategies for the prevention of diet-related diseases (48). Even though many discoveries on the effects of nutrients on transcriptome, proteome and metabolome have been made, the interplay between genotype, phenotype, diet, other environmental factors and development of disease is extremely complex. Due to this large complexity, at present, nutrigenomics-based dietary advice on the basis of phenotypic or genotypic characteristics is not applicable in the general population. Nevertheless, transcriptome analysis have shown differences in dietary response between individuals (Chapter 2 and Chapter 3) though many questions remain to be answered. As an example, even though sex is universally recognized as an important determinant of dietary response, relatively few studies have actually examined the impact 
of sex on response to diet. In Chapter 2, the two studies that studied sex, only did so in a retrospective analysis. Specifically designed studies focusing on the interaction between sex and diet are highly needed, as most studies in the past have focused only on males. A similar story goes for other phenotypic and genotypic factors. For example, we showed inflammationinhibiting effects on gene expression profiles in PBMCs induced by fish-oil supplementation in APOE4 carriers. Even though these changes may be beneficial, they do not provide sufficient evidence for dietary recommendations. Ultimately, integration of multi-omics data may be the key to personalized nutrition. In 2015, Zeevi et al. (49) performed a landmark study in which they devised a machine-learning algorithm that integrated blood parameters, dietary habits, anthropometrics, physical activity, and gut microbiota. Using this algorithm, they were able to predict postprandial glycemic responses to real-life meals. The algorithm was validated in an independent cohort and finally shown in a personalized nutrition intervention study to result in lower post-prandial glycemic responses. In the future, similar techniques for personalized prediction of nutritional effects may be applied to other dietary interventions or outcome measures. Such prediction algorithms may be used, for example, for the identification of individuals who may profit the most from a fish-oil intervention, or be used to identify which diet may be optimal for individuals who carry the APOE4 allele or have characteristics of the metabolic syndrome. Developing these prediction algorithms will require large amounts of data. In this regard, being able to combine data from different studies, as touched upon in Chapter 6 will be very valuable. Ultimately, all these efforts may pave the way for personalized nutrition for the prevention and treatment of metabolic disease.

\section{Conclusion}

In this thesis, we studied the molecular mechanisms underlying the response to dietary interventions with different types of fatty acids. We observed anti-inflammatory actions of two long-term interventions. Fish-oil supplementation induced these anti-inflammatory changes in PBMC gene expression profiles in APOE4 carriers, whereas medium-chain saturated fatty acids induced these in the adipose tissue of overweight individuals.

High-fat challenges induce pro-inflammatory gene expression profiles in whole blood and PBMCs, possibly by direct actions of fatty acids. We showed that these changes were diminished in subjects with the metabolic syndrome, suggesting a state of decreased cellular flexibility. Lastly, by performing a meta-analysis of PBMC microarray data from acute 
nutritional challenge studies, we showed that the gene expression response was consistent across different studies.

\section{References}

1. Esser D, van Dijk SJ, Oosterink E, Lopez S, Muller M, Afman LA. High fat challenges with different fatty acids affect distinct atherogenic gene expression pathways in immune cells from lean and obese subjects. Mol Nutr Food Res. 2015;59(8):1563-72.

2. Matone A, O'Grada CM, Dillon ET, Morris C, Ryan MF, Walsh M, et al. Body mass index mediates inflammatory response to acute dietary challenges. Mol Nutr Food Res. 2015;59(11):2279-92.

3. Rudkowska I, Paradis AM, Thifault E, Julien P, Tchernof A, Couture P, et al. Transcriptomic and metabolomic signatures of an n-3 polyunsaturated fatty acids supplementation in a normolipidemic/normocholesterolemic Caucasian population. The Journal of nutritional biochemistry. 2013;24(1):54-61.

4. van Erk MJ, Pasman WJ, Wortelboer HM, van Ommen B, Hendriks HF. Short-term fatty acid intervention elicits differential gene expression responses in adipose tissue from lean and overweight men. Genes Nutr. 2008;3(3-4):127-37.

5. Radonjic M, van Erk MJ, Pasman WJ, Wortelboer HM, Hendriks HF, van Ommen B. Effect of body fat distribution on the transcription response to dietary fat interventions. Genes Nutr. 2009;4(2):143-9.

6. Schmidt S, Stahl F, Mutz KO, Scheper T, Hahn A, Schuchardt JP. Different gene expression profiles in normoand dyslipidemic men after fish oil supplementation: results from a randomized controlled trial. Lipids in health and disease. 2012;11:105.

7. Bouwens M, van de Rest O, Dellschaft N, Bromhaar MG, de Groot LC, Geleijnse JM, et al. Fish-oil supplementation induces antiinflammatory gene expression profiles in human blood mononuclear cells. The American journal of clinical nutrition. 2009;90(2):415-24.

8. Mathis D, Shoelson SE. Immunometabolism: an emerging frontier. Nat Rev Immunol. 2011;11(2):81.

9. Lumeng CN, Bodzin JL, Saltiel AR. Obesity induces a phenotypic switch in adipose tissue macrophage polarization. J Clin Invest. 2007;117(1):175-84.

10. O’Neill LA, Kishton RJ, Rathmell J. A guide to immunometabolism for immunologists. Nat Rev Immunol. 2016;16(9):553-65.

11. Ouchi N, Parker JL, Lugus JJ, Walsh K. Adipokines in inflammation and metabolic disease. Nat Rev Immunol. 2011;11(2):85-97.

12. Dicker D, Salook MA, Marcoviciu D, Djaldetti M, Bessler H. Role of peripheral blood mononuclear cells in the predisposition of obese individuals to inflammation and infection. Obesity facts. 2013;6(2):146-51.

13. Sirota P, Hadi E, Djaldetti M, Bessler H. Difference in inflammatory cytokine production by mononuclear cells from obese and non-obese schizophrenic patients. Acta psychiatrica Scandinavica. 2015;132(4):301-5.

14. McLaren JE, Ramji DP. Interferon gamma: a master regulator of atherosclerosis. Cytokine \& growth factor reviews. 2009;20(2):125-35.

15. O'Grada CM, Morine MJ, Morris C, Ryan M, Dillon ET, Walsh M, et al. PBMCs reflect the immune component of the WAT transcriptome--implications as biomarkers of metabolic health in the postprandial state. Mol Nutr Food Res. 2014;58(4):808-20.

16. Irons R, Fritsche KL. Omega-3 polyunsaturated fatty acids impair in vivo interferon- gamma responsiveness via diminished receptor signaling. The Journal of infectious diseases. 2005;191(3):481-6.

17. Georgiadi A, Kersten S. Mechanisms of gene regulation by fatty acids. Advances in nutrition. 2012;3(2):127-34. 
18. Kliewer SA, Sundseth SS, Jones SA, Brown PJ, Wisely GB, Koble CS, et al. Fatty acids and eicosanoids regulate gene expression through direct interactions with peroxisome proliferator-activated receptors alpha and gamma. Proceedings of the National Academy of Sciences of the United States of America. 1997;94(9):4318-23.

19. Bookout AL, Jeong Y, Downes M, Yu RT, Evans RM, Mangelsdorf DJ. Anatomical profiling of nuclear receptor expression reveals a hierarchical transcriptional network. Cell. 2006;126(4):789-99.

20. Calder PC. Omega-3 fatty acids and inflammatory processes. Nutrients. 2010;2(3):355-74.

21. Marten B, Pfeuffer M, Schrezenmeir J. Medium-chain triglycerides. International Dairy Journal. 2006;16(11):1374-82.

22. Sarda P, Lepage G, Roy CC, Chessex P. Storage of medium-chain triglycerides in adipose tissue of orally fed infants. The American journal of clinical nutrition. 1987;45(2):399-405.

23. Liberato MV, Nascimento AS, Ayers SD, Lin JZ, Cvoro A, Silveira RL, et al. Medium chain fatty acids are selective peroxisome proliferator activated receptor (PPAR) gamma activators and pan-PPAR partial agonists. PloS one. 2012;7(5):e36297.

24. Schonfeld P, Wojtczak L. Short- and medium-chain fatty acids in energy metabolism: the cellular perspective. Journal of lipid research. 2016;57(6):943-54.

25. Beermann C, Jelinek J, Reinecker T, Hauenschild A, Boehm G, Klor HU. Short term effects of dietary mediumchain fatty acids and n-3 long-chain polyunsaturated fatty acids on the fat metabolism of healthy volunteers. Lipids in health and disease. 2003;2:10.

26. Theendakara V, Peters-Libeu CA, Spilman P, Poksay KS, Bredesen DE, Rao RV. Direct Transcriptional Effects of Apolipoprotein E. The Journal of neuroscience : the official journal of the Society for Neuroscience. 2016;36(3):685-700.

27. Caslake MJ, Miles EA, Kofler BM, Lietz G, Curtis P, Armah CK, et al. Effect of sex and genotype on cardiovascular biomarker response to fish oils: the FINGEN Study. The American journal of clinical nutrition. 2008;88(3):61829.

28. Finch CE. Evolution in health and medicine Sackler colloquium: Evolution of the human lifespan and diseases of aging: roles of infection, inflammation, and nutrition. Proceedings of the National Academy of Sciences of the United States of America. 2010;107 Suppl 1:1718-24.

29. Wozniak MA, Itzhaki RF, Faragher EB, James MW, Ryder SD, Irving WL, et al. Apolipoprotein E-epsilon 4 protects against severe liver disease caused by hepatitis C virus. Hepatology. 2002;36(2):456-63.

30. Fujioka H, Phelix CF, Friedland RP, Zhu X, Perry EA, Castellani RJ, et al. Apolipoprotein E4 prevents growth of malaria at the intraerythrocyte stage: implications for differences in racial susceptibility to Alzheimer's disease. Journal of health care for the poor and underserved. 2013;24(4 Suppl):70-8.

31. Oria RB, Patrick PD, Zhang H, Lorntz B, de Castro Costa CM, Brito GA, et al. APOE4 protects the cognitive development in children with heavy diarrhea burdens in Northeast Brazil. Pediatric research. 2005;57(2):310-6.

32. Perry AK, Chen G, Zheng D, Tang H, Cheng G. The host type I interferon response to viral and bacterial infections. Cell Res. 2005;15(6):407-22.

33. Bouwens M, Grootte Bromhaar M, Jansen J, Muller M, Afman LA. Postprandial dietary lipid-specific effects on human peripheral blood mononuclear cell gene expression profiles. The American journal of clinical nutrition. 2010;91(1):208-17.

34. Esser D, Oosterink E, op 't Roodt J, Henry RM, Stehouwer CD, Muller M, et al. Vascular and inflammatory high fat meal responses in young healthy men; a discriminative role of IL-8 observed in a randomized trial. PloS one. 2013;8(2):e53474.

35. Akira S, Uematsu S, Takeuchi O. Pathogen recognition and innate immunity. Cell. 2006;124(4):783-801.

36. Prince LR, Whyte MK, Sabroe I, Parker LC. The role of TLRs in neutrophil activation. Curr Opin Pharmacol. 2011;11(4):397-403. 
37. Kardinaal AF, van Erk MJ, Dutman AE, Stroeve JH, van de Steeg E, Bijlsma S, et al. Quantifying phenotypic flexibility as the response to a high-fat challenge test in different states of metabolic health. FASEB J. 2015;29(11):4600-13.

38. van Oostrom AJ, Sijmonsma TP, Verseyden C, Jansen EH, de Koning EJ, Rabelink TJ, et al. Postprandial recruitment of neutrophils may contribute to endothelial dysfunction. Journal of lipid research. 2003;44(3):57683.

39. Esser D, van Dijk SJ, Oosterink E, Muller M, Afman LA. A high-fat SFA, MUFA, or n3 PUFA challenge affects the vascular response and initiates an activated state of cellular adherence in lean and obese middle-aged men. The Journal of nutrition. 2013;143(6):843-51.

40. Subramanian A, Tamayo P, Mootha VK, Mukherjee S, Ebert BL, Gillette MA, et al. Gene set enrichment analysis: a knowledge-based approach for interpreting genome-wide expression profiles. Proceedings of the National Academy of Sciences of the United States of America. 2005;102(43):15545-50.

41. Edgar R, Domrachev M, Lash AE. Gene Expression Omnibus: NCBI gene expression and hybridization array data repository. Nucleic Acids Res. 2002;30(1):207-10.

42. Kolesnikov N, Hastings E, Keays M, Melnichuk O, Tang YA, Williams E, et al. ArrayExpress update--simplifying data submissions. Nucleic Acids Res. 2015;43(Database issue):D1113-6.

43. Walsh CJ, Hu P, Batt J, Santos CC. Microarray Meta-Analysis and Cross-Platform Normalization: Integrative Genomics for Robust Biomarker Discovery. Microarrays. 2015;4(3):389-406.

44. Afman LA, Muller M. Human nutrigenomics of gene regulation by dietary fatty acids. Progress in lipid research. 2012;51(1):63-70.

45. Myhrstad MC, Ulven SM, Gunther CC, Ottestad I, Holden M, Ryeng E, et al. Fish oil supplementation induces expression of genes related to cell cycle, endoplasmic reticulum stress and apoptosis in peripheral blood mononuclear cells: a transcriptomic approach. J Intern Med. 2014;276(5):498-511.

46. Vedin I, Cederholm T, Freund-Levi Y, Basun H, Garlind A, Irving GF, et al. Effects of DHA-rich n-3 fatty acid supplementation on gene expression in blood mononuclear leukocytes: the OmegAD study. PloS one. 2012;7(4):e35425.

47. Gorjao R, Verlengia R, Lima TM, Soriano FG, Boaventura MF, Kanunfre CC, et al. Effect of docosahexaenoic acid-rich fish oil supplementation on human leukocyte function. Clin Nutr. 2006;25(6):923-38.

48. Muller M, Kersten S. Nutrigenomics: goals and strategies. Nat Rev Genet. 2003;4(4):315-22.

49. Zeevi D, Korem T, Zmora N, Israeli D, Rothschild D, Weinberger A, et al. Personalized Nutrition by Prediction of Glycemic Responses. Cell. 2015;163(5):1079-94. 


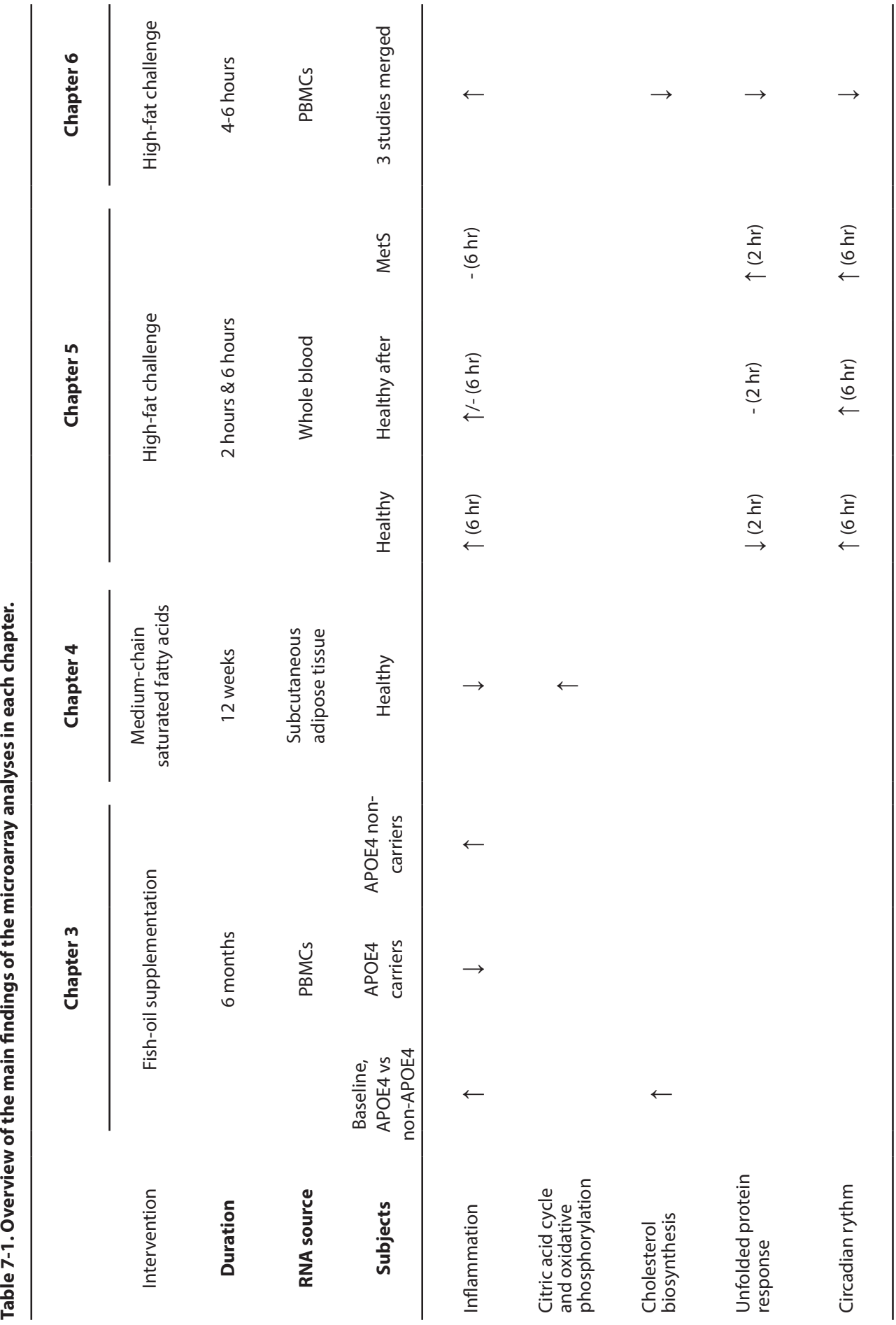


Summary 
Various types of dietary fatty acids have different effects on human health. The aim of this thesis was to increase our understanding of the molecular mechanisms underlying the effects of dietary fatty acids. To do this, we examined changes in whole genome gene expression profiles upon both acute as well as longer term dietary fatty acid interventions. Furthermore, from previous research, it is clear that large inter-individual differences in the response to dietary fatty acids exist. We used whole genome gene expression analyses to increase our understanding of the mechanisms underlying some of these inter-individual differences.

Many modifiable and non-modifiable factors can be the cause of these inter-individual differences. In Chapter 2, we reviewed all studies that examined differences in the transcriptional response to dietary interventions based on the presence of one of these factors. These include gender, age, BMI, body composition, blood lipid levels and gut microbial composition. We conclude that transcriptome analyses are well-suited for studying the underlying mechanisms behind these differences in the response to diet. Nevertheless, the number of studies that use this approach remains limited.

Another factor that may modify the response to a dietary intervention is genetics, e.g. the apolipoprotein E4 (APOE4) variant. People who carry the APOE4 allele have an increased risk of cardiovascular disease. Fish-oil supplementation may help in the prevention of cardiovascular disease, though inter-individual differences in the response to n-3 polyunsaturated fatty acids on gene expression profiles have been observed. In Chapter 3, we aimed to assess the impact of APOE4 on peripheral blood mononuclear cell (PBMC) whole genome gene expression at baseline and following a 6-month fish-oil intervention. We observed increased gene expression of IFN signaling and cholesterol biosynthesis pathways in APOE4 carriers, which might explain part of the association between APOE4 and CVD. Furthermore, fish-oil supplementation may be beneficial by decreasing interferon signallingrelated gene expression in APOE4 carriers.

Another long-term dietary intervention with fatty acids was studied in Chapter 4. We examined the effect of a 12-week high medium-chain saturated fatty acid diet on subcutaneous adipose tissue gene expression profiles. We observed increased expression of genes involved in oxidative energy metabolism and decreased inflammation-related gene expression due to the high medium-chain saturated fatty acid intake. Considering the role of the adipose tissue in sustaining the low-grade inflammation that is associated with obesity, these findings may be indicative of a more anti-inflammatory phenotype of the adipose tissue. We concluded that medium-chain saturated fatty acids may potentially have beneficial effects on adipose tissue 
functioning.

Besides studying the effects of long-term interventions with fatty acids on whole genome gene expression, we also examined the effects of acute high-fat challenges. In Chapter 5, we determined the additional value of determining whole genome gene expression changes in response to a high-fat challenge compared to assessment at fasting only. In addition, we aimed to identify whether a 4 week high-fat high-calorie diet can induce a shift in gene expression profiles in healthy subjects towards a metabolic syndrome-like gene expression profile. We found that fasting whole blood whole genome gene expression profiles are highly responsive to a 4-week high-fat high-calorie diet, with changes in in the direction of a metabolic syndrome-like gene expression profile. High-fat challenge responses in healthy subjects show only minimal changes in gene expression upon the dietary intervention and a marginal shift in the direction of the metabolic syndrome. We concluded that fasting gene expression profiles are more responsive compared to high-fat challenge responses to a 4-week high-fat high-calorie diet.

Besides Chapter 5, several other studies have also examined changes in whole genome gene expression in blood cells induced by high-fat challenges. In Chapter 6, we combined microarray data from four high-fat challenge studies varying in study population, challenge composition and research laboratory. By performing this meta-analysis, we showed a general PBMC whole genome gene expression response to a high-fat challenge. We concluded that a meta-analysis provides added value for the discovery of consistently differentially expressed genes and pathways compared to selecting only those genes and pathways that are identified in all separate studies.

In conclusion, in this thesis we showed differences in the whole genome gene expression response to fish-oil supplementation in PBMCs of APOE4 carriers vs non-carriers. Furthermore, the effects on whole genome gene expression of the two long-term dietary interventions, i.e. the fish-oil supplementation in PBMCs of APOE4 carriers and the high medium-chain saturated fatty acid diet in adipose tissue, may be beneficial by downregulation of gene expression related to inflammation. We also showed that whole genome gene expression responses to high-fat challenges are affected by a 4 -week high-fat high-calorie diet, though changes in fasting gene expression profiles are much more pronounced. Finally, we showed the value of meta-analysis of microarray data in high-fat challenge studies for identifying the general response to a high-fat challenge. 
Acknowledgements - Dankwoord 
Na meer dan 4 jaar werken is dit boekje dan eindelijk af. Het zijn voor mij zowel wetenschappelijk als persoonlijk heel leerzame jaren geweest. In dit dankwoord wil ik graag iedereen bedanken die heeft bijgedragen aan de voltooiing van dit proefschrift.

Lydia, als co-promotor en directe begeleider ben jij de persoon die het meest heeft bijgedragen aan de voltooiing van mijn proefschrift. Bedankt voor het vertrouwen dat je gaf door mij aan te nemen voor dit project. Ik heb heel veel van je geleerd als wetenschapper en ik heb je enthousiasme en directe manier van communiceren altijd zeer gewaardeerd.

Jildau, als tweede co-promotor heb je een grote bijdrage geleverd aan meerdere hoofdstukken uit mijn proefschrift. Bedankt voor je wetenschappelijke input en fijne, pragmatische manier van werken. Onze discussies bij TNO over de papers heb ik altijd als heel prettig ervaren en zijn van grote waarde geweest. Michael, bedankt voor het vertrouwen dat je gaf door mij aan te nemen voor dit project. In de beginjaren van mijn promotie hadden we regelmatig discussies. Jouw brede visie heb ik altijd zeer gewaardeerd. Sander, bedankt dat ik deel kon uitmaken van jouw groep.

Graag wil ik de leden van de promotiecommissie Edith Feskens, Peter van Baarlen, Chris Evelo en Hanno Pijl bedanken voor het lezen van mijn proefschrift en het voeren van de oppositie.

Marijana, you were my supervisor at TNO at the beginning of my $\mathrm{PhD}$. Even though this time was short, I learned a lot from you. Mensen van de systeembiologie groep bij TNO, bedankt voor alle hulp. I would especially like to thank the other PhD-students at TNO Sultan, Yu, Dulce and Abeer. It was nice to share this experience with you. Thank you for making my occasional days at TNO so much fun.

Het grootste deel van mijn promotieperiode vond plaats in kamer 0051 van de Valk. Diederik, als sfeermaker was jij heel belangrijk in de kamer. Bedankt voor de fijne tijd. Ik ben blij dat ik jou als paranimf mag hebben. Rinke, ik ken jou als een excellente en gedreven wetenschapper en jij bent daarom een voorbeeld voor mij. Sophie, bedankt voor de interesse die je toonde in mijn onderzoek en het tegengewicht dat je gaf ten opzichte van de twee andere mannen in de kamer. Ook mijn andere kamergenoten in de Valk Danielle, Milene en Montserrat, thank you! Na de verhuizing heb ik de laatste maanden van mijn promotie doorgebracht in Helix met een aantal nieuwe kamergenoten. Roland, Philip, Mara en Merel, bedankt voor het delen van de laatste loodjes en succes met jullie eigen onderzoek.

Wilma, Jocelijn, Klaske, Mark en Marco, bedankt voor jullie interesse in mijn onderzoek 
en opmerkingen en vragen bij de donderdagochtendpresentaties. Guido, je hebt me vaak geholpen met microarray analyses en R. Ik ben blij dat je mijn paranimf wil zijn.

Mechteld, bedankt voor alle hulp bij het labwerk en dat ik altijd met vragen bij jou terecht kon. Daarnaast ook bedankt voor het begeleiden van de (lastige) studenten. Inge, onze promotietrajecten zijn ongeveer gelijktijdig verlopen. Ik vond het fijn dat ik af en toe met vragen terecht kon en bedankt voor je input tijdens besprekingen. Charlotte, bedankt voor alle hulp onder andere bij Lydia-groep meetings en paper-besprekingen. Philip, in het begin heb je veel geholpen bij de microarray analyses. Bedankt voor al je inspanningen. Parastoo, we started and finished our $\mathrm{PhD}$ at the same time and both worked on bioinformatics. It was nice to be able to share these experiences. Good luck in your new job in Germany! Ya, you will defend your thesis shortly after me. It was nice that we could discuss our common problems in the months before our defences. Good luck defending your thesis! Neeraj, thanks for the nice talks in the hallway.

Leden van PhD-tour commissie, de organisatie en uiteindelijke reis waren een groot succes. Bedankt voor de fijne tijd!

Other PhD-students of the NMG and pharma groups Antwi, Rogier, Aafke, Lily, Wieneke, Suzanne, Paulien, Jvalini, Katja, Fenni and Frits, thanks for the fun times. Xanthe, Benthe, Miranda, jullie zijn pas net begonnen met jullie promoties. Succes in de komende jaren! Postdocs Michiel and Nikkie, bedankt en succes in jullie verdere carrière. Karin, Carolien, Shohreh, Mieke, en Jenny, bedankt voor jullie hulp in het lab.

Kim, Arian, Niek, Rosanne, Boke en Rick. Inmiddels wonen we niet meer met zijn allen aan de Beeklustweg. Ik vind het fijn dat we elkaar zo regelmatig zien. Bedankt voor de fijne avonden en weekenden die we samen hebben doorgebracht.

Papa en mama, en zusters Lisanne en Ilse, bedankt dat ik altijd op jullie steun kon rekenen. Lisanne veel geluk met Mustafa en de kleine Emre. Ilse en Kevin veel geluk met elkaar in jullie nieuwe huis. Schoonfamilie Sylvia, Paula, Michel, Chayenna en Mitch, bedankt voor de gezelligheid en jullie interesse.

Lieve Sandra, toen ik begon met promoveren had ik jou nog niet ontmoet. Inmiddels zijn we al bijna vier jaar samen en kan ik me geen leven zonder jou meer voorstellen. Bedankt voor alles! 
About the author 


\section{Curriculum Vitae}

Juri Matualatupauw was born on March 31, 1985 in Apeldoorn, the Netherlands. He completed secondary school at the Veluws College in Apeldoorn. Thereafter, he started his bachelor in biomedical sciences at the Radboud University in Nijmegen, the Netherlands. Afterwards, Juri continued with the master program in Biomedical Sciences at the Radboud University. His did his first master thesis at the department of Biomaterials at the Radboud University Nijmegen Medical Center, where he investigated the effect of enamel matrix derivative on human primary bone cells. He then performed his second master thesis at the department of Physiology at the Radboud University Nijmegen Medical Center, where he investigated the changes in endothelial function and arterial remodeling in response to exercise training in patients with type 2 diabetes mellitus. Shortly after receiving his MSc degree in Biomedical Sciences, Juri started his $\mathrm{PhD}$ project 'Differences in transcriptional responses to acute and chronic dietary interventions with fatty acids in humans' at the Nutrition, Metabolism and Genomics group at Wageningen University in cooperation with the Netherlands Organisation for Applied Scientific Research TNO in Zeist, the Netherlands. He was supervised by copromotors Dr. Lydia Afman and Dr. Jildau Bouwman and promotor Prof. Dr. Sander Kersten. After completion of his PhD, Juri started working as a postdoc at the Host-Microbe Interactomics group at Wageningen University. 


\section{List of publications}

Matualatupauw JC, Roche HM, Bouwman J, Afman LA. Meta-analysis of high-fat challengeinduced changes in blood cell whole genome gene expression. In preparation

Matualatupauw JC, Afman LA, Bouwman J. Added value of using high-fat challenges to determine a high-fat high-calorie diet-induced shift towards the metabolic syndrome as measured in blood cell transcriptome. In preparation

Esser D, Geleijnse JM, Matualatupauw JC, Dower JI, Kromhout D, Hollman PCH, Afman LA. Pure flavonoid epicatechin and whole genome gene expression profiles in circulating immune cells in adults with elevated blood pressure. Submitted

Matualatupauw JC, Bohl M, Gregerson S, Hermansen K, Afman LA. Dietary medium-chain saturated fatty acids induce gene expression of energy metabolism-related pathways in adipose tissue of abdominally obese subjects. Int J Obes (Lond). In press

Matualatupauw JC, Afman LA. The use of transcriptomics as a tool to identify differences in the response to diet. In Kussmann M and Stover PJ (Eds.), Nutrigenomics and Proteomics in Health and Disease: Towards a Systems-Level Understanding of Gene-Diet Interactions (pp.1-18). Chichester, UK: John Wiley \& Sons.

Matualatupauw JC, Radonjic M, van de Rest O, de Groot LC, Geleijnse JM, Müller M, Afman LA. Apolipoprotein E genotype status affects habitual human blood mononuclear cell gene expression and its response to fish oil intervention. Mol Nutr Food Res. 2016 Jul;60(7):1649-60. 


\section{Overview of completed training activities}

\section{Discipline specific activities}

- NutriScience (Wageningen, the Netherlands, 2013)

- $10^{\text {th }} \mathrm{NuGO}$ week (Freising, Germany, 2013)

- Nutritional Science days (Deurne, the Netherlands, 2013)

- Nutrigenomics studies in humans (Castellammare di Stabia, Italy, 2014)

- $\quad 11^{\text {th }} \mathrm{NuGO}$ week (Castellammare di Stabia, Italy, 2014)

- Nutritional Science days (Deurne, the Netherlands, 2014)

- DairyHealth Symposium (Nibe, Denmark, 2015)

- Molecular Nutrition \& Regulation of Cardiometabolic Health (Dublin, Ireland, 2015)

- $\quad 12^{\text {th }}$ NuGOweek (Barcelone, Spain, 2015)

- Breakthroughs in Understanding Central and Peripheral Pathways in Obesity (New York, United States, 2015)

- DairyHealth Symposium (Skagen, Denmark, 2015)

\section{General activities}

- VLAG PhD week (Baarlo, the Netherland, 2012)

- $\quad$ Kick start R (Amsterdam, the Netherlands, 2013)

- Analysis of microarray and RNA Seq expression data using R/BioC and web tools (Rotterdam, the Netherlands, 2013)

- $\quad$ Project and time management (Wageningen, the Netherlands, 2015)

- $\quad$ Carreer guidance (Wageningen, the Netherlands, 2016)

\section{Optionals}

- Preparation research proposal (2012)

- Weekly scientific meetings NMG-pharma (2012-2016)

- Human Nutrition PhD tour United States (2015) 


\section{Colofon}

The research described in this thesis was financially supported by TNO and DairyHealth, a program financed by the The Danish Council for Strategic Research (no. 0603-004193), Arla Foods Ingredients Group P/S and the Danish Dairy Research Foundation.

Financial support from Wageningen University for printing this thesis is gratefully acknowledged.

Design and layout: Juri C. Matualatupauw

Printed by: Digiforce - ProefschriftMaken.nl

(C) Juri C. Matualatupauw, 2017 